\title{
Recent advances in organic luminescent materials with narrowband emission
}

\author{
Jung Min Ha${ }^{1}$, Seon Hyoung Hur ${ }^{1}$, Ambika Pathak', Ji-Eun Jeong (10 ${ }^{1,2}$ and Han Young Woo (D)
}

\begin{abstract}
The International Telecommunication Union announced a new color gamut standard of broadcast service television (BT 2020) for ultra-high-definition TV in 2012. To satisfy the wide-color gamut standard of BT 2020, monochromatic red $(R)$, green $(G)$, and blue $(B)$ emissions require a small full width at half-maximum, which is an important property for improving color purity. Although organic light-emitting diode (OLED) displays are currently one of the main types of display technologies, their broad emission via strong vibronic coupling between ground and excited states is a major hurdle to overcome in the development of next-generation wide-color gamut displays. Thus, the development of OLED emitters with narrowband R-G-B emissions is of great significance. In this review, the recent progress in the development of OLED materials with narrowband emission is summarized by grouping them into fluorescent, phosphorescent, and thermally activated delayed fluorescent emitters to reveal the correlation between molecular structures, optical properties, and device characteristics. We discuss rational molecular design strategies to achieve narrow photoluminescence and electroluminescence and the underlying mechanisms for controlling the emission bandwidth. Finally, the challenges in the realization of wide-color gamut OLED displays and the future prospects of such devices are discussed.
\end{abstract}

\section{Introduction}

Since the first organic light-emitting diode (OLED) was successfully demonstrated by Tang and Slyke in $1987^{1}$, OLEDs have been extensively studied in both academia and industry, becoming a mainstream display technology in fullcolor televisions and smartphones. They have various advantages, such as a light weight, fast response time, wide viewing angle, facile chemical tunability of emitting molecules, low energy consumption, compatibility with flexible plastic substrates, and form factors for various types of displays $^{2}$. Based on the light-emitting mechanisms, different types of OLEDs have been developed: fluorescence (1st generation)-based, phosphorescence (2nd generation)-

\footnotetext{
Correspondence: Ji-Eun Jeong (jieunj@krict.re.kr) or

Han Young Woo (hywoo@korea.ac.kr)

'Department of Chemistry, KU-KIST Graduate School of Converging Science and Technology, Korea University, Anam-ro 145, Seoul 02841, Republic of Korea

${ }^{2}$ Center for Advanced Specialty Chemicals, Korea Research Institute of Chemical Technology (KRICT), Jongga-ro 45, Jung-gu, Ulsan 44412, Republic of Korea
}

based, and thermally activated delayed fluorescence (TADF, 3rd generation)-based OLEDs ${ }^{3}$. Recently, extensive research on the 4th generation of OLEDs is in progress to improve the device efficiency, lifetime, and color purity in particular. Despite the many advantages of OLEDs, their emission spectra often show broad bandwidths, which are extremely detrimental to achieving high color purity for future highend display electronics such as high-definition TV and ultra-high-definition TV (UHDTV).

The "CIE (International Commission on Illumination) 1931 color space" was first defined based on tristimulus values; then, the modified CIE 1976 was announced, and both CIE 1931 and 1976 became the most widely accepted standards to define emission colors in the field of displays ${ }^{4}$. The CIE coordinate visualizes the entire range of colors that can be obtained by mixing the three primary colors (red (R), green (G), and blue (B)) by varying the wavelength and emission intensity. In 2012, the International Telecommunication Union (ITU) announced a new color gamut standard for UHDTVs called the Broadcast 

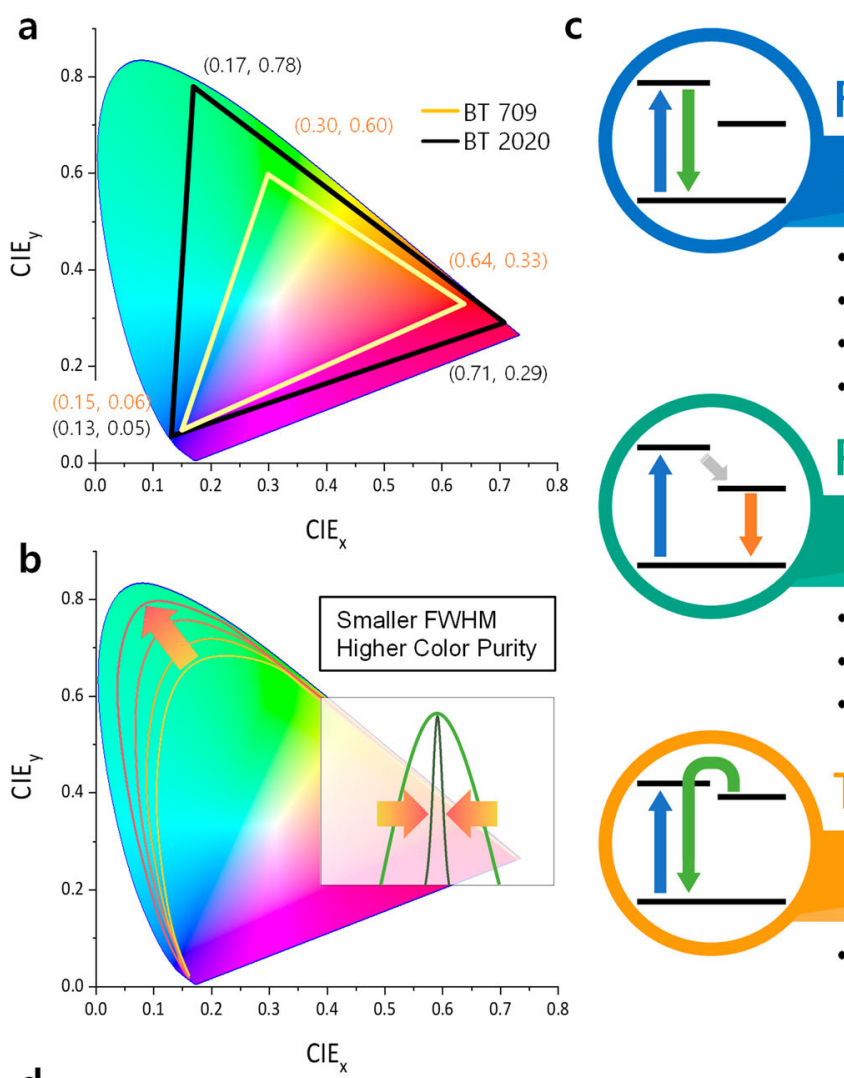

Fluorescence

Avoid charge transfer interaction

and intermolecular aggregation

- Twisted structures

- Planar and fused compounds

- Quinacridone derivatives

- BODIPY derivatives

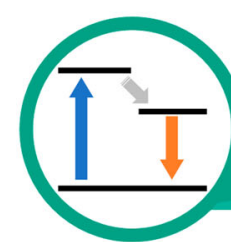

Phosphorescence

Increase of LC character

in MLCT/LC intermixed triplet states

- Octahedral iridium complexes with rigid ligands

- Square-planar platinum/palladium complexes

- Trivalent lanthanide complexes

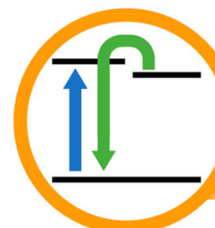

TADF

Multiple resonance effect

- B/N-based structure

: HOMO/LUMO separation on different atoms

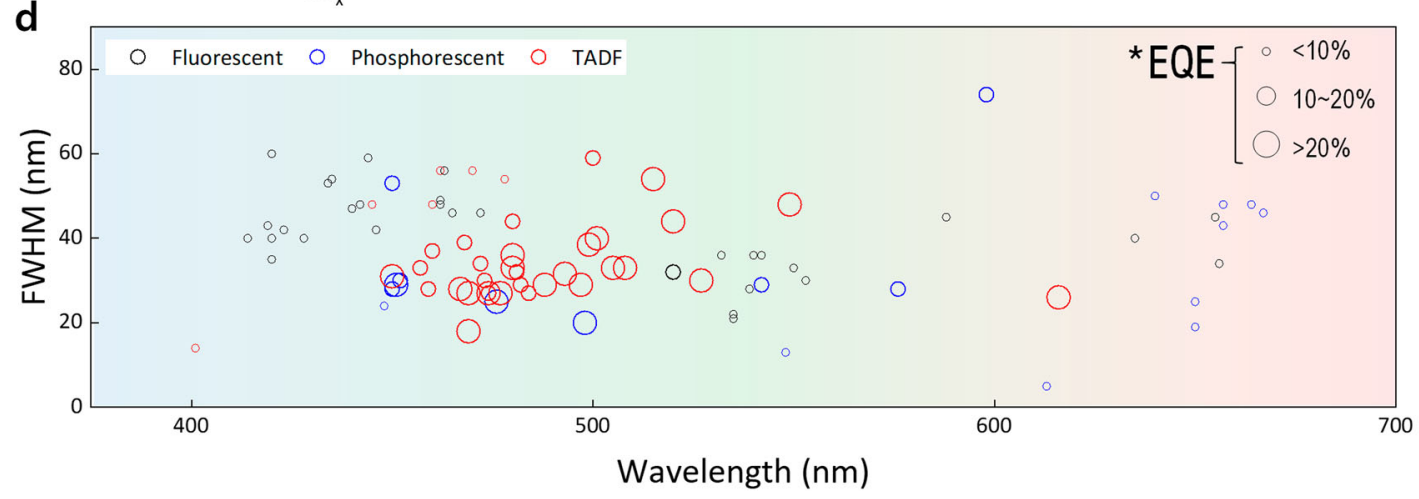

Fig. 1 Organic luminescent materials with narrowband emission. a Comparison of BT 709 and BT 2020 in CIE 1931; expanded color gamut with the vertices pushed to the parabolic edge in BT 2020. b The color gamut becomes wider with decreasing emission bandwidth. c Recent approaches in the development of organic light-emitting materials with narrowband emission based on fluorescent, phosphorescent, and TADF emitters. d Summary of FWHM and EQE values of EL spectra versus emission wavelength for fluorescent (black), phosphorescent (blue), and TADF (red) emitters.

Service Television 2020 (BT 2020) (Fig. 1) ${ }^{5}$. Compared to the previously reported BT 709, the color gamut became wider with CIE coordinates for the R, G, and B colors of (0.708, 0.292), (0.170, 0.797), and (0.131, 0.046), respectively. This change was made because of the growing demand for monochromatic R, G, and B colors to improve color purity (Fig. 1a) ${ }^{6}$.

Vibronic coupling in fluorescent and phosphorescent organic emitters between the singlet ground state $\left(\mathrm{S}_{0}\right)$ and the singlet $\left(\mathrm{S}_{1}\right)$, or triplet $\left(\mathrm{T}_{1}\right)$ excited states together with charge transfer (CT) interactions often induces broad peaks in both photoluminescence (PL) and electroluminescence (EL) spectra, showing a full width at halfmaximum (FWHM) of over $70 \mathrm{~nm}^{7-11}$. Organic emitters show significantly broader emission spectra than inorganic quantum dots (QDs) and perovskite-based emitters, limiting the potential for OLEDs with the high color purity proposed by BT $2020^{12}$. Thus, the development of OLED emitters with narrowband R, G, and B emissions with extremely small FWHM is of paramount importance. 
As seen in Fig. 1b, the color gamut becomes wider with decreasing FWHM values in the emission spectra, satisfying the color gamut standard of BT $2020^{13,14}$.

In this review, we summarize recent developments and important studies of organic light-emitting materials and devices with narrowband emission. We categorize these materials and devices based on their emission mechanism by grouping them into fluorescent, phosphorescent, and TADF emitters (Fig. 1c). Since there is currently no clear definition of "narrow emission", we mainly focused on recent reports of OLED materials with a FWHM smaller than $50 \mathrm{~nm}$ in their emission spectra (Fig. 1d). Rational molecular design strategies to achieve narrow PL and EL emission and the related electronic structure and light-emitting characteristics, including the resulting device properties, are discussed to understand the underlying mechanisms for controlling the emission bandwidth. Finally, we provide our perspective on the remaining challenges in this research area that must be overcome to develop the next generation of wide-color gamut OLED displays.

\section{Fluorescent organic light-emitting materials}

$\pi$-Conjugated organic fluorophores suffer from limited color purity due to their broad emission spectra originating from the intrinsic vibronic coupling and structural relaxation of the $S_{1}$ state (Fig. 2a). To achieve narrow EL spectra with a small FWHM, color filters and optical microcavities have been considered in the fabrication of fluorescent OLEDs ${ }^{15-17}$. However, the development of efficient organic fluorescent emitters with narrow emission bandwidths for high color purity remains an important goal. As shown in Fig. 2b, the relative intensity $\left(I_{0-1} / I_{0-0}\right)$ of the $0-0$ (between the $v=0$ vibrational levels of $\mathrm{S}_{0}$ and $\mathrm{S}_{1}$ ) and $0-1$ (from $v=0$ of $\mathrm{S}_{1}$ to $v=1$ of $\mathrm{S}_{0}$ ) vibronic transitions is determined by the Huang-Rhys factor $(S)^{18}$. When $\pi$-conjugated organic molecules have a locally excited (LE) state with a similar equilibrium geometry to the ground state (structural distortion, $\Delta Q \approx 0$ ), a large orbital overlap results in a dominant $0-0$ vibronic transition, converging the $S$ value to zero and producing a sharp single emission peak ${ }^{19}$. In contrast, an increase in $\Delta Q$ by the formation of the CT state can induce a strong contribution from 0 to $n(n=1,2,3 \ldots)$ vibronic transitions, resulting in a broader emission peak ${ }^{9,18,20}$. Thus, the design of rigid structures with a lack of CT character in the excited state is crucial to decrease the $S$ value and develop organic fluorescent materials with narrowband emission.

In this chapter, we focus on the molecular design of narrowband emitting fluorophores and structure-optical property correlations by categorizing the molecules into (i) twisted structures with bulky substituents with suppressed intermolecular aggregation, (ii) rigid/fused aromatic molecules without CT character, (iii) 5,12-dihydroquinolino[2,3-b]acridine-7,14-dione (quinacridone) and

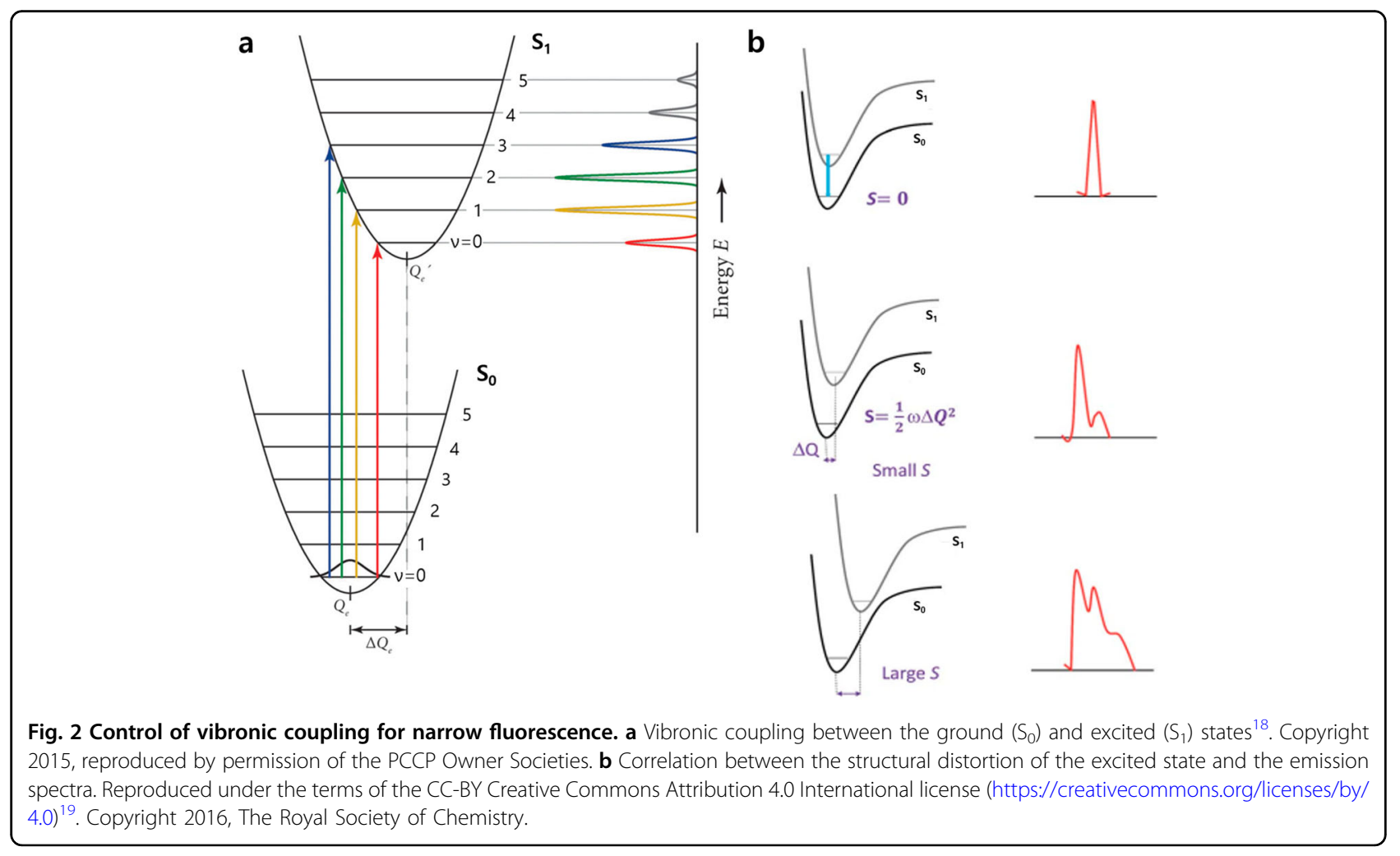


(iv) 4,4-difluoro-4-bora-3a,4a-diaza-s-indacene (BODIPY) derivatives (Fig. 3). Much of the previous work concerning fluorescent singlet emitters with narrow emission studied blue-emitting materials and their EL devices. Some quinacridone and BODIPY-based fluorophores have demonstrated green and red emission spectra with remarkably small FWHM values. The narrow emission with a predominant $0-0$ vibronic transition can be ascribed to the enhanced LE character and negligible CT interaction in the rigid and symmetrical molecular structures. The lightemitting properties of representative singlet emitters with narrowband emission and their device characteristics are summarized in Table 1.

\section{Twisted structures with bulky substituents}

Various twisted molecular structures have been designed and synthesized to suppress free rotation, vibrational splitting, and intermolecular aggregation without excimer emission in the solid state with the aim of inducing narrowband emission ${ }^{21}$. In 2001, Kwon et al. reported a twisted spirobiflourene-based anthracene derivative, 9,10bis $\left[\left(2^{\prime \prime}, 7^{\prime \prime}\right.\right.$-di-tert-butyl)-9', $9^{\prime \prime}$-spirobifluorenyl $]$ anthracene (TBSA, 1-1 $)^{22}$. TBSA showed narrow PL spectra with FWHM values of $58 \mathrm{~nm}$ at a PL maximum wavelength $\left(\lambda_{\mathrm{PL}}\right)$ of $442 \mathrm{~nm}$ in thin films. Devices with an ITO/CuPc/ $\alpha-\mathrm{NPD} / \mathrm{TBSA} / \mathrm{Alq}_{3} / \mathrm{LiF} / \mathrm{Al}$ configuration (full names of the abbreviations in the device architecture can be found in each table) showed a peak in the EL spectrum at $442 \mathrm{~nm}$ with an FWHM of $48 \mathrm{~nm}$. By combining a rigid anthracene core with twisted xylene substituents, Kim et al. reported 9,10-bis(2,5-dimethyl-4-(naphthalen-2-yl)phenyl) anthracene (BDNA, 1-2) and 9,10-bis(2,5-dimethylbiphenyl-4-yl)anthracene (BDPA, 1-3 ${ }^{23}$. Because of the twisted conformation of the xylene substituents relative to the anthracene core, vibronic coupling and self-quenching effects are suppressed, and the highest occupied molecular orbital (HOMO) and lowest unoccupied molecular orbital (LUMO) are localized mainly at the core. The PL spectra of BDNA and BDPA have small FWHM values of 19 and $18 \mathrm{~nm}$ at $\lambda_{\mathrm{PL}}=431$ and $426 \mathrm{~nm}$ in the thin film, respectively. Similarly, Anthony et al. synthesized an ethynylated tetracene derivative, 5,12-dimethoxy-6,11-bis (5-triisopropylsilylthienylethynyl)tetracene (1-4), which showed narrow PL emission with a small Stokes shift (20 $\mathrm{nm})$ due to its rigid molecular structure ${ }^{24}$. The ITO/TPD/ 1-4/LiF/Al EL device displayed a sharp emission at 656 $\mathrm{nm}$ with an FWHM of $34 \mathrm{~nm}$ and did not exhibit substantial aggregate formation.

To prevent intermolecular packing, Park et al. designed a series of blue-emissive indenopyrazine (IPY) derivatives with terphenyl side groups, 6,6,12,12-tetraethyl-2,8-bis$\left[1,1^{\prime} ; 3^{\prime}, 1^{\prime \prime}\right]$-terphenyl-4'-yl-6,12-dihydro-diindeno[1,2b;1', $2^{\prime}$-e]pyrazine ( $m$-TP-EPY, 1-5), 6,6,12,12-tetraethyl1,7-bis-[1,1';3', $\left.1^{\prime \prime}\right]$ terphenyl-4'-yl-6,12-dihydro-diindeno [1,2-b;1', $2^{\prime}$-e]pyrazine (o-TP-EPY, 1-6), and 6,6,12,12tetraethyl-3,9-bis-[1,1'; $\left.3^{\prime}, 1^{\prime \prime}\right]$ terphenyl-4'-yl-6,12-dihydrodiindeno $\left[1,2-\mathrm{b} ; 1^{\prime}, 2^{\prime}\right.$-e $]$ pyrazine ( $p$-TP-EPY, 1-7 $)^{25-27}$. The twisted molecular structure restricts the $\pi$-conjugation between the IPY core and terminal terphenyl groups, resulting in narrow blue PL spectra with small FWHM values between 40 and $50 \mathrm{~nm}$ in both chloroform and thin films. Based on a new phenanthro[9,10-d]imidazole (PI) building block, $\mathrm{Ma}$ and $\mathrm{Lu}$ et al. synthesized blue-emissive 1,2-diphenyl-phenanthro[9,10-d]imidazole (PPI, 1-8) ${ }^{28}$. In tetrahydrofuran (THF), a narrow PL band at $\lambda_{\mathrm{PL}}=369$ $\mathrm{nm}$ was measured with an FWHM of $34 \mathrm{~nm}$ and PL quantum yield (PLQY) of 70\%. Subsequently, Lu further developed three PPI derivatives, (2-(4-(9H-carbazol-9-yl) phenyl)-1-phenyl-1H-phenanthro[9,10-d]imidazole) (M1, 1-9), 1-phenyl-2-(4-(3-(1-phenyl- $1 H$-phenanthro[9,10-d] imidazol-2-yl)-9H-carbazol-9-yl)phenyl)- $1 H$-phenanthro [9,10-d]imidazole (M2, 1-10), and 1-phenyl-2-(4-(triphenylsilyl)phenyl)-3a,11b-dihydro- $1 H$-phenanthro[9,10d]imidazole (SiPIM, 1-11) ${ }^{29,30}$. Similar PL spectra with a small FWHM of approximately $40 \mathrm{~nm}$ were measured in various solvents with different polarities, indicating the absence of intramolecular charge transfer (ICT) characteristics in these compounds. In particular, M1- and M2-based OLEDs with a device architecture of ITO/NPB/ $\mathrm{M} 1$ or $\mathrm{M} 2 / \mathrm{TPBI} / \mathrm{LiF} / \mathrm{Al}$ exhibited narrow EL spectra (FWHM $\sim 40 \mathrm{~nm}$ ) with CIE coordinates of $(0.165,0.050)$ and $(0.166,0.056)$, respectively.

The light-emitting properties of a new donor-acceptor type triphenyl-substituted imidazole moiety, $\mathrm{N}, \mathrm{N}$-diphenyl-4'-(1,4,5-triphenyl-1 $H$-imidazol-2-yl)-[1,1'-biphenyl]4-amine (TPA-PIM, 1-12), were studied by Yang et al. ${ }^{31}$. A fully twisted TPA-PIM showed highly suppressed vibration splitting and narrow emission spectra in the solid state. TPA-PIM devices with a configuration of ITO/ PEDOT:PSS/NPB/TCTA/TPA-PIM/TPBi/LiF/Al showed an extraordinarily narrow EL emission at $420 \mathrm{~nm}$ with an FWHM of $35 \mathrm{~nm}$ and an external quantum efficiency (EQE) of $3.28 \%$ with CIE coordinates of $(0.161,0.046)$ (Fig. 4a). Gong et al. also reported a series of deep-blueemissive materials based on a spiro[fluorene-7,9'-benzofluorene] core, including $N, N, N, N^{\prime}$-tetraphenylspiro [fluorene-7,9'-benzofluorene] (SFBF)-5,9-diamine (BD6DPA, 1-13), $N, N^{\prime}$-di-(2-naphthyl)- $N, N^{\prime}$-diphenyl-SFBF5,9-diamine (BD-6NPA, 1-14), $N, N^{\prime}$-diphenyl- $N, N^{\prime}$-di- $m$ tolyl-SFBF-5,9-diamine (BD-6MDPA, 1-15), and $N, N$ '-diphenyl- $N, N$ '-bis(4-(trimethylsilyl)phenyl)-SFBF-5,9-

diamine (BD-6TMSA, 1-16) ${ }^{32}$. Spiro compounds with specific steric configurations can prevent intermolecular interactions with amorphous morphology and high PLQY, even in films. The corresponding EL devices showed narrow EL bands at $460 \mathrm{~nm}(\mathrm{FWHM}=46-56 \mathrm{~nm})$ with EQEs of $6.54-8.16 \%$. In addition, a blue-emissive indenofluorenyl core with two spiro-linked xanthenyl units, 


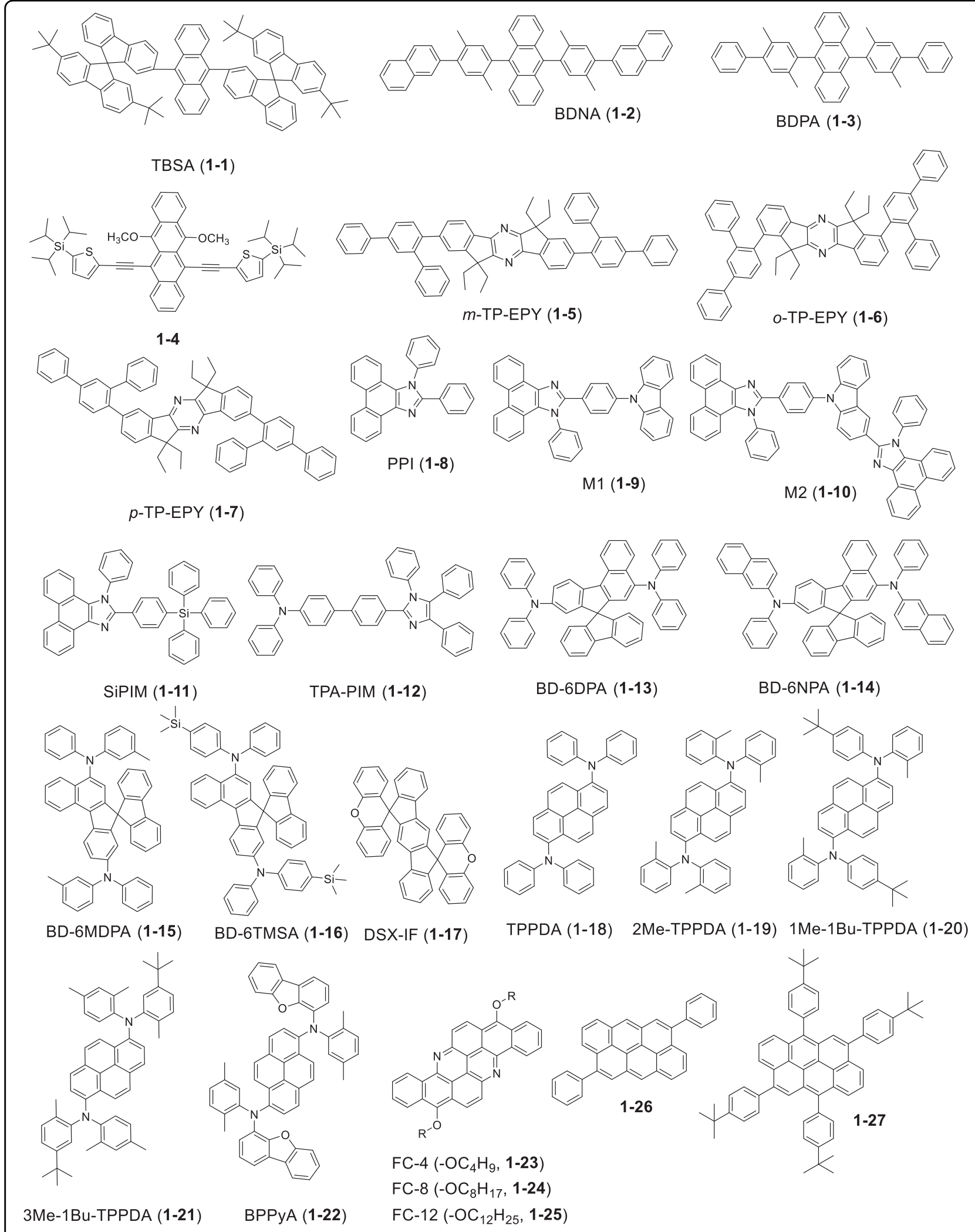

Fig. 3 (See legend on next page.) 


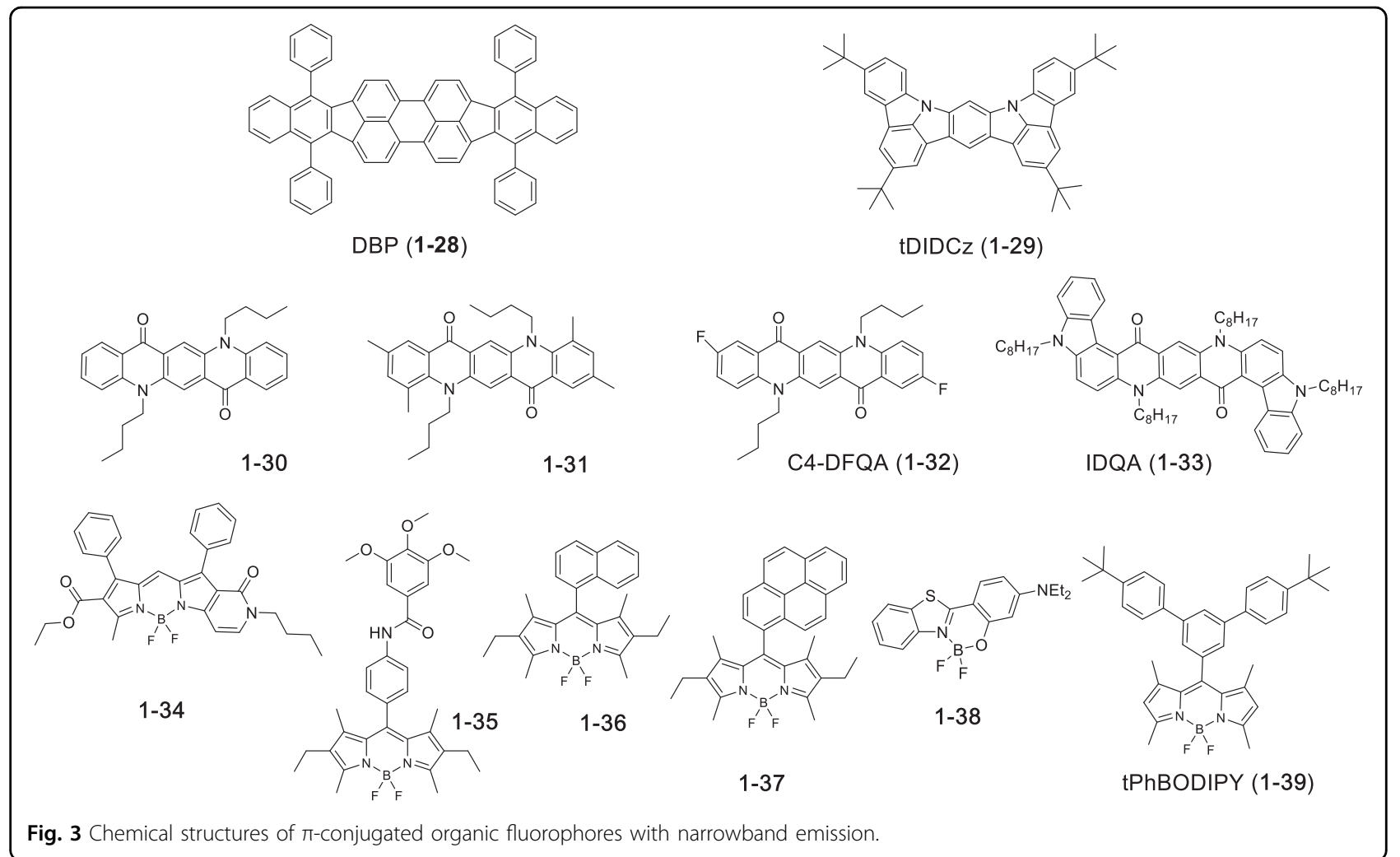

dispiro[xanthene-9,6-indeno[1,2-b]fluorene-12,9'-xanthene] (DSX-IF, 1-17), was reported by Rault-Berthelot et al. ${ }^{33}$. Owing to the highly rigid spiro structures, DSX-IF suppressed excimer formation in the solid state with improved solubility and narrow PL (in cyclohexane) and EL spectra with FWHM values of $\sim 30$ and $40 \mathrm{~nm}$, respectively.

In 2018, Park et al. designed four different kinds of narrow blue-emitting fluorophores based on a pyrene moiety by varying diphenylamine (DPA) side groups ${ }^{34}$. To hinder intermolecular packing, they introduced different kinds of alkyl side chains onto the DPA moiety and synthesized $N, N$, $N^{\prime}, N^{\prime}$-tetraphenyl-pyrene-1,6-diamine (TPPDA, 1-18), $N, N$, $N^{\prime}, N^{\prime}$-tetra-o-tolylpyrene-1,6-diamine (2Me-TPPDA, 1-19), $N, N^{\prime}$-bis(4-(tert-butyl)phenyl)- $N, N^{\prime}$-di-o-tolylpyrene-1,6-diamine (1Me-1Bu-TPPDA, 1-20), and $N, N^{\prime}$-bis(5-(tert-butyl)2-methylphenyl)- $N, N^{\prime}$-bis(2,4-dimethylphenyl)-pyrene-1,6diamine (3Me-1Bu-TPPDA, 1-21). In toluene, the PL maxima (FWHM) of TPPDA, 2Me-TPPDA, 1Me-1BuTPPDA, and 3Me-1Bu-TPPDA were 458 (38), 452 (39), 462 (39), and $461 \mathrm{~nm}(41 \mathrm{~nm})$, respectively. A higher PLQY was measured for 1Me-1Bu-TPPDA (89\%) and 3Me-1BuTPPDA (91\%) than for TPPDA (87\%) and 2Me-TPPDA (79\%) because bulky tert-butyl substituents can prevent intermolecular aggregation. All EL devices with a configuration of ITO/2-TNATA/NPB/ $\alpha, \beta-A D N: 4 \%$ dopant $/ \mathrm{Alq}_{3} /$ $\mathrm{LiF} / \mathrm{Al}$ showed a narrow emission with FWHM $=\sim 40 \mathrm{~nm}$.
In particular, 3Me-1Bu-TPPDA showed the highest EQE of $9.25 \%$ with a small y value for the CIE coordinate $(0.133$, $0.145)$. Kwon et al. also synthesized a pyrene-based blue fluorophore, $N, N^{\prime}$-bis-dibenzofuran-4-yl- $N, N^{\prime}$-bis-(2,5-dimethyl-phenyl)-pyrene-1,6-diamine (BPPyA, 1-22), and reported a TADF host-sensitization system using the BPPyA emitter and 9,9-dimethyl-2,7-di(10H-spiro[acridine-9,9'fluoren]-10-yl)-9H-thioxanthene-10,10-dioxide (SPACDMT) as a TADF sensitizer, showing $\lambda_{\mathrm{EL}}=455 \mathrm{~nm}$ with a FWHM of $43 \mathrm{~nm}$ and an EQE of $7 \%{ }^{35}$.

\section{Fused aromatic compounds with structural symmetry}

Symmetrically structured fused aromatic compounds without ICT character have demonstrated narrow emission with similar molecular geometry in the $S_{0}$ and $\mathrm{S}_{1}$ states. For example, flavanthrone is a well-known anthraquinone-type fluorophore with a rigid planar backbone, and several flavanthrone derivatives have been investigated in OLEDs where a dominant LE emission was observed with a high oscillator strength. Based on the flavanthrone core, Monkman et al. synthesized a series of 8,16-dialkoxybenzo[h]-benz[5,6] acridino[2,1,9,8-klmna] acridines (FCs) with various alkoxy side chains $\left(-\mathrm{OC}_{4} \mathrm{H}_{9}(\mathrm{FC}-4,1-23),-\mathrm{OC}_{6} \mathrm{H}_{13}\right.$ (FC-6), $-\mathrm{OC}_{8} \mathrm{H}_{17}$ (FC-8, 1-24), $-\mathrm{OC}_{10} \mathrm{H}_{21}$ (FC-10), and $\left.-\mathrm{OC}_{12} \mathrm{H}_{25}(\mathrm{FC}-12, \mathbf{1 - 2 5})\right)^{36}$. All FC derivatives showed similar optical properties ( $\lambda_{\mathrm{PL}}$ of $521 \mathrm{~nm}$ in chloroform) 
Table 1 Summary of optical and device properties of fluorescent emitters.

\begin{tabular}{|c|c|c|c|c|c|c|c|c|c|}
\hline No. & Emitter & $\lambda_{\mathrm{PL}}(\mathrm{nm})$ & FWHM $_{\mathrm{PL}}(\mathrm{nm})$ & PLQY (\%) & $\lambda_{\mathrm{EL}}(\mathrm{nm})$ & FWHM $_{\mathrm{EL}}(\mathrm{nm})$ & EQE (\%) & $\mathrm{CIE}_{x, y}$ & Refs. \\
\hline \multicolumn{10}{|c|}{ Twisted structures with bulky substituents } \\
\hline $1-1$ & TBSA $^{\mathrm{a}}$ & $\begin{array}{l}443 \text { (in CF) } \\
442 \text { (in film) }\end{array}$ & $\begin{array}{l}63^{*} \text { (in CF) } \\
58^{*} \text { (in film) }\end{array}$ & NA & 442 & 48 & NA & $(0.14,0.08)$ & 22 \\
\hline $1-2$ & $B D N A^{b}$ & $\begin{array}{l}408 \text { (in CF) } \\
431 \text { (in film) }\end{array}$ & $\begin{array}{l}42^{*} \text { (in CF) } \\
19^{*} \text { (in film) }\end{array}$ & NA & 435 & 54 & 3.94 & $(0.157,0.058)$ & 23 \\
\hline $1-3$ & $\mathrm{BDPA}^{\mathrm{b}}$ & $\begin{array}{l}411 \text { (in CF) } \\
426 \text { (in film) }\end{array}$ & $\begin{array}{l}40^{*} \text { (in CF) } \\
18^{*} \text { (in film) }\end{array}$ & NA & 434 & 53 & 3.23 & $(0.156,0.046)$ & \\
\hline $1-4$ & $8^{c}$ & 637 (in Hex) & $53^{*}$ (in Hex) & 36 (in Hex) & 656 & $34^{*}$ & NA & NA & 24 \\
\hline $1-5$ & $m$-TP-EPY & $\begin{array}{l}437 \text { (in CF) } \\
443 \text { (in film) }\end{array}$ & $\begin{array}{l}45^{*} \text { (in CF) } \\
54 \text { (in film) }\end{array}$ & 73 (in CF) & 440 & 47 & 4.61 & $(0.157,0.079)$ & $25-27$ \\
\hline $1-6$ & o-TP-EPY & $\begin{array}{l}411 \text { (in CF) } \\
418 \text { (in film) }\end{array}$ & $\begin{array}{l}42^{*} \text { (in CF) } \\
43 \text { (in film) }\end{array}$ & 42 (in CF) & 419 & 43 & 1.27 & $(0.175,0.068)$ & 25 \\
\hline $1-7$ & $p$-TP-EPY & $\begin{array}{l}415 \text { (in CF) } \\
426 \text { (in film) }\end{array}$ & $\begin{array}{l}42^{*} \text { (in CF) } \\
43 \text { (in film) }\end{array}$ & 61 (in CF) & 423 & 42 & 1.88 & $(0.173,0.063)$ & \\
\hline $1-8$ & $\mathrm{PPI}^{\mathrm{e}}$ & $\begin{array}{l}369 \text { (in THF) } \\
395 \text { (in film) }\end{array}$ & $\begin{array}{l}34^{*} \text { (in THF) } \\
50^{*} \text { (in film) }\end{array}$ & $\begin{array}{l}70 \text { (in THF) } \\
40 \text { (in film) }\end{array}$ & 451 & NA & 0.7 & $(0.16,0.17)$ & 28 \\
\hline $1-9$ & $M 1^{f}$ & $\begin{array}{l}381 \text { (in THF) } \\
414 \text { (in film) }\end{array}$ & $\begin{array}{l}40 \text { (in THF) } \\
61^{*} \text { (in film) }\end{array}$ & 65 (in film) & 420 & 40 & 1.94 & $(0.165,0.050)$ & 29 \\
\hline $1-10$ & $M 2^{f}$ & $\begin{array}{l}407 \text { (in THF) } \\
407 \text { (in film) }\end{array}$ & $\begin{array}{l}40 \text { (in THF) } \\
55^{*} \text { (in film) }\end{array}$ & 59 (in film) & 428 & 40 & 3.02 & $(0.166,0.056)$ & \\
\hline $1-11$ & SiPIM $^{9}$ & $\begin{array}{l}372 \text { (in THF) } \\
427 \text { (in film) }\end{array}$ & $\begin{array}{l}44 \text { (in THF) } \\
63^{*} \text { (in film) }\end{array}$ & 81 (in film) & 420 & 60 & 6.29 & $(0.163,0.040)$ & 30 \\
\hline $1-12$ & TPA-PIM ${ }^{h}$ & 424 (in THF) & $56^{*}$ (in THF) & $\begin{array}{l}75 \text { (in CF) } \\
60 \text { (in film) }\end{array}$ & 420 & 35 & 3.28 & $(0.161,0.046)$ & 31 \\
\hline $1-13$ & BD-6DPA ${ }^{i}$ & $\begin{array}{l}478 \text { (in solution) } \\
484 \text { (in film) }\end{array}$ & NA & 74.7 (in solution) & 463 & 56 & 6.54 & $(0.135,0.175)$ & 32 \\
\hline $1-14$ & BD-6NPA & $\begin{array}{l}464 \text { (in solution) } \\
491 \text { (in film) }\end{array}$ & NA & 65.6 (in solution) & 465 & 46 & 7.32 & $(0.134,0.168)$ & \\
\hline $1-15$ & BD-6MDPA' & $\begin{array}{l}468 \text { (in solution) } \\
487 \text { (in film) }\end{array}$ & NA & 73.6 (in solution) & 462 & 48 & 8.16 & $(0.135,0.156)$ & \\
\hline $1-16$ & BD-6TMSA & $\begin{array}{l}459 \text { (in solution) } \\
484 \text { (in film) }\end{array}$ & NA & 67.2 (in solution) & 462 & 49 & 7.45 & $(0.135,0.151)$ & \\
\hline $1-17$ & DSX-IF' & $\begin{array}{l}347 \text { (in Cyhex) } \\
373^{*} \text { (in film) }\end{array}$ & $\begin{array}{l}30^{*} \text { (in Cyhex) } \\
60^{*} \text { (in film) }\end{array}$ & 63 (in Cyhex) & 414 & $40^{*}$ & NA & $(0.19,0.08)$ & 33 \\
\hline $1-18$ & TPPDA $^{k}$ & $\begin{array}{l}458 \text { (in toluene) } \\
534 \text { (in PMMA film) }\end{array}$ & $\begin{array}{l}38 \text { (in toluene) } \\
84 \text { (in PMMA film) }\end{array}$ & $\begin{array}{l}87 \text { (in } \\
\text { PMMA film) }\end{array}$ & 462 & 40 & 8.34 & $(0.137,0.142)$ & 34 \\
\hline $1-19$ & 2Me-TPPDA ${ }^{k}$ & $\begin{array}{l}452 \text { (in toluene) } \\
526 \text { (in PMMA film) }\end{array}$ & $\begin{array}{l}39 \text { (in toluene) } \\
116 \text { (in PMMA film) }\end{array}$ & $\begin{array}{l}79 \text { (in } \\
\text { PMMA film) }\end{array}$ & 457 & 40 & 7.57 & $(0.138,0.121)$ & \\
\hline $1-20$ & $\begin{array}{l}\text { 1Me-1Bu- } \\
\text { TPPDA }^{\mathrm{k}}\end{array}$ & $\begin{array}{l}462 \text { (in toluene) } \\
468 \text { (in PMMA film) }\end{array}$ & $\begin{array}{l}39 \text { (in toluene) } \\
54 \text { (in PMMA film) }\end{array}$ & $\begin{array}{l}89 \text { (in } \\
\text { PMMA film) }\end{array}$ & 466 & 39 & 8.47 & $(0.128,0.178)$ & \\
\hline $1-21$ & $\begin{array}{l}\text { 3Me-1Bu- } \\
\text { TPPDA }^{\mathrm{k}}\end{array}$ & $\begin{array}{l}461 \text { (in toluene) } \\
465 \text { (in PMMA film) }\end{array}$ & $\begin{array}{l}41 \text { (in toluene) } \\
45 \text { (in PMMA film) }\end{array}$ & $\begin{array}{l}91 \text { (in } \\
\text { PMMA film) }\end{array}$ & 463 & 37 & 9.25 & $(0.133,0.145)$ & \\
\hline $1-22$ & BPPyA' $^{\prime}$ & 458 (in toluene) & $38^{*}$ (in toluene) & 98.5 (in toluene) & $455^{*}$ & $43^{*}$ & 7.0 & $(0.14,0.11)$ & 35 \\
\hline \multicolumn{10}{|c|}{ Fused aromatic compounds with structural symmetry } \\
\hline $1-23$ & $\mathrm{FC}-4^{\mathrm{m}}$ & NA & NA & NA & $535^{*}$ & $21^{*}$ & 0.9 & NA & 36 \\
\hline $1-24$ & $F C-8^{m}$ & $520^{*}$ (in CF) & $55^{*}$ (in CF) & 80 (in CF) & $535^{*}$ & $22^{*}$ & 0.9 & NA & \\
\hline $1-25$ & $\mathrm{FC}-12^{\mathrm{m}}$ & NA & NA & NA & $535^{*}$ & $22^{*}$ & NA & NA & \\
\hline $1-26$ & $2^{n}$ & $\begin{array}{l}442 \text { (in DCM) } \\
439 \text { (in PMMA film) }\end{array}$ & $\begin{array}{l}14^{*} \text { (in DCM, and } \\
\text { PMMA film) }\end{array}$ & $\begin{array}{l}33 \text { (in DCM) } \\
49 \text { (in } \\
\text { PMMA film) }\end{array}$ & 446 & $42^{*}$ & NA & $(0.15,0.14)$ & 37 \\
\hline $1-27$ & $5^{n}$ & $\begin{array}{l}467 \text { (in DCM) } \\
468 \text { (in PMMA film) }\end{array}$ & NA & $\begin{array}{l}27 \text { (in DCM) } \\
47 \text { (in } \\
\text { PMMA film) }\end{array}$ & 472 & $46^{*}$ & NA & $(0.13,0.25)$ & \\
\hline $1-28$ & $\mathrm{DBP}^{\circ}$ & $\begin{array}{l}603^{*} \text { (in DCM) } \\
596 \text { (in Ben) }\end{array}$ & $\begin{array}{l}63^{*} \text { (in DCM) } \\
22^{*} \text { (in Ben) }\end{array}$ & $\begin{array}{l}60 \text { (in DCM) } \\
85 \text { (in Ben) }\end{array}$ & 610 & $25^{*}$ & 8.0 & $(0.61,0.38)$ & 38,39 \\
\hline $1-29$ & $\mathrm{tDIDCz} z^{p}$ & 393 (in THF) & 20 (in THF) & 60 (in PS film) & 401 & 14 & 2.75 & $(0.16,0.02)$ & 40 \\
\hline \multicolumn{10}{|c|}{ Quinacridone derivatives } \\
\hline $1-30$ & $1^{9}$ & $\begin{array}{l}538 \text { (in CF) } \\
542 \text { (in Alq } \text { film) }\end{array}$ & $\begin{array}{l}24^{*} \text { (in CF) } \\
28^{*}\left(\text { in } \text { Alq }_{3} \text { film }\right)\end{array}$ & 91 (in CF) & $539^{*}$ & $28^{*}$ & NA & $(0.32,0.65)$ & 41 \\
\hline $1-31$ & $2^{a}$ & $\begin{array}{l}532^{*} \text { (in CF) } \\
536 \text { (in Alq } \text { film) }^{2}\end{array}$ & $\begin{array}{l}26^{*} \text { (in CF) } \\
34^{*} \text { (in Alq } \text { film }_{3}\end{array}$ & 90 (in CF) & $532^{*}$ & $36^{*}$ & NA & $(0.34,0.63)$ & \\
\hline $1-32$ & $\mathrm{C}_{4}-\mathrm{DFQA}{ }^{r}$ & 535 (in THF) & $21^{*}$ (in THF) & 97 (in THF) & $553^{*}$ & $30^{*}$ & NA & $(0.42,0.56)$ & 42 \\
\hline
\end{tabular}


Table 1 continued

\begin{tabular}{|c|c|c|c|c|c|c|c|c|c|}
\hline No. & Emitter & $\lambda_{\mathrm{PL}}(\mathrm{nm})$ & $\mathrm{FWHM}_{\mathrm{PL}}(\mathrm{nm})$ & PLQY (\%) & $\lambda_{\mathrm{EL}}(\mathrm{nm})$ & $\mathrm{FWHM}_{\mathrm{EL}}(\mathrm{nm})$ & EQE (\%) & $\mathrm{ClE}_{x, y}$ & Refs. \\
\hline $1-33$ & $\mathrm{IDQA}^{\mathrm{s}}$ & 568 (in Tol) & $25^{*}$ (in Tol) & 91 (in Tol) & 588 & $45^{*}$ & NA & NA & 43 \\
\hline \multicolumn{10}{|c|}{ BODIPY derivatives } \\
\hline $1-34$ & $2^{t}$ & $\begin{array}{l}618 \text { (in DCM) } \\
655^{*} \text { (in film) }\end{array}$ & $\begin{array}{l}48^{*} \text { (in DCM) } \\
113^{*} \text { (in film) }\end{array}$ & $\begin{array}{l}86 \text { (in DCM) } \\
7 \text { (in film) }\end{array}$ & 655 & 45 & 2.0 & $(0.55,0.28)$ & 44,45 \\
\hline $1-35$ & 3 & $\begin{array}{l}540 \text { (in DCM) } \\
545,633 \text { (in film) }\end{array}$ & $31^{*}$ (in DCM) & 65 (in DCM) & $\begin{array}{l}550^{\mathrm{u}} \\
635^{\mathrm{v}}\end{array}$ & $\begin{array}{l}33^{*, \mathrm{u}} \\
40^{*, v}\end{array}$ & NA & NA & 46 \\
\hline $1-36$ & $2^{w}$ & $\begin{array}{l}544 \text { (in DCM and } \\
\text { PMMA film) }\end{array}$ & $27^{*}$ (in DCM) & 99 (in DCM) & 542 & $36^{*}$ & NA & NA & 47 \\
\hline $1-37$ & $4^{w}$ & $\begin{array}{l}543 \text { (in DCM and } \\
\text { PMMA film) }\end{array}$ & $26^{*}$ (in DCM) & 99 (in DCM) & 540 & $36^{*}$ & NA & NA & \\
\hline $1-38$ & $3 b^{x}$ & $\begin{array}{l}434 \text { (in DCM) } \\
463 \text { (in PMMA film) }\end{array}$ & $\begin{array}{l}36^{*} \text { (in DCM) } \\
38 \text { (in PMMA film) }\end{array}$ & $\begin{array}{l}96 \text { (in DCM) } \\
61 \text { (in } \\
\text { PMMA film) }\end{array}$ & $444^{*}$ & $59^{*}$ & NA & $(0.15,0.11)$ & 48 \\
\hline $1-39$ & tPhBODIPYy & 518 (in Tol) & 28 (in Tol) & 98 (in Tol) & 520 & 32 & 19 & $(0.26,0.67)$ & 49 \\
\hline
\end{tabular}

CF chloroform, Hex hexane, Cyhex cyclohexane, DCM dichloromethane, PMMA poly(methyl methacrylate), Tol toluene, Ben benzene, ITO indium tin oxide, CuPC copper phthalocyanine, $a-N P D=N P B \quad N, N^{\prime}$-diphyenyl- $N, N^{\prime}$-bis(1-naphthyl)-1, $1^{\prime}$-biphenyl-4, $4^{\prime \prime}$-diamine, Alq ${ }_{3}$ tris(8-hydroxyquinoline) aluminum, 2-TNATA 4,4',4"-tris( $N$-(2naphthyl)- $N$-phenyl-amino)-triphenylamine, TCTA tris(4-carbazoyl-9-ylphenyl)amine, TPBi 2,2',2"'-Benzene-1,3,5-triyltris(1-phenyl-1 H-benzimidazole), TAPC 1,1-bis[(di-4tolyl-amino)phenyl]cyclohexane, TMPyPB 1,3,5-tri( $m$-pyridin-3-ylphenyl)benzene, PEDOT:PSS poly(3,4-ethylene dioxythiophene):poly(styrene sulfonate), $a, \beta-A N D$ 9(naphthalen-1-yl)-10-(naphthalen-2-yl)anthracene, HATCN 1,4,5,8,9,12-hexaazatriphenylene-hexacarbonitrile, $D N T P D N, N^{\prime}$-diphenyl- $N, N^{\prime}$-bis-[4-(phenyl-m-tolyl-amino)phenyl]-biphenyl-4,4'-diamine, DCDPA 3,5-di(9H-carbazol-9-yl)- $\mathrm{N}, \mathrm{N}$-diphenylaniline, DBFPO 2,8-bis(diphenylphosphine oxide) dibenzofuran, SPAC-DMT 9,9-dimethyl2,7-di(10H-spiro[acridine-9,9'-fluoren]-10-yl)-9H-thioxanthene-10,10-dioxide, TSPO1 diphenyl[4-(triphenylsilyl)phenyl]phosphine oxide, MADN 2-methyl-9,10-di(2naphthyl) anthracene, PVK poly(9-vinylcarbazole), CBP 4,4'-bis( $N$-carbazolyl)-1,1'-biphenyl, Bphen 4,7-diphenyl-1,10-phenanthroline, TBADN 2-tert-butyl-9,10-di (naphth-2-yl)anthracene, TPD $N, N^{\prime}$-bis(3-methylphenyl)- $N, N^{\prime}$-diphenylbenzidine, $B C P$ 2,9-dimethyl-4,7-diphenyl1,1'-phenanthroline, $P F I$ tetrafluoroethylene-perfluoro3,6-dioxa-4-methyl-7-octenesulphonic acid, TFB poly[2,7-(9,9-di-n-octylfluorene)-alt-(1,4-phenylene-[(4-sec-butylphenyl)amino]-1,4-phenylene)], $m C P$ 1,3-bis( $N$-carbazolyl)benzene, DCzDCN 3',5'-di(9H-carbazol-9-yl)-[1,1'-biphenyl]-3,5-dicarbonitrile, 4CzIPN 2,4,5,6-tetra(9H-carbazol-9-yl)isophthalonitrile, DPPyA 9,10-bis(6-phenylpyridin-3-yl)anthracene, $P C z A c$ 9,9-dimethyl-10-(9-phenyl-9H-carbazol-3-yl)-9,10-dihydroacridine.

*Estimated values based on the graph (not provided by author).

${ }^{a}$ ITO/CuPc/a-NPD/emitter/Alq $3 /$ LiF/Al.

bITO/NPB/TCTA/emitter/Bphen/LiF/Al.

CITO/TPD/emitter/LiF/Al.

${ }_{\text {ITO/2-TNATA/NPB/TCTA/emitter/Alq }} /$ LiF/AI.

e ITO/NPB/emitter/TPBi/Alq $/$ /LiF/Al.

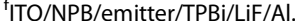

9ITO/ $\mathrm{MoO}_{3} / \mathrm{TAPC} /$ emitter (vacuum-deposition)/TmPyPb/LiF/Al.

hITO/PEDOT:PSS/NPB/TCTA/emitter/TPBi/LiF/Al.

ITO/DNTPD/NPB/MADN:5\% emitter/Alq ${ }_{3} / \mathrm{Li} / \mathrm{Al}$.

j।TO/PEDOT:PSS/NPB/emitter/Ca.

kITO/2-TNATA/NPB/a, $\beta$-ADN:4\% emitter/Alq3/LiF/Al.

ITO/HATCN/TAPC/DCDPA/DBFPO:SPAC-DMT:0.7\% emitter/TPBi/LiF/Al.

mITO/PEDOT:PSS/PVK/CBP:1 wt\% emitter/TPBi/LiF/Al.

${ }^{n}$ ITO/NPB/TBADN:1.1\% emitter/Alq ${ }_{3} / \mathrm{MgAg}$.

ITO/PEDOT:PSS/CBP:15 wt\% DC-TC:CBP:2 wt\% emitter/TmPyPB/LiF/AI.

pITO/NPB/TCTA/PCzAc/mCP/mCP:TSPO1 (1:1):1 wt\% emitter/TSPO1/TPBi/LiF/Al.

qITO/CuPc/NPB/Alq3:0.5\% emitter/LiF/Al.

'ITO/NPB/Alq 3 :0.5\% emitter/Alq $3 /$ LiF/AI.

${ }^{5} \mathrm{TTO} / \mathrm{NPB} / \mathrm{Alq}_{3}: 1 \mathrm{wt} \%$ emitter/Alq 3 /LiF/Al.

tITO/Cul/CBP:20\% emitter/Bphen/Ca/Al.

uITO/a-NPD/Alq $3: 7$ mol\% emitter/BCP/Al.

vITO/a-NPD/Alq ${ }_{3}: 19 \mathrm{~mol} \%$ emitter/BCP/AI.

wITO/PVK:5 wt\% emitter/BPhen/Alq $\mathrm{A}_{3} / \mathrm{Yb}$.

xITO/PEDOT:PSS:PFI/TFB/CBP:3 wt\% emitter/TPBi/LiF/AI

y ITO/TAPC/TCTA/mCP/DCzDCN:30 wt\% 4CzIPN:1 wt\% emitter/DCzDCN/DPPyA:Liq./LiF/AI.

because of the identical core structure. In particular, a Stokes shift of only $9 \mathrm{~nm}$ was observed for $\mathrm{FC}-8$ in chloroform, suggesting a negligible geometrical distortion between the ground and excited states. When FC4, FC-8, and FC-12 OLEDs were fabricated with a configuration of ITO/PEDOT:PSS/PVK/CBP:1 wt\% FC4,8 , or $12 / \mathrm{TPBi} / \mathrm{LiF} / \mathrm{Al}$, remarkably narrow EL spectra were measured with an EL maximum wavelength $\left(\lambda_{\mathrm{EL}}\right)$ of $535 \mathrm{~nm}$ and an extremely small FWHM (21-22 nm) (Fig. 4b).

A series of rigid anthanthrene derivatives were synthesized by Neckers et al. with various phenyl substituents, including 4,10-diphenylanthanthrene (1-26) and p-tertbutylphenyl-4,6,10,12-tetraphenylanthanthrene $(\mathbf{1}-\mathbf{2 7})^{37}$. Compounds 1-26 showed a remarkably small FWHM of $14 \mathrm{~nm}$ with a $\lambda_{\mathrm{PL}}$ of $442 \mathrm{~nm}$ and a PLQY of $33 \%$ in dichloromethane (DCM). Because anthanthrene in DCM had almost isoenergetic $S_{1}$ and $T_{2}$ states, nonradiative relaxation pathways via intersystem crossing (ISC) decreased the PL efficiency. However, in the solid state, the $T_{2}$ state had a slightly higher energy than the $S_{1}$ state, making the nonradiative ISC process far less likely to occur and yielding blueshifted emission with a higher PLQY compared to that in solution. OLED devices using 


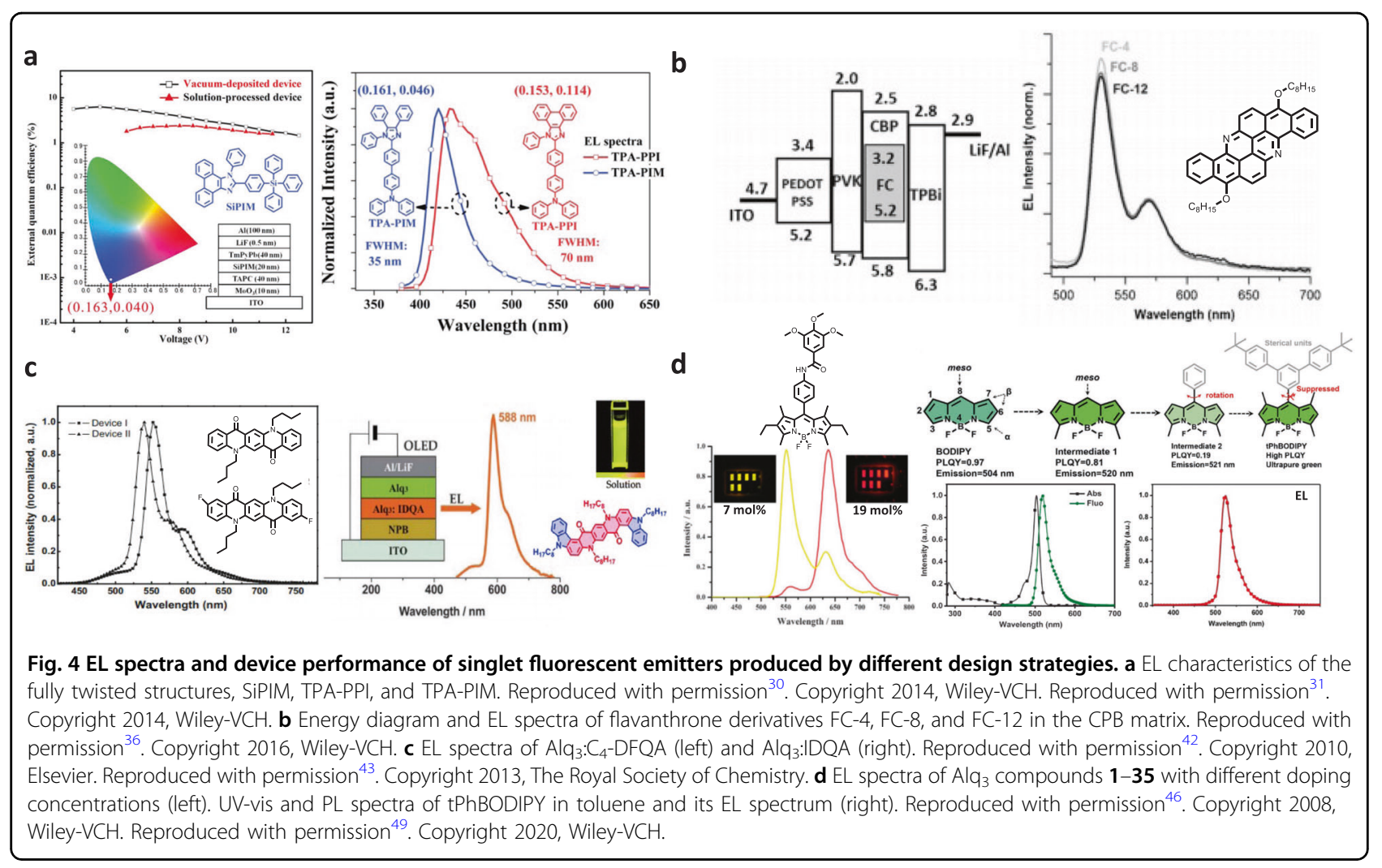

compounds 1-26 and 1-27 with a structure of ITO/NPB/ TBADN:1.1\% 1-26 or 1-27/Alq $3 / \mathrm{MgAg}$ exhibited narrow EL emission at a $\lambda_{\mathrm{EL}}$ of $446 \mathrm{~nm}(\mathrm{FWHM}=42 \mathrm{~nm})$ and $472 \mathrm{~nm}(\mathrm{FWHM}=46 \mathrm{~nm})$, respectively.

A red fluorophore, tetraphenyldibenzoperiflanthene (DBP, 1-28), was also synthesized by Bard et al.; this fluorophore had a narrow PL spectrum $\left(\lambda_{\mathrm{PL}}=596 \mathrm{~nm}\right.$, FWHM $=22 \mathrm{~nm}$ in benzene), and its solution-processed, TADF-sensitized red-emitting OLED (ITO/PEDOT:PSS/ 2 wt\% DBP:15 wt\% DC-TC:CBP/TmPyPB/LiF/Al) was successfully fabricated by Su, showing $\lambda_{\mathrm{EL}}=610 \mathrm{~nm}$ with a FWHM of $25 \mathrm{~nm}$, an EQE of 8\%, and a CIE coordinate of $(0.61,0.38)^{38,39}$. In 2020, Lee et al. reported an $N$-fused polyaromatic fluorophore (tDIDCz, 1-29) that exhibited violet emission at $393 \mathrm{~nm}$ in THF with a small FWHM $(20 \mathrm{~nm})^{40}$. In particular, the multiple resonance (MR) effect (discussed in detail in chapter 4) was achieved without any boron or ketone groups. The MR effect was generated by two nitrogen atoms enclosed in three aromatic rings with the LUMO distributed along the adjacent carbon atoms. The device employing tDIDCz showed a pure-violet EL emission at $\lambda_{\mathrm{EL}}=401 \mathrm{~nm}$ with a remarkably small FWHM (14 $\mathrm{nm})$.

\section{Quinacridone derivatives}

Various quinacridone derivatives based on a core structure consisting of five fused rings have proven to be green OLED emitters with great potential, showing intense fluorescence, high carrier mobility, and inherently narrow emission. However, the rigid and planar core structures readily form strong $\pi-\pi$ stacking and intermolecular hydrogen bonding interactions in concentrated or aggregated conditions, which significantly quenches their fluorescence. Wang et al. studied the light-emitting characteristics of several quinacridone derivatives with various substituents to reduce intermolecular aggregation. For example, the quinacridone derivatives $N, N$-di( $n$-butyl) quinacridone (1-30) and $N, N$-di( $n$-butyl)-1,3,8,10-tetramethylquinacridone (1-31) showed narrow PL spectra in chloroform with a PLQY of over $90 \%$ at $\lambda_{\mathrm{PL}}=538$ and 532 $\mathrm{nm}$, respectively $(\mathrm{FWHM}<30 \mathrm{~nm})^{41}$. EL devices fabricated with these molecules showed only a slight increase in the FWHM (28 and $36 \mathrm{~nm}$ for 1-30 and 1-31, respectively). Fluorine substituents were subsequently introduced to yield $N, N^{\prime}$-di(n-butyl)-2,9-difluoroquinacridone $\left(\mathrm{C}_{4} \text {-DFQA, 1-32 }\right)^{42}$. Similar to the non-fluorinated compounds 1-30, $\mathrm{C}_{4}$-DFQA showed a remarkably narrow $(\mathrm{FWHM}=21 \mathrm{~nm})$ and highly efficient green PL emission in THF at $535 \mathrm{~nm}$ with a PLQY of $97 \%$. The introduction of strongly electron-withdrawing fluorine atoms decreased the HOMO and LUMO energy levels, resulting in efficient electron injection and transport to improve the OLED performance. As shown in Fig. 4c, the EL spectra of the $\mathrm{ITO} / \mathrm{NPB} / \mathrm{C}_{4}-\mathrm{DFQA} / \mathrm{Alq}_{3} / \mathrm{LiF} / \mathrm{Al}$ device exhibited a 
yellowish-green emission at $553 \mathrm{~nm}$ with a small FWHM of $30 \mathrm{~nm}$ and CIE coordinates of $(0.42,0.56)$. The authors also attempted to extend the conjugation of the core to tune the emission properties of quinacridone derivatives ${ }^{43}$. The indole-fused quinacridone structure 5,8,15,18-tetraoctyl-5,8,15,18-tetrahydroindolo[3,2-a]indole $\left[3^{\prime}, 2^{\prime}: 5,6\right]$ quinacridone (IDQA, 1-33) in toluene showed a slightly redshifted PL maximum at $568 \mathrm{~nm}$ compared to that of quinacridone itself due to increased conjugation. In dilute solution, narrowband PL with an FWHM of $25 \mathrm{~nm}$ and a PLQY of 91\% was measured, while a redshifted broadened PL spectrum with decreased PLQY was observed in concentrated solutions due to aggregation. The ITO/NPB/ $\mathrm{Alq}_{3}$ :IDQA ( $\left.1 \mathrm{wt} \%\right) / \mathrm{Alq}_{3} / \mathrm{LiF} / \mathrm{Al}$ EL device exhibited a narrow emission at $\lambda_{\mathrm{EL}}=588 \mathrm{~nm}$ with an FWHM of $45 \mathrm{~nm}$.

\section{BODIPY derivatives}

BODIPY derivatives are efficient fluorescent materials with sharp absorption and emission spectra with high PLQY values close to unity. The emission wavelength of BODIPY derivatives can be modulated by functionalizing different positions with different substituents and/or controlling $\pi$-conjugation. For example, Baryshnikov et al. reported 1,7-diphenyl-substituted-BODIPY derivatives such as 2-carbethoxy-3-methyl-7-butyl-1,9-diphenyl-4,4difluoro-3a,4a,7-triaza-4-bora-cyclopenta[b]-fluoren-8-on $(1-34)^{44,45}$. Because of the unique nature of BODIPY structures, the geometrical distortion in the excited state is limited upon electronic transition between the HOMO and LUMO, resulting in a small Stokes shift. Compounds 1-34 showed a bright red emission in DCM with a $\lambda_{\mathrm{PL}}=$ $618 \mathrm{~nm}$, a PLQY of 86\%, a Stokes shift of $30 \mathrm{~nm}$, and an FWHM of $48 \mathrm{~nm}$. Narrow-emission spectra (FWHM of $45 \mathrm{~nm}$ ) were also obtained from EL devices (ITO/CuI/ CBP:20\% 1-34/Bphen/Ca/Al). A new phenyl amidesubstituted-BODIPY structure (1-35) was designed by Ziessel et al. ${ }^{46}$. Compounds 1-35 showed a PL maximum at $540 \mathrm{~nm}$ with an FWHM of $31 \mathrm{~nm}$ in DCM, while its PL spectra in film exhibited a strong secondary emission at $633 \mathrm{~nm}$ by forming dimers via hydrogen bonding. When the BODIPY-based emitters were dispersed into host materials $\left(\mathrm{Alq}_{3}\right)$, an EL device with the structure ITO/ $\alpha$ $\mathrm{NPD} / \mathrm{Alq}_{3}: 1-35 / \mathrm{BCP} / \mathrm{Al}$ achieved narrow EL emission. Interestingly, OLEDs doped with 7 and $18 \mathrm{~mol} \%$ of 1-35 showed a narrow EL emission with FWHM $<40 \mathrm{~nm}$ at $\lambda_{\mathrm{EL}}=550$ (monomer emission) and $635 \mathrm{~nm}$ (dimer emission), respectively, providing the opportunity to adjust the emission color from yellow to red without damaging the color purity (Fig. 4d, left). By attaching a naphthalene or pyrene substituent at the $\mathrm{C}-8$ position, Pushkarev et al. synthesized new BODIPY derivatives with extended $\pi$-conjugation, 4,4-difluoro-8-naphthyl-1,3,5,7tetramethyl-2,6-diethyl-4-boron-3a,4a-diaza-s-indacene
(1-36) and 4,4-difluoro-8-pyrryl-1,3,5,7-tetramethyl-2,6diethyl-4-boron-3a,4a-diaza-s-indacene $(1-37)^{47}$. Both BODIPY derivatives showed PL emission at 541-544 nm, regardless of the surrounding environment (DCM, ethanol, and poly(methyl methacrylate) (PMMA)). The ITO/ PVK: 1-36 or 1-37 (5 wt\%)/BPhen/ $/ \mathrm{Alq}_{3} / \mathrm{Yb}$ EL devices also showed narrow emission at $\lambda_{\mathrm{EL}}=542$ and $540 \mathrm{~nm}$, respectively, with an FWHM of $36 \mathrm{~nm}$. However, a significant excimer emission was also observed in the devices doped with $10 \mathrm{wt} \%$ BODIPY derivatives. To reduce fluorescence quenching via $\pi-\pi$ stacking, Ahn et al. designed a difluoroboron complex based on 2-(benzothiazol-2-yl)phenol (1-38) in which the boron is bound to $\mathrm{O}$ and $\mathrm{N}$ atoms in the bidentate ligand ${ }^{48}$. Compounds 1-38 showed a narrow and efficient PL emission at 463 $\mathrm{nm}$ with a PLQY of $61 \%$ and an FWHM of $38 \mathrm{~nm}$ even in the aggregated state, confirming that the $\pi-\pi$ stacking interactions were effectively suppressed. In addition, Zhan et al. demonstrated that the TADF-sensitized hyperfluorescence from 10-(4,4'-di-tert-butyl[1,1:3,1'-terphenyl]-5-yl)-5,5-difluoro-1,3,7,9-tetramethyl- $5 \mathrm{H}-4 \lambda^{4}, 5 \lambda^{4}$ -

dipyrrolo[1,2-c:2,1-f][1,3,2] diazaborinine (tPhBODIPY, 1-39) showed narrow EL spectra and a high $E Q E^{49}$. A hyperfluorescence device with $\mathrm{DCzDCN}$ as a host and 4CzIPN as a TADF assistant dopant displayed a bright green emission at an $\lambda_{\mathrm{EL}}$ of $520 \mathrm{~nm}$ (FWHM of $32 \mathrm{~nm}$ ) with $\mathrm{EQE}=19 \%$ and $\mathrm{CIE}$ coordinates of $(0.26,0.67)$ (Fig. 4d, right).

\section{Cyclometalated complex-based phosphorescent materials with narrow emission}

Cyclometalated complexes containing Ir, Pt, Pd, Eu, and $\mathrm{Tb}$ in their core surrounded by $\pi$-conjugated ligands show intense phosphorescence even at room temperature ${ }^{50,51}$. Through efficient ISC from $S_{1}$ to $T_{1}$, these phosphorescent emitters can achieve internal EL quantum efficiencies close to unity via strong spin-orbit coupling (SOC) between the emitting triplet state and high-lying singlet states, demonstrating their great potential as emitters in phosphorescent OLEDs (PhOLEDs) ${ }^{52-54}$.

Figure 5a shows a simplified molecular orbital (MO) diagram for organo-transition metal compounds ${ }^{55}$. The electrons in most cyclometalated complexes are populated in the ligand-centered (LC) and metal-to-ligand charge transfer (MLCT) states upon excitation, yielding mainly four excited electronic states of the singlet and triplet MLCT $\left({ }^{1} \mathrm{MLCT}\right.$ and $\left.{ }^{3} \mathrm{MLCT}\right)$ and LC $\left({ }^{1} \mathrm{LC}\right.$ and $\left.{ }^{3} \mathrm{LC}\right)$ states. Subsequently, the low-lying ${ }^{3} \mathrm{MLCT}$ and ${ }^{3} \mathrm{LC}$ states can be occupied by excited carriers via internal conversion (IC) and ISC. In many phosphorescent cyclometalated complexes, the lowest excited $T_{1}$ state can be described as a combination (or intermixing) of ${ }^{3}$ MLCT and ${ }^{3} \mathrm{LC}$, forming the LUMO of a hybrid triplet state. MLCT-dominant phosphorescence is common in 
a

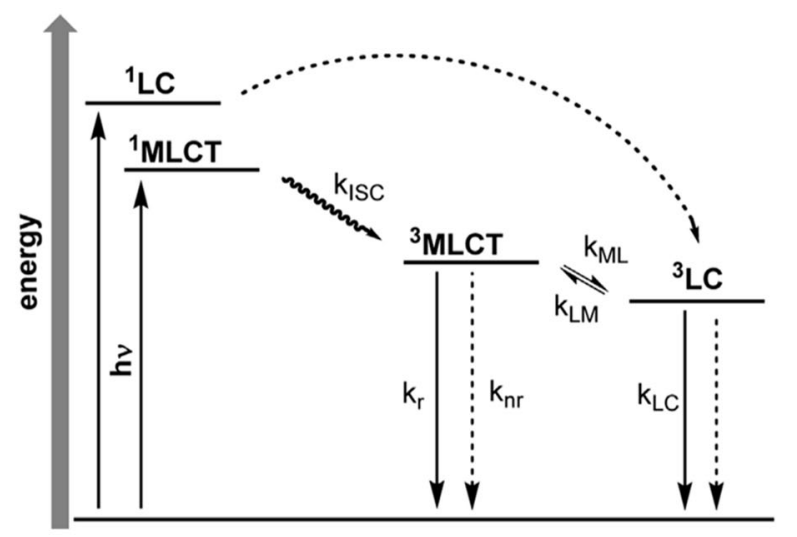

b

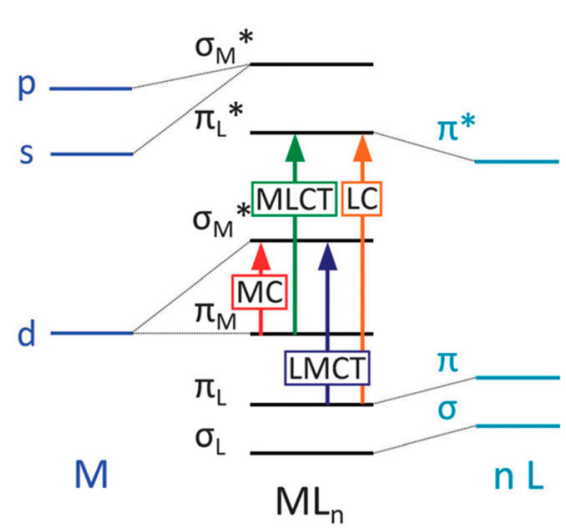

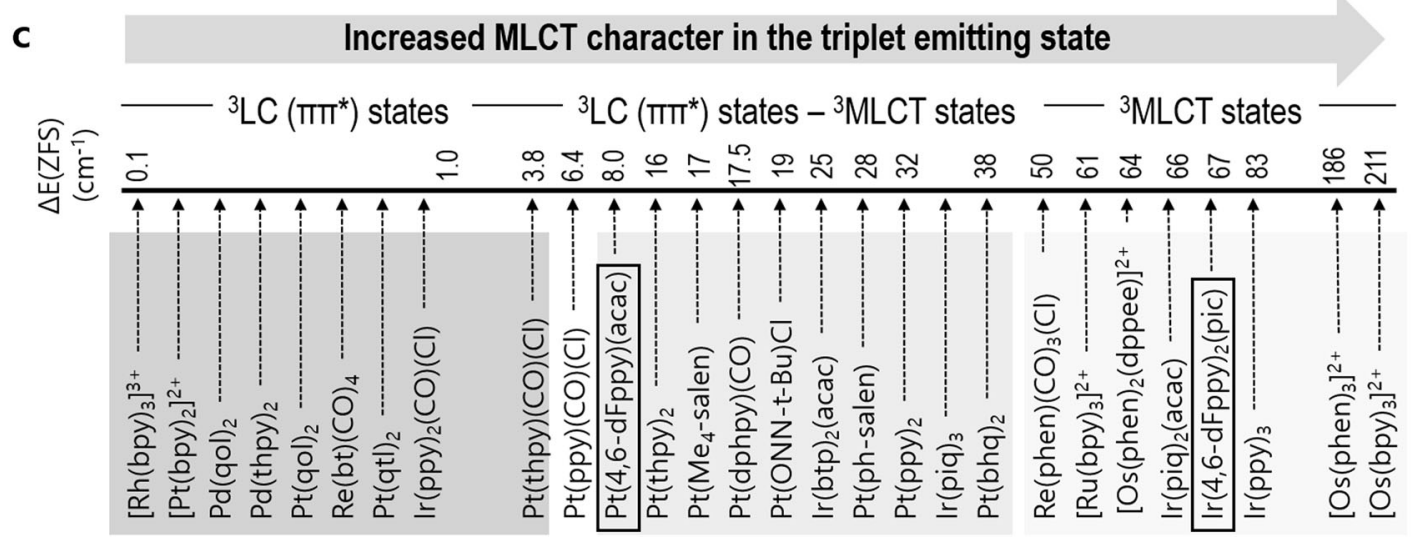

Fig. 5 Light-emitting characteristics of cyclometalated complexes. a Energy diagram of a cyclometalated complex. Reproduced with permission $^{55}$. Copyright 2015, Elsevier. b Possible electronic transitions in cyclometalated complexes ( $M=$ metal; $L=$ ligand). Reproduced with permission ${ }^{56}$. Copyright 2013, The Royal Society of Chemistry. c Correlation between MLCT character and ZFS in the $\mathrm{T}_{1}$ state.

phosphorescent Ir and Ru coordination complexes and is characterized by broad, structureless emission with pronounced solvatochromism and rigidochromism originating from strong CT characteristics. The MLCT-dominant emission in various Ir complexes has a relatively short phosphorescence lifetime ( $\tau_{\mathrm{p}}$ on the order of ns to $\mu \mathrm{s}$ ) because of the efficient SOC with a singlet excited state such as ${ }^{1}$ MLCT. By differentiating the central metal and organic ligands, cyclometalated complexes can also form various excited states, such as metal-centered (MC) and ligand-to-metal charge transfer (LMCT) states, as shown in Fig. $5 b^{56}$. LMCT is the charge transfer from an MO with ligand-like character to that with metal-like character, which is an opposite process of MLCT from an MO with metal-like character to that with ligand-like character. Thus, LMCT reduces the metal center, while MLCT undergoes oxidation of the metal center. Unlike homoleptic phosphorescent complexes with identical ligand structures, heteroleptic complexes with incorporated ancillary ligands $\left(\mathrm{L}^{\prime}\right)$ have additional transition states induced by the ligands, i.e., $\mathrm{L}^{\prime} \mathrm{C}, \mathrm{ML} \mathrm{C}^{\prime} \mathrm{C}$, and ligand to ligand charge transfer (LL'CT) states, facilitating fine modulation of phosphorescence color tuning.

The emitting $T_{1}$ state splits into three substates via zero-field splitting (ZFS) depending on the $\mathrm{SOC}^{54}$. The ZFS values correlate with the relative contribution of the ${ }^{3}$ MLCT and ${ }^{3} \mathrm{LC}$ states to the LUMO of cyclometalated complexes. As shown in Fig. 5c, the octahedral $\operatorname{Ir}(4,6-$ dFppy $)_{2}$ (pic) complex exhibits a larger ZFS and a shorter emission decay time $\left(\triangle \mathrm{E}(\mathrm{ZFS}, \mathrm{Ir})=67 \mathrm{~cm}^{-1}\right.$ and $\mathrm{t}(\mathrm{Ir})=$ $0.4 \mu \mathrm{s})$ compared to the square planar $\mathrm{Pt}(4,6-\mathrm{dFppy})(\mathrm{acac})$ complex $\left(\triangle E(\mathrm{ZFS}, \mathrm{Pt})=8 \mathrm{~cm}^{-1} \text { and } \mathrm{\tau}(\mathrm{Pt})=3.6 \mu \mathrm{s}\right)^{57}$. The emitting $\mathrm{T}_{1}$ state in $\mathrm{Pt}(4,6-\mathrm{dFppy})(\mathrm{acac})$ is largely $\mathrm{LC}$, showing a better resolvable emission compared to that of $\operatorname{Ir}(4,6-\mathrm{dFppy})_{2}$ (pic). Large $\Delta \mathrm{E}(\mathrm{ZFS})$ values over $50 \mathrm{~cm}^{-1}$ in quasi-octahedral structures indicate significant ${ }^{3} \mathrm{MLCT}$ character in the emitting $\mathrm{T}_{1}$ state, showing favorable radiative emission from the triplet substates to the ground $\mathrm{S}_{0}$ state via efficient $\mathrm{SOC}$. The smaller $\triangle \mathrm{E}(\mathrm{ZFS})$ from the emitting triplet state in Pt complexes originates from the weaker SOC between the lowest ${ }^{3} \mathrm{LC}\left({ }^{3} \pi \pi^{*}\right)$ and ${ }^{1} \mathrm{MLCT}$ $\left({ }^{1} \mathrm{~d} \pi^{*}\right)$ states. In this case, indirect SOC mixing can occur 
if the ${ }^{1}$ MLCT state interacts with the ${ }^{3}$ MLCT state, which couples with ${ }^{3} \mathrm{LC}$ by a configuration interaction $(\mathrm{CI})^{54}$. Compounds with $\triangle \mathrm{E}(\mathrm{ZFS})$ smaller than $1 \mathrm{~cm}^{-1}$ show favorable ${ }^{3} \mathrm{LC}$ emitting states. Cyclometalated complexes, which have a $T_{1}$ state with dominant ${ }^{3} \mathrm{MLCT}$ character, show a short phosphorescence lifetime with high quantum yield; however, structureless broad emissions are commonly observed, originating from the strong CT character of these states. To induce ${ }^{3} \mathrm{LC}$-dominant narrow phosphorescent emission, it is necessary to design phosphorescent molecules with a ${ }^{3} \mathrm{LC}$ state that is lower than the ${ }^{3}$ MLCT by modifying the structure of the organometallic complex. Vibrational coupling to the ground state in the ${ }^{3} \mathrm{LC}$-dominant emission can be effectively suppressed by employing rigid ligand frameworks without intra- or interligand CT interactions. In this chapter, we review cyclometalated complexes with narrow emission and discuss the correlation between the molecular structure and intermixing of the ${ }^{3} \mathrm{LC}$ and ${ }^{3} \mathrm{MLCT}$ states and the resulting light-emitting characteristics (Table 2).

\section{Ir-based phosphorescent cyclometalated complexes}

Since the Thompson and Forrest group developed fac-tris (2-phenylpyridine)iridium $\left(\mathrm{fac}-\operatorname{Ir}(\mathrm{ppy})_{3}\right)$ in the late $1990 \mathrm{~s}^{58}$, heavy metal-based phosphorescent emitters have been extensively studied with the aim of exploiting their strong SOC and triplet excitons. Ir(III) complexes have shown good photo- and thermal stabilities, high quantum efficiencies, and short lifetimes. For example, $f a c$ - $\operatorname{Ir}(\mathrm{ppy})_{3}$ is a representative green-emissive triplet emitter $\left(\lambda_{\mathrm{PL}}=519 \mathrm{~nm}\right)$ with a high PLQY of $\sim 100 \%$ at room temperature. However, it shows a broad emission spectrum due to its strong MLCT character at ambient temperature ${ }^{59}$. Therefore, cyclometalated Ir complexes with a variety of ligands have been synthesized to improve emission color purity by modulating the energy level and spatial geometry (Fig. 6). Bejoymohandas developed a series of Ir complexes (Ir1 (2-1), Ir2 (2-2), Ir3, and Ir4) by attaching electron-donating and electronwithdrawing substituents to the quinoline moiety in (benzo[b]thiophen-2-yl)quinoline cyclometalating ligands ${ }^{60}$. The HOMO and LUMO of Ir1-Ir4 are localized primarily in the cyclometalating ligands rather than the Ir center, resulting in an electronic transition with reduced MLCT character. As a result, the electron-donating methyl-substituted Ir1 and unsubstituted Ir2 showed narrow PL spectra at $655 \mathrm{~nm}$ in DCM with FWHM < 44 nm, while Ir3 and Ir4, which had strong electron-withdrawing trifluoromethyl and ethyl ester substituents, showed prominent CT character, resulting in a broad emission spectrum with a smaller emissive bandgap. The light-emitting properties of phosphorescent metal complexes can also be modulated by changing the ancillary ligand. Because of their higher triplet energy, nonchromophoric ancillary ligands do not influence the emission process directly, but different ligand field strengths can alter the SOC efficiency and structural distortion. Bejoymohandas developed new Ir complexes (Ir1-pic (2-3) and Ir2-pic (2-4)) by replacing the flexible thenoyltrifluoroacetylacetonate (tta) ancillary ligand of Ir1 and Ir2 (hereafter called Ir1-tta and Ir2-tta) with a rigid picolinate (pic) moiety ${ }^{61}$. As shown in Fig. $7 \mathrm{a}$, the change in ancillary ligands had a negligible influence on the shape of the PL spectrum and FWHM $(<44 \mathrm{~nm})$, but a slight blueshift in the PL spectra of the Ir1-pic and Ir2-pic was observed because of the stronger ligand field of the ancillary pic ligand compared to the tta moiety. The quantum efficiencies of Ir1-pic (49\%) and Ir2-pic (37\%) were significantly higher than their tta analogs $(2-8 \%)$ because the rigid picoline ligand reduces the extent of the geometrical deformation of the excited state and thereby suppresses nonradiative relaxation. With the device architecture ITO/ PEDOT:PSS/PVK/CBP:TPBi:Ir1-pic (15\%) or Ir2-pic (10\%)/ $\mathrm{TPBi} / \mathrm{LiF} / \mathrm{Al}, \mathrm{EQEs}$ of $5.03 \%$ and $3.41 \%$ were obtained for Ir1-pic and Ir2-pic-based devices with EL maxima at 657 and $664 \mathrm{~nm}$, respectively.

Huang reported a spiro aromatic ligand-based homoleptic Ir complex that demonstrated both narrow emission and color tunability ${ }^{62}$. Bulky 2-(spiro[fluorene-9,9'xanthene])-pyridine (SFXpy) acts as a multifunctional ligand. Its steric bulkiness not only reduces intermolecular $\pi-\pi$ aggregation at high doping levels but also helps charge injection/transport. Because of its extended conjugation, $f a c-\operatorname{Ir}(\mathrm{SFXpy})_{3}$ (2-5) showed a redshifted (compared to $f a c-\operatorname{Ir}(\mathrm{ppy})_{3}$ ) emission at $542 \mathrm{~nm}$, but no emission from the aggregates or excimers was observed (Fig. 7b). In addition, the fact that emission from the LC transition was predominant ensured a narrow PL emission with an FWHM of approximately $27 \mathrm{~nm}$. The PhOLED was fabricated with a common host (CBP) by varying the doping concentration of $f a c-\operatorname{Ir}(\mathrm{SFXpy})_{3}$ (5-18 wt\%) and showed narrow EL spectra at $\lambda_{\mathrm{EL}}=542 \mathrm{~nm}$ $(\mathrm{FWHM}=29 \mathrm{~nm})$ with a peak EQE of $12.1 \%$ in devices with a doping concentration of $15 \mathrm{wt} \%$. Using combinatorial chemistry methods, König et al. studied the photophysical properties of a series of Ir complexes (2-6 29) and was able to achieve narrow PL (FWHM $<49 \mathrm{~nm}$ ) and PLQY ranging from 54\%-85\% through modification of the ligand structure ${ }^{63}$. Hwang reported a solutionprocessable Ir complex, bis(2-(4-methoxynaphthalen-1yl)benzo[d]thiazole)iridium(III)acetylacetonate

$\left((\mathrm{BTMN})_{2} \operatorname{Ir}(\mathrm{acac}), 2-10\right)^{64}$. In DCM solution, $(\mathrm{BTMN})_{2} \operatorname{Ir}$ (acac) had a remarkably narrow PL spectrum with an FWHM of $\sim 25 \mathrm{~nm}$ at $\lambda_{\mathrm{PL}}=599 \mathrm{~nm}$ (PLQY of 29\%). However, the $(\mathrm{BTMN})_{2} \operatorname{Ir}(\mathrm{acac})$-based red-emissive EL device (ITO/PEDOT:PSS/PVK:(BTMN) $)_{2} \operatorname{Ir}(\mathrm{acac}) / \mathrm{TPBi} /$ $\mathrm{CsF} / \mathrm{Al}$ ) showed a significantly broadened emission at 598 $\mathrm{nm}$ with an FWHM in the range of 74-76 nm, reinforcing the need for additional structural modifications to narrow the EL bandwidth to achieve high color purity. 
Table 2 Summary of optical and EL device properties of organometallic complexes.

\begin{tabular}{|c|c|c|c|c|c|c|c|c|c|}
\hline No. & Emitter & $\lambda_{\mathrm{PL}}(\mathrm{nm})$ & FWHM $_{\mathrm{PL}}(\mathrm{nm})$ & PLQY (\%) & $\lambda_{\mathrm{EL}}(\mathrm{nm})$ & $\mathrm{FWHM}_{\mathrm{EL}}(\mathrm{nm})$ & $\mathrm{EQE}(\%)$ & $\mathrm{ClE}_{x, y}$ & Refs. \\
\hline \multicolumn{10}{|c|}{ Ir complexes (octahedral structure) } \\
\hline $2-1$ & $\mid r 1^{a}$ & $\begin{array}{l}655 \text { (in DCM and } \\
\text { PMMA film) }\end{array}$ & $\begin{array}{l}42^{*} \text { (in DCM and } \\
\text { PMMA film) }\end{array}$ & $\begin{array}{l}2 \text { (in DCM) } \\
24 \text { (in } \\
\text { PMMA film) }\end{array}$ & 657 & $43^{*}$ & 2.05 & $(0.71,0.28)$ & 60 \\
\hline $2-2$ & $\operatorname{lr} 2^{\mathrm{a}}$ & $\begin{array}{l}665 \text { (in DCM and } \\
\text { PMMA film) }\end{array}$ & $\begin{array}{l}44^{*} \text { (in DCM and } \\
\text { PMMA film) }\end{array}$ & $\begin{array}{l}8 \text { (in DCM) } \\
31 \text { (in } \\
\text { PMMA film) }\end{array}$ & 667 & $46^{*}$ & 2.11 & $(0.71,0.28)$ & \\
\hline $2-3$ & Ir1-pic ${ }^{b}$ & $\begin{array}{l}651 \text { (in DCM and } \\
\text { PMMA film) }\end{array}$ & $\begin{array}{l}43^{*} \text { (in DCM) } \\
42^{*} \text { (in PMMA film) }\end{array}$ & $\begin{array}{l}49 \text { (in DCM) } \\
49 \text { (in } \\
\text { PMMA film) }\end{array}$ & 657 & $48^{*}$ & 5.04 & $(0.72,0.28)$ & 61 \\
\hline $2-4$ & Ir2-pic ${ }^{c}$ & $\begin{array}{l}660 \text { (in DCM and } \\
\text { PMMA film) }\end{array}$ & $\begin{array}{l}44^{*} \text { (in DCM) } \\
42^{*} \text { (in PMMA film) }\end{array}$ & $\begin{array}{l}37 \text { (in DCM) } \\
39 \text { (in } \\
\text { PMMA film) }\end{array}$ & 664 & $48^{*}$ & 3.67 & $(0.72,0.28)$ & \\
\hline $2-5$ & $\mathrm{Fac}-\operatorname{Ir}(\mathrm{SFXpy})_{3}{ }^{\mathrm{d}}$ & 542 (in DCM) & $27^{*}$ (in DCM) & 18 (in DCM) & 542 & $29^{*}$ & 12.1 & $(0.42,0.58)$ & 62 \\
\hline $2-6$ & NNN & 520 & 46 & 85 (in DCM) & NA & NA & NA & NA & 63 \\
\hline $2-7$ & NNO & 522 & 45 & 77 (in DCM) & NA & NA & NA & NA & \\
\hline $2-8$ & NNP & 518 & 47 & 83 (in DCM) & NA & NA & NA & NA & \\
\hline $2-9$ & NPP & 515 & 44 & 76 (in DCM) & NA & NA & NA & NA & \\
\hline $2-10$ & $(B T M N)_{2} \operatorname{Ir}(a c a c)^{e}$ & 599 (in DCM) & $25^{*}$ (in DCM) & 29 (in DCM) & 598 & 74 & 10.8 & $(0.59,0.37)^{f}$ & 64 \\
\hline \multicolumn{10}{|c|}{ Pt and Pd complexes (square planar structures) } \\
\hline $3-1$ & PtON1-Me & $\begin{array}{l}444 \text { (in DCM) } \\
445 \text { (in PMMA film) }\end{array}$ & 20 (in DCM) & $\begin{array}{l}89 \text { (in DCM) } \\
84 \text { (in } \\
\text { PMMA film) }\end{array}$ & NA & NA & NA & NA & 66 \\
\hline $3-2$ & PtON1-tBu ${ }^{9}$ & $\begin{array}{l}444 \text { (in DCM) } \\
445 \text { (in PMMA film) }\end{array}$ & 20 (in DCM) & $\begin{array}{l}95 \text { (in DCM) } \\
88 \text { (in } \\
\text { PMMA film) }\end{array}$ & 448 & 24 & 5.3 & $(0.151,0.098)$ & 65,66 \\
\hline $3-3$ & PtON1-NMe 2 & $\begin{array}{l}442 \text { (in DCM) } \\
440 \text { (in PMMA film) }\end{array}$ & 15 (in DCM) & $\begin{array}{l}80 \text { (in DCM) } \\
88 \text { (in } \\
\text { PMMA film) }\end{array}$ & NA & NA & NA & NA & 66 \\
\hline $3-4$ & PtON6-tBu ${ }^{9}$ & $\begin{array}{l}448 \text { (in DCM) } \\
447 \text { (in PMMA film) }\end{array}$ & 20 (in DCM) & $\begin{array}{l}81 \text { (in } \\
\text { PMMA film) }\end{array}$ & 452 & 30 & 10.9 & $(0.147,0.093)$ & 65,66 \\
\hline $3-5$ & PtON7-dtb ${ }^{h}$ & $\begin{array}{l}446 \text { (in DCM) } \\
447 \text { (in PMMA film) }\end{array}$ & 20 (in DCM) & $\begin{array}{l}85 \text { (in DCM) } \\
91 \text { (in } \\
\text { PMMA film) }\end{array}$ & 451 & 29 & 24.8 & $(0.148,0.079)$ & \\
\hline $3-6$ & PtN1Ni & 491 (in DCM) & 18 (in DCM) & 81 (in DCM) & 498 & 20 & 26.1 & $(0.15,0.56)$ & 68 \\
\hline $3-7$ & PtN1pyCl & 482 (in DCM) & 18 (in DCM) & 3 (in DCM) & NA & NA & NA & NA & \\
\hline $3-8$ & PtON7-tBu & 446 (in DCM) & 20 (in DCM) & 83 (in DCM) & 450 & 28 & 17.6 & $(0.14,0.09)$ & \\
\hline $3-9$ & PtN8ppy ${ }^{k}$ & 573 (in DCM) & 26 (in DCM) & 40 (in DCM) & 576 & 28 & 19.3 & $(0.53,0.47)$ & \\
\hline $3-10$ & Pt(ppzOczpy) & $\begin{array}{l}453 \text { (in DCM) } \\
449 \text { (in PMMA film) }\end{array}$ & $\begin{array}{l}69 \text { (in DCM) } \\
22 \text { (in PMMA film) }\end{array}$ & $\begin{array}{l}39 \text { (in DCM) } \\
53 \text { (in } \\
\text { PMMA film) }\end{array}$ & NA & NA & NA & NA & 69 \\
\hline $3-11$ & Pt(ppzOczpy-m) & $\begin{array}{l}450 \text { (in DCM) } \\
447 \text { (in PMMA film) }\end{array}$ & $\begin{array}{l}29 \text { (in DCM) } \\
19 \text { (in PMMA film) }\end{array}$ & 86 (in DCM) & NA & NA & NA & NA & \\
\hline $3-12$ & Pt(ppzOczpy-2m) & $\begin{array}{l}450 \text { (in DCM) } \\
448 \text { (in PMMA film) }\end{array}$ & $\begin{array}{l}28 \text { (in DCM) } \\
19 \text { (in PMMA film) }\end{array}$ & $\begin{array}{l}87 \text { (in DCM) } \\
67 \text { (in } \\
\text { PMMA film) }\end{array}$ & NA & NA & NA & NA & \\
\hline $3-13$ & Pt(ppzOczpy-4m) & $\begin{array}{l}449 \text { (in DCM) } \\
448 \text { (in PMMA film) }\end{array}$ & $\begin{array}{l}25 \text { (in DCM) } \\
22 \text { (in PMMA film) }\end{array}$ & $\begin{array}{l}92 \text { (in DCM) } \\
6 \text { (in PMMA film) }\end{array}$ & 450 & 53 & 19.5 & $(0.159,0.166)$ & \\
\hline $3-14$ & PtOEP ${ }^{m}$ & NA & NA & 50 (in PS film) & 650 & $19^{*}$ & 4 & NA & 70 \\
\hline $3-15$ & $\mathrm{PtOX}^{\mathrm{m}}$ & 648 (in PS film) & 26 (in PS film) & 44 (in Tol:DMF) & 650 & $25^{*, n}$ & 1.5 & $(0.69,0.30)^{n}$ & 71 \\
\hline $3-16$ & PtDPP ${ }^{m}$ & 630 (in PS film) & $33^{*}$ (in PS film) & 16 (in Tol:DMF) & 640 & $50^{*, n}$ & 0.25 & $(0.67,0.31)^{n}$ & \\
\hline $3-17$ & (Phpy)Pd(Pery-q) & $\begin{array}{l}635 \text { (in DCM) } \\
625 \text { (in PVK film) }\end{array}$ & $\begin{array}{l}27^{*} \text { (in DCM) } \\
19^{*} \text { (in PVK film) }\end{array}$ & NA & NA & NA & NA & NA & 73 \\
\hline $3-18$ & PdN1N & 472 (in DCM) & $18^{*}$ (in DCM) & 70 (in DCM) & NA & NA & NA & NA & 74 \\
\hline $3-19$ & PdN1N-dm & 470 (in DCM) & $22^{*}$ (in DCM) & 77 (in DCM) & 476 & $25^{*}$ & 25.1 & $(0.14,0.25)$ & \\
\hline $3-20$ & PdN6N & 476 (in DCM) & $18^{*}$ (in DCM) & 59 (in DCM) & NA & NA & NA & NA & \\
\hline $3-21$ & PdNTBP & 875 (in Tol) & $32^{*}$ (in Tol) & 8 (in Tol) & NA & NA & NA & NA & 75 \\
\hline $3-22$ & PdTPTBP & 800 (in Tol) & $47^{*}$ (in Tol) & 21 (in Tol) & NA & NA & NA & NA & \\
\hline $3-23$ & $\mathrm{Pd} 2 \mathrm{NF}$ & 868 (in Tol) & $41^{*}$ (in Tol) & 12 (in Tol) & NA & NA & NA & NA & 76 \\
\hline
\end{tabular}

Eu and $\mathrm{Tb}$ complexes 
Table 2 continued

\begin{tabular}{|c|c|c|c|c|c|c|c|c|c|}
\hline No. & Emitter & $\lambda_{\mathrm{PL}}(\mathrm{nm})$ & $\mathrm{FWHM}_{\mathrm{PL}}(\mathrm{nm})$ & PLQY (\%) & $\lambda_{\mathrm{EL}}(\mathrm{nm})$ & $\mathrm{FWHM}_{\mathrm{EL}}(\mathrm{nm})$ & EQE(\%) & $\mathrm{ClE}_{x, y}$ & Refs. \\
\hline $4-1$ & $\mathrm{Eu}(\mathrm{cbtfa})_{3}(\mathrm{bath})^{\mathrm{p}}$ & $\begin{array}{l}613 \text { (in CF, powder } \\
\text { and film) }\end{array}$ & $\begin{array}{l}4^{*} \text { (in powder) } \\
5^{*} \text { (in CF) } \\
9^{*} \text { (in film) }\end{array}$ & $\begin{array}{l}60 \text { (in powder) } \\
50 \text { (in film) }\end{array}$ & 613 & $5^{*}$ & $N A(<1)$ & $(0.63,0.34)$ & 84 \\
\hline $4-2$ & Eu(TTA $)_{3}$ Phen-FI-TPA & 612 (in CF and film) & $\begin{array}{l}5^{*} \text { (in CF) } 10^{*} \\
\text { (in film) }\end{array}$ & 34.1 (in CF) & NA & NA & NA & $(0.62,0.36)$ & 85 \\
\hline $4-3$ & $\mathrm{Eu}(\mathrm{HPhN})_{3}$ phen & 611 (in PS film) & $5^{*}$ (in PS film) & 18 (in PS film) & NA & NA & NA & NA & 86 \\
\hline $4-4$ & $\mathrm{Eu}(\mathrm{HPhN})_{3} \mathrm{dpp}$ & 611 (in PS film) & $5^{*}$ (in PS film) & 18 (in PS film) & NA & NA & NA & NA & \\
\hline $4-5$ & $\mathrm{Eu}(\mathrm{HPhN})_{3} \mathrm{DDXPO}$ & 611 (in PS film) & $5^{*}$ (in PS film) & 20 (in PS film) & NA & NA & NA & NA & \\
\hline $4-6$ & $\begin{array}{l}\mathrm{Eu}(\mathrm{DBM})_{3} \text { Phen-pCN- } \\
\mathrm{mCF}_{3}\end{array}$ & 612 (in CF) & 8.66 (in CF) & 30 (in film) & NA & NA & NA & NA & 87 \\
\hline $4-7$ & $\begin{array}{l}\mathrm{Eu}(\mathrm{DBM})_{3} \text { Phen-pCN- } \\
\mathrm{pCF}_{3}\end{array}$ & 612 (in CF) & 4.55 (in CF) & 24.1 (in film) & NA & NA & NA & NA & \\
\hline $4-8$ & $\begin{array}{l}\mathrm{Eu}(\mathrm{DBM})_{3} \text { Phen-pCN- } \\
\mathrm{pCH}_{3}\end{array}$ & 612 (in CF) & 8.61 (in CF) & 70.1 (in film) & NA & NA & NA & NA & \\
\hline 4-9 & $\begin{array}{l}\text { Eu(DBM) })_{3} \text { Phen-pCN- } \\
1 \text { Naph }\end{array}$ & 612 (in CF) & 4.33 (in CF) & 35.8 (in film) & NA & NA & NA & NA & \\
\hline $4-10$ & $\begin{array}{l}\text { Eu(DBM) })_{3} \text { Phen-pCN- } \\
2 \mathrm{Naph}\end{array}$ & 612 (in CF) & 4.34 (in CF) & 14.1 (in film) & NA & NA & NA & NA & \\
\hline $4-11$ & $\mathrm{~Tb}(\mathrm{PMIP})_{3} \mathrm{DPPOC}^{\mathrm{a}}$ & $\begin{array}{l}548 \text { (in DCM) } \\
542 \text { (in film) }\end{array}$ & $13^{*}$ (in DCM) & 16.7 (in DCM) & 548 & $13^{*}$ & NA & NA & 89 \\
\hline $4-12$ & $\mathrm{~Tb}(\mathrm{PMIP})_{3}\left(\mathrm{H}_{2} \mathrm{O}\right)_{2}$ & $\begin{array}{l}547 \text { (in DCM) } \\
543 \text { (in film) }\end{array}$ & $13^{*}$ (in DCM) & 10.4 (in DCM) & NA & NA & NA & NA & \\
\hline $4-13$ & $\mathrm{~Tb}(\mathrm{PMIP})_{3}(\mathrm{TPPO})_{2}$ & $\begin{array}{l}548 \text { (in DCM) } \\
543 \text { (in film) }\end{array}$ & 13* (in DCM) & 14.6 (in DCM) & NA & NA & NA & NA & \\
\hline $4-14$ & $\mathrm{~Tb}(\mathrm{tfac})_{3} \mathrm{DPEPO}$ & $\begin{array}{l}545 \text { (in DCM } \\
\text { and film) }\end{array}$ & $\begin{array}{l}20^{*} \text { (in DCM } \\
\text { and film) }\end{array}$ & $\begin{array}{l}48 \text { (in DCM) } \\
53 \text { (in film) }\end{array}$ & NA & NA & NA & NA & 90 \\
\hline
\end{tabular}

PS polystyrene, DMF dimethylformamide, Liq 8-quinolinolato lithium, m-MTDATA 4,4',4"'tris[(3-methylphenyl)phenylamino]triphenylamine, Libpp lithium 2-(2',2"bipyridine-6'-yl)phenolate, 26mCPy 2,6-bis(N-carbazolyl) pyridine, DPPS diphenyl-bis[4-(pyridin-3-yl)phenyl]-silane, PO15 2,8-bis(diphenylphosphoryl)-dibenzothiophene, CzSi 9-(4-tert-butylphenyl)-3,6-bis(triphenylsilyl)-9H-carbazole, CzAcSF 10-((4-((4-9H-carbazol-9-yl)phenyl)-sulfonyl)-phenyl)-9,9-dimethyl-9,10-dihydroacridine, DPEPO bis[2-(diphenylphosphino)-phenyl]ether oxide.

*Estimated values based on the graph (not provided by author).

aTO/PEDOT:PSS/mCP:TCTA:emitters/TPBi/Liq/Al.

b/TO/PEDOT:PSS/PVK/CBP:TPBi:emitter (15\%)/TPBi/LiF/AI.

CITO/PEDOT:PSS/PVK/CBP:TPBi:emitter (10\%)/TPBi/LiF/AI.

dITO/MoOx/m-MTDATA/Ir(ppz)3/CBP:emitter (15 wt\%)/Bphen/Libpp/Al.

ITO/PEDOT:PSS/PVK:emitter (7\%)/TPBi/CsF/AI.

fObtained at a luminance of $1000 \mathrm{~cd} \mathrm{~cm}^{-2}$.

ITO/HATCN/NPD/TAPC/2\% emitter:26mCPy/DPPS/BmPyPB/LiF/Al.

hITO/HATCN/NPD/TAPC/6\% emitter:47\% TAPC:47\% PO15/PO15/BmPyPB/LiF/Al.

IITO/PEDOT:PSS/NPD/TAPC/7\% emitter:26mCPy/PO15/BmPyPB/LiF/Al.

$\mathrm{j}$ ITO/HATCN/NPD/TAPC/6\% emitter:26mCPy/DPPS/BmPyPB/LiF/Al.

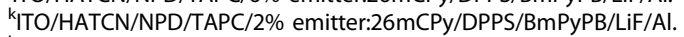

ITO/PEDOT:PSS/CzSi:CzAcSF:emitter (80:20:20)/DPEPO/TmPyPB/Liq/Al.

mITO/a-NPD/Alq3:6\% emitter/Alq3/Mg:Ag/Ag.

nobtained at a current density of $2 \mathrm{~mA} \mathrm{~cm}^{-2}$.

ITO/HATCN/NPD/TAPC/10\% emitter:26mCPy/DPPS/BmPyPB/LiF/AI

PITO/PEDOT:PSS/emitter/Ca/Al.

${ }^{\mathrm{q}} \mathrm{ITO} / \mathrm{NPB} / \mathrm{emitter} / \mathrm{BCP} / \mathrm{Alq} 3 / \mathrm{Mg} 0.9 \mathrm{Ag} 0.1 / \mathrm{Ag}$.

\section{Pt- and Pd-based phosphorescent cyclometalated complexes}

The $\mathrm{T}_{1}$ state of most octahedral Ir complexes is primarily determined by the ${ }^{3}$ MLCT state, which induces broad PL emission with high PLQY because of the strong SOC between the triplet and singlet states. In contrast, square planar $\mathrm{Pt}$ and $\mathrm{Pd}$ complexes typically have narrowband emission, but longer phosphorescence lifetimes and lower PLQYs than those found in Ir complexes were observed because of weak SOC in the square planar complexes (Fig. 8). Li et al. modified the ligand structure of the PtON1 structure that had a bridging oxygen between the phenyl-pyrazole (ppz)-based cyclometalating ligand and the carbazolyl pyridine ancillary ligand by incorporating electron-donating substituents (methyl (PtON1-Me, 3-1), tert-butyl (PtON1-tBu, 3-2), and N,Ndimethylamino (PtON1-NMe, $3-3)$ ) at the 4-position of the pyridyl ring, resulting in narrow deep-blue emissions (FWHM 15-20 nm) at $445 \mathrm{~nm}$ with high PLQYs (80 95\%) (Fig. 9) ${ }^{65,66}$. These results can be explained by the fact that the ${ }^{1} \mathrm{MLCT} /{ }^{3} \mathrm{MLCT}$ character in the original PtON1 was thermally accessible at room temperature, while the addition of an electron-donating group increased the energy levels of MLCT states, suppressing 


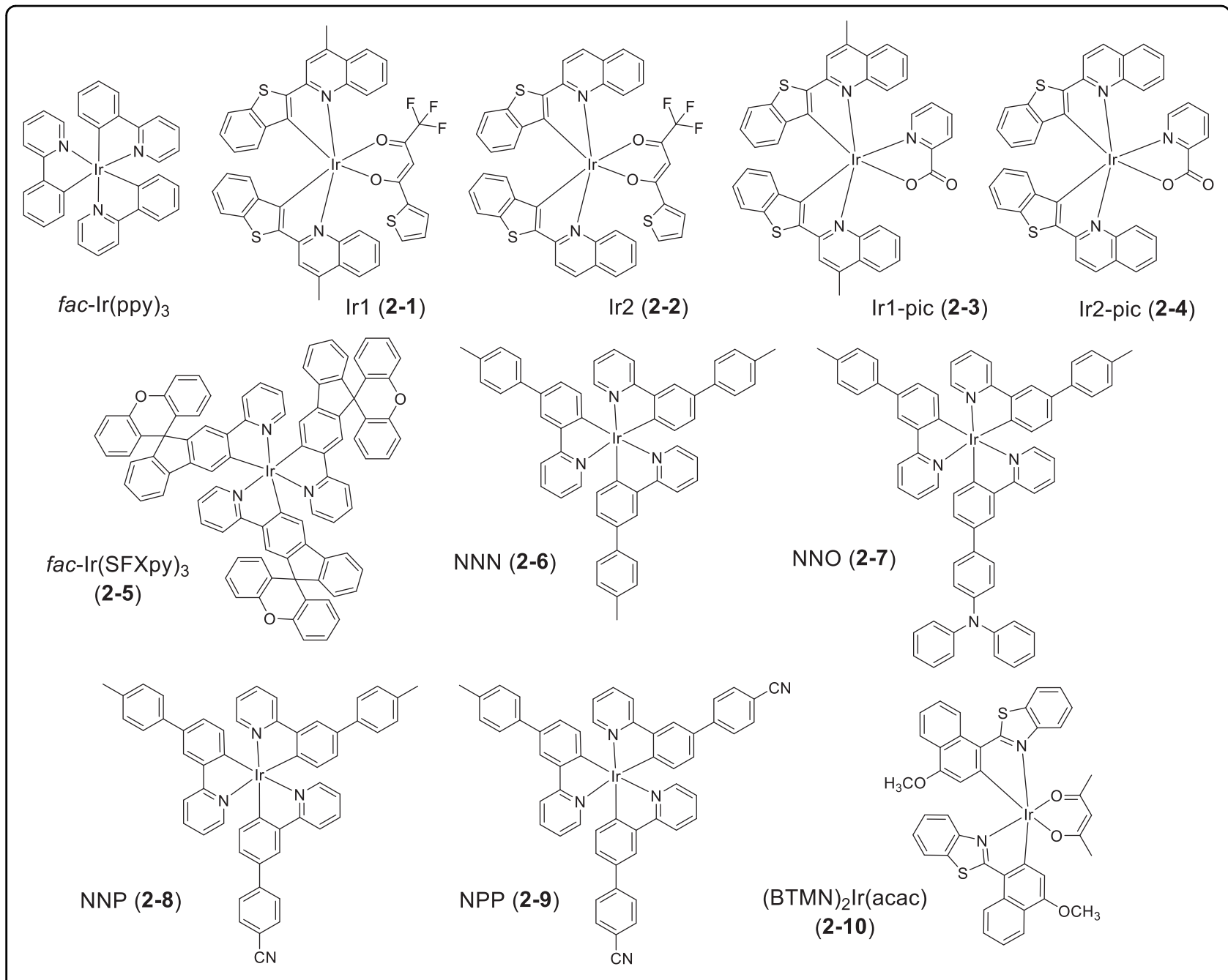

Fig. 6 Chemical structures of Ir complexes.

the MLCT character in the $\mathrm{T}_{1}$ state $\mathrm{s}^{51,67}$. In contrast, the addition of a 9-carbazolyl substituent (PtON1-Cz) caused broad CT emission due to the decreased MLCT energy level, which enhanced the MLCT character of the $\mathrm{T}_{1}$ state. A similar strategy was successfully employed with 4phenyl-pyrazole and carbene-based tetradentate Pt complexes (PtON6-tBu (3-4), PtON7-tBu, and PtON7-dtb (3-5)) by incorporating a tert-butyl substituent, resulting in an FWHM of 19-20 nm and a PLQY > 80\%. Li et al. also developed PtN1N (3-6), which included a pyrazolylcarbazole group as the emitting ligand and a pyridylcarbazole moiety as an ancillary ligand ${ }^{68}$. At $77 \mathrm{~K}$, the PL spectra of PtN1N and its analogs (PtN1pyCl (3-7) and PtN1(acac)) were similar, with each having a dominant single emission. At room temperature, PtN1N still showed little color contamination from $v_{0-1}$ and $v_{0-2}$ transitions $(S=0.3)$ with FWHM $<20 \mathrm{~nm}$, while PtN1pyCl had an $S$ value $>0.5$ and PtN1(acac) exhibited a structureless broad emission, whose appearance was dependent on the relative MLCT:LC character of the $\mathrm{T}_{1}$ state. Most importantly, a narrow EL emission (FWHM $20 \mathrm{~nm}$ ) and an EQE of $26.1 \%$ were obtained from a device with a structure of ITO/PEDOT:PSS/NPD/TAPC/ 7\% PtN1N:26mCPy/PO15/BmPyPB/LiF/Al. Because the excimer was absent due to the breaking of its planar geometry, a high EQE (25.6\%) was observed even with a high dopant concentration (14\%). Additionally, the blueemissive PtON7-tBu (3-8) and orange-emissive PtN8ppy (3-9) showed narrow PL spectra in DCM at $446 \mathrm{~nm}$ with FWHM $=20 \mathrm{~nm}$ (PLQY = 83\%) and $573 \mathrm{~nm}$ with FWHM $=26 \mathrm{~nm}$ (PLQY $=40 \%)$, respectively. By modifying the number and position of methyl substituents, a series of narrow-emissive $\mathrm{Pt}$ complexes, $\mathrm{Pt}(\mathrm{ppzOczpy})$ (3-10), Pt(ppzOczpy-m) (3-11), Pt(ppzOczpy-2m) (312), and $\mathrm{Pt}(\mathrm{ppzOczpy}-4 \mathrm{~m})$ (3-13), were synthesized by Huang et al., in which the intermixing of ${ }^{3} \mathrm{MLCT}$ and ${ }^{3} \mathrm{LC}$ 


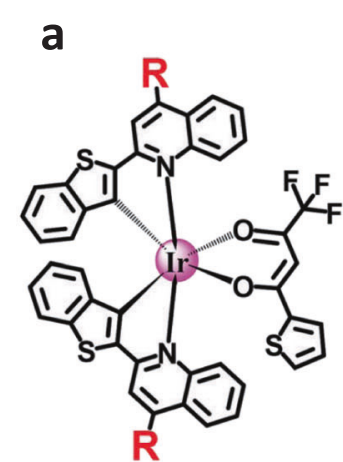

Ir1-tta; $\mathbf{R}=-\mathrm{CH}_{3}$ Ir2-tta; $\mathbf{R}=-\mathbf{H}$

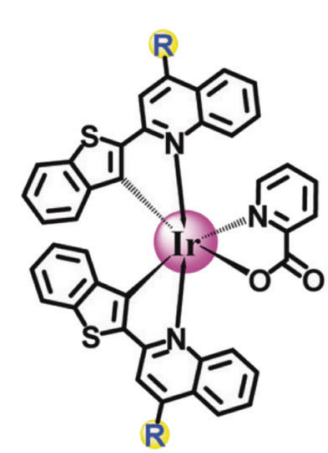

Ir1-pic; $\mathrm{R}=-\mathrm{CH}_{3} \quad 0$ Ir2-pic; $\mathbf{R}=-\mathbf{H}$

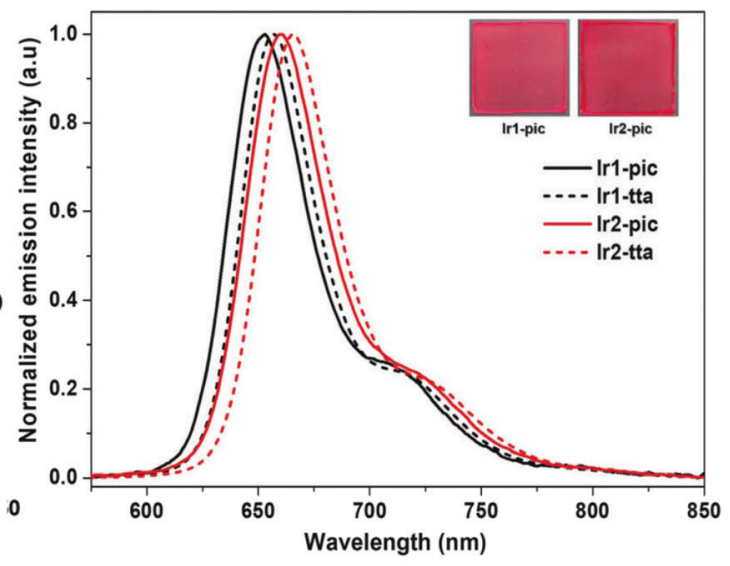

b

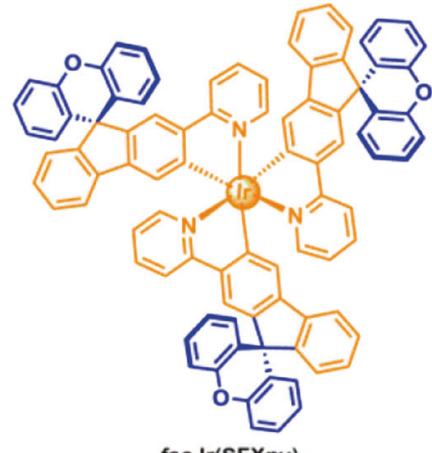

fac-Ir(SFXp) $)_{3}$

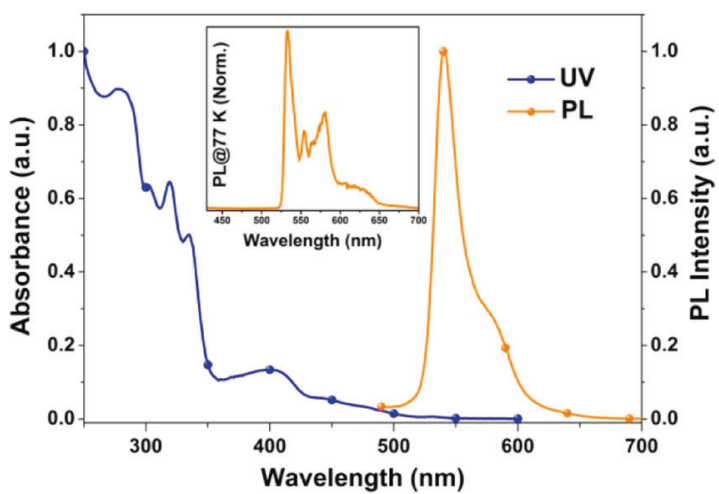

Fig. 7 Optical properties of Ir-containing cyclometalated complexes. Chemical structures and optical properties of a Ir1-tta, Ir2-tta, Ir 1-pic, and Ir2-pic in PMMA polymer films with 5 wt\% doping and $\mathbf{b}$ fac-Ir(SFXpy) ${ }_{3}$ in DCM solution. a Reproduced with permission ${ }^{61}$. Copyright 2019, The Royal Society of Chemistry. b Reproduced with permission ${ }^{62}$. Copyright 2017, American Chemical Society.

in the $\mathrm{T}_{1}$ state could be finely modulated ${ }^{69}$. Compared to a PtON1 reference material, the introduction of a methyl group to the para-position of the pyridine moieties enhances the electron distribution in the carbazole moieties to yield a ${ }^{3} \pi \pi^{*}$ dominated $\mathrm{T}_{1}$ state, resulting in a dominant $\mathrm{LC}$ contribution. Consequently, all four compounds had narrow PL spectra with remarkably low FWHM values of approximately $20 \mathrm{~nm}$ in PMMA films. PhOLEDs doped with 20\% Pt(ppzOczpy$4 \mathrm{~m})$ in the device with a structure of ITO/PEDOT:PSS/CzSi: CzAcSF:Pt(ppzOczpy-4m) (80:20:20)/DPEPO/TmPyPB/Liq/ $\mathrm{Al}$ showed the best EQE of $19.5 \%$ with CIE coordinates of $(0.159,0.166)$ and a FWHM of $53 \mathrm{~nm}$.

Pt complexes with porphyrin ligands were also studied as narrowband phosphorescent emitters. In 1998, Forrest et al. first reported the utilization of Pt-porphyrin complexes in efficient PhOLEDs by doping the host $\mathrm{Alq}_{3}$ with 2,3,7,8,12,13,17,18-octaethyl-21H,23H-porphine platinum (II) (PtOEP, 3-14) ${ }^{70}$. Although porphyrin is known to have a long-lived triplet state, PtOEP showed a decreased phosphorescence lifetime (91 $\mu \mathrm{s})$ and 50\% PLQY due to strong SOC. The Alq 3 :PtOEP-based PhOLED device has an emission spectrum similar to that of its porphyrin ligand and exhibits strong phosphorescence at $650 \mathrm{~nm}$ with a small FWHM of $19 \mathrm{~nm}$, which is thought to originate from the dominant LC character of the emitting $T_{1}$ state. Thompson also reported another type of Pt-porphyrin complex; the two representative examples, PtOX (3-15) and PtDPP (3-16) had octaalkylporphyrin and arylporphyrin ligands, respectively ${ }^{71}$. PtOX showed a narrow emission peak at $648 \mathrm{~nm}$ with FWHM $=26 \mathrm{~nm}$ in polystyrene films, and PtDPP also displayed a narrow PL spectrum at $\lambda_{\mathrm{PL}}=630 \mathrm{~nm}(\mathrm{FWHM}=33 \mathrm{~nm})$. The rotation of the phenyl group in PtDPP promoted nonradiative relaxation, making the PLQY of PtDPP (16\%) lower than that of PtOX (44\%). However, the spin-coated PtDPP:PS thin film showed an improved PLQY similar to that of PtOX because the rotation of the phenyl group was restricted. Narrow EL spectra of ITO/ $\alpha-\mathrm{NPD} / \mathrm{Alq}_{3}: \mathrm{PtOX}$ and PtDPP $/ \mathrm{Alq}_{3} / \mathrm{Mg}: \mathrm{Ag} / \mathrm{Ag}$ were observed at $\lambda_{\mathrm{EL}}=650 \mathrm{~nm}$ (FWHM of $25 \mathrm{~nm}$ ) and $640 \mathrm{~nm}$ (FWHM of $50 \mathrm{~nm}$ ) with CIE coordinates of $(0.69,0.30)$ and $(0.67,0.31)$, respectively. This result demonstrates the potential utility of PtOX- and PtDPP-based PhOLEDs as red emitters with satisfactory CIE criteria $(0.55 \leq x \leq 0.74$ and $0.25 \leq y \leq 0.35)$. 


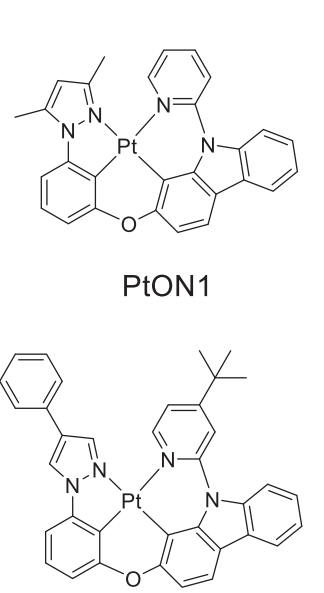

PtON6-tBu (3-4)

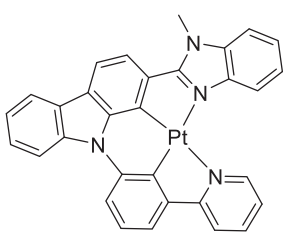

PtN8ppy (3-9)

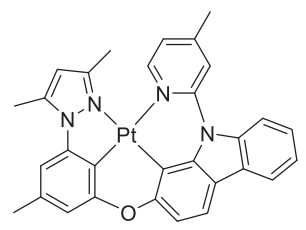

Pt(ppzOczpy-4m) (3-13)

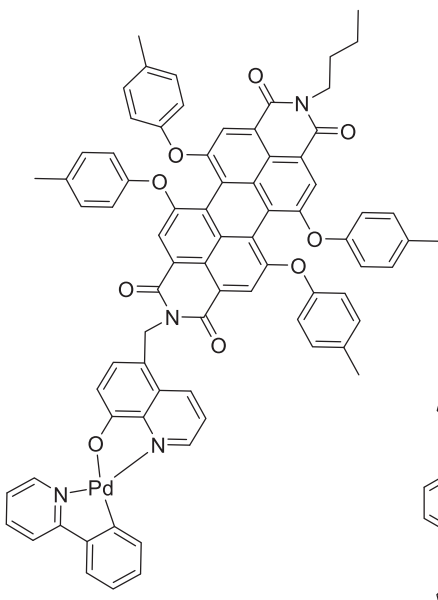

(Phpy)Pd(Pery-q)

(3-17)

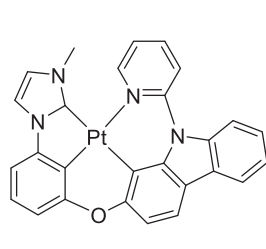

PtON7
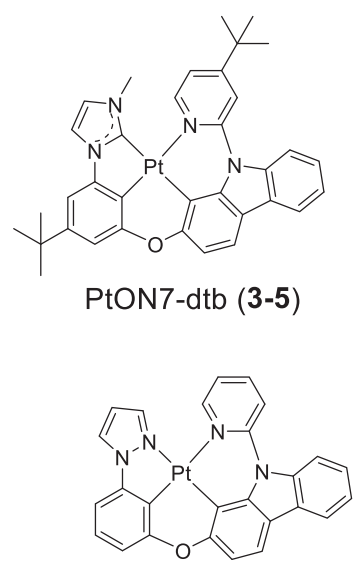

Pt(ppzOczpy) (3-10)

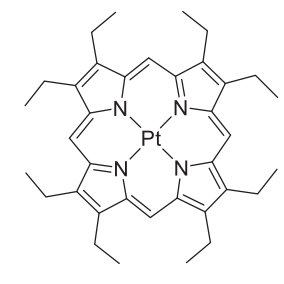

PtOEP (3-14)
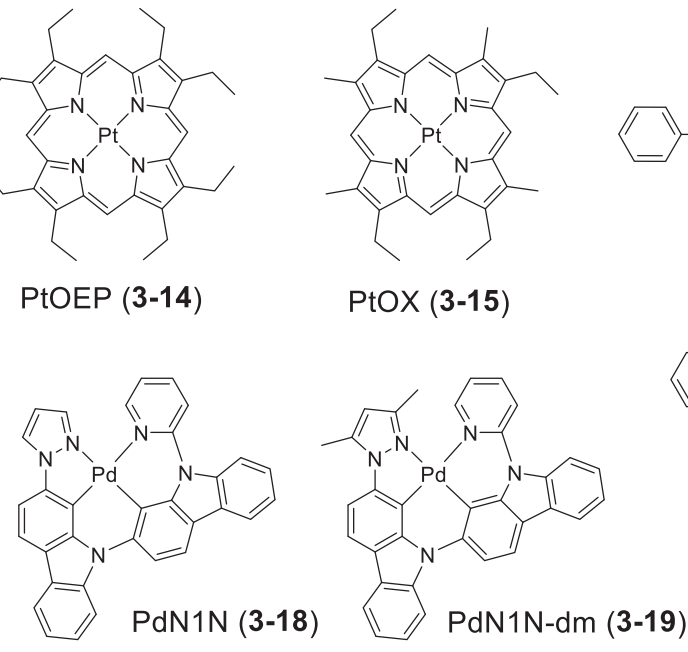

PtOX (3-15)

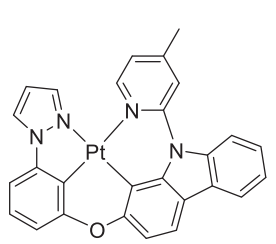

Pt(ppzOczpy-m) (3-11)
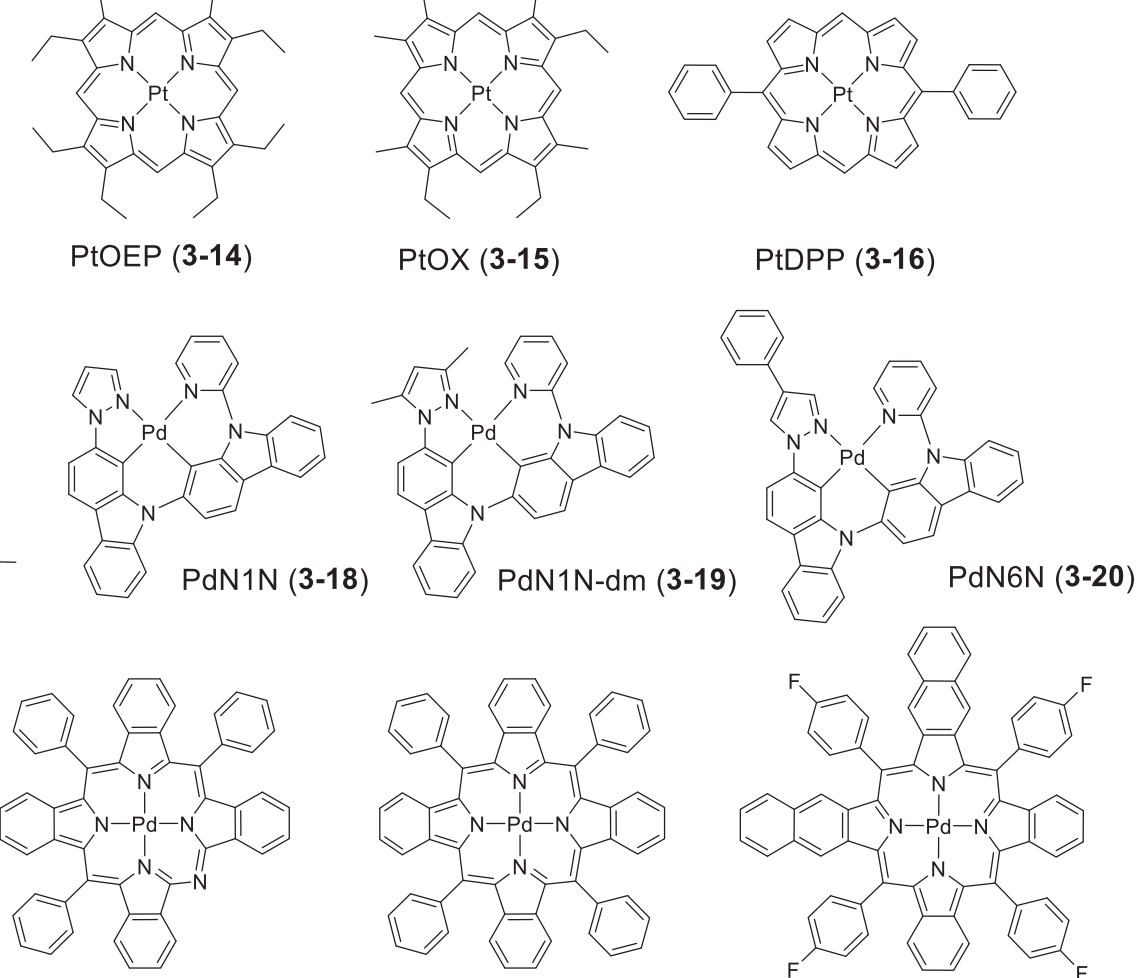

PdNTBP (3-21)

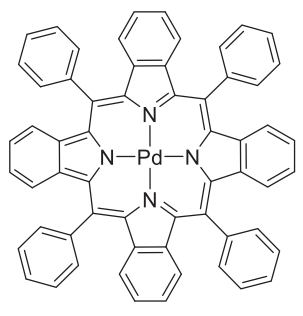

PdTPTBP (3-22)
PtDPP (3-16)

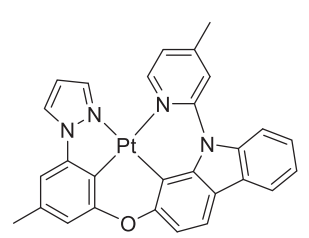

Pt(ppzOczpy-2m) (3-12)

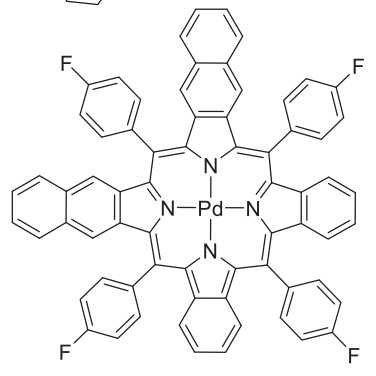

Pd2NF (3-23)

Fig. 8 Chemical structures of Pt- and Pd-based cyclometalated complexes. 


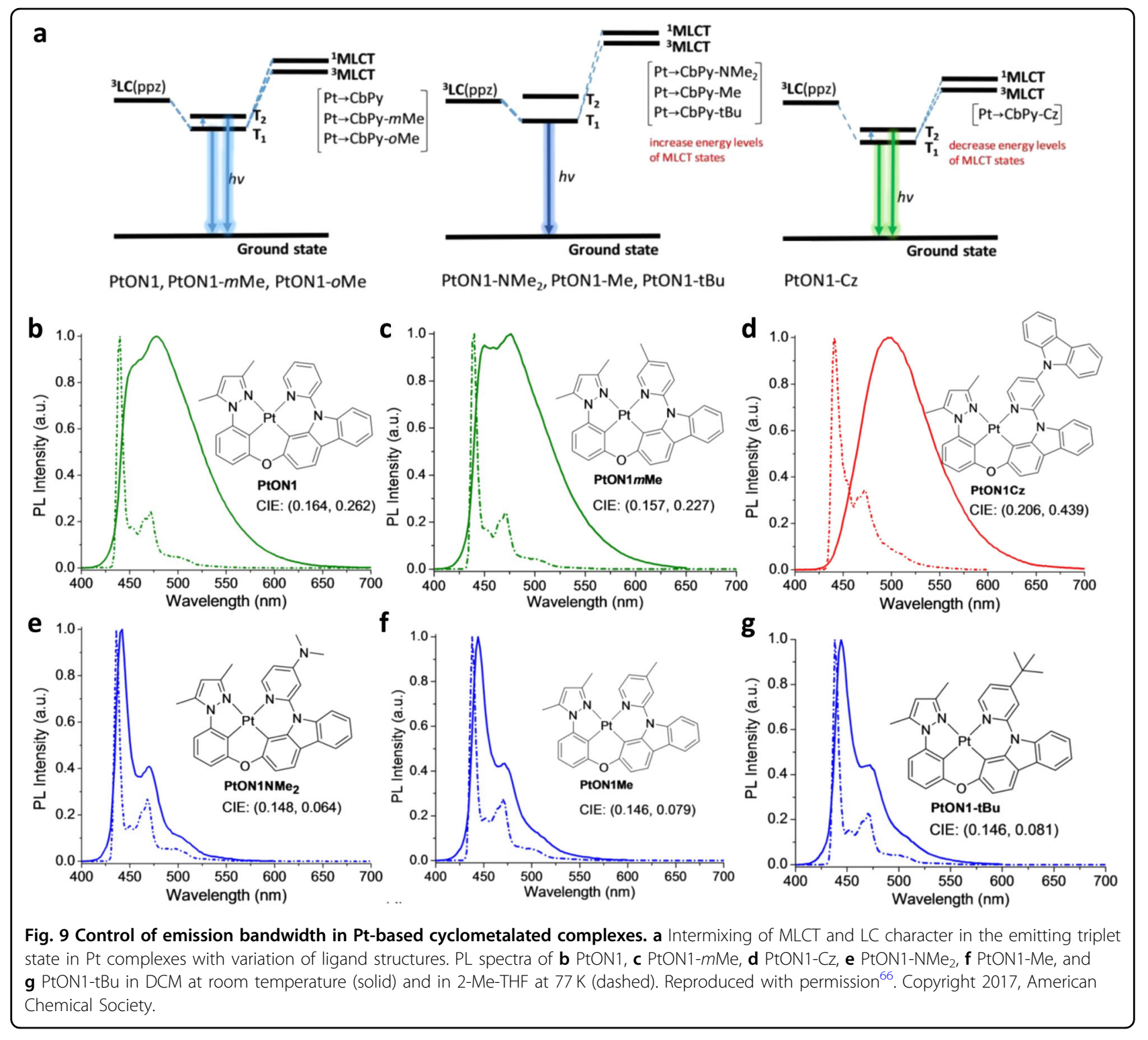

Despite the fact that $\mathrm{Pt}$ and $\mathrm{Pd}$ are both group 10 transition metals (and would therefore be expected to have similar properties), there are fewer studies on phosphorescent emitters based on Pd than on their Pt counterparts because their lowest-lying excited states are usually depleted by the thermally accessible MC states that deactivate radiative decay at room temperature ${ }^{72}$. Zhu et al. designed a Pd complex ((Phpy)Pd(Pery-q), 3-17) with a 2-phenylpyridine and hydroxyquinolinesubstituted perylene bisimide as an asymmetric ligand ${ }^{73}$. Introducing perylene bisimide can prevent the localization of electrons in the thermally accessible MC state by causing the ligand localized (intraligand) excited state to be below the ${ }^{3}$ MLCT state. Consequently, once the singlet excited state of perylene bismide is populated, it acts as a phosphorescent sensitizer and undergoes ISC to the triplet state of the hydroxyquinoline ligand. Finally, narrow phosphorescence spectra were measured at $635 \mathrm{~nm}$ (FWHM = $27 \mathrm{~nm})$ in DCM and $625 \mathrm{~nm}($ FWHM $=18 \mathrm{~nm})$ in $\operatorname{poly}(9-$ vinylcarbazole) film at room temperature.

New Pd-containing phosphorescent blue emitters, PdN1N (3-18), PdN1N-dm (3-19), and PdN6N (3-20), were prepared by introducing pyridylcarbazole and pyrazolylcarbazole-based tetradentate ligands to achieve narrow emission with $S<0.5$ (FWHM $18 \sim 22 \mathrm{~nm}$ ) at $\lambda_{\mathrm{PL}}=470-476 \mathrm{~nm}$ in $\mathrm{DCM}^{74}$. Borisov et al. reported the Pd-phorphyrin type complexes PdNTBP (3-21) and PdTPTBP (3-22), which showed strong room temperature red phosphorescence in deoxygenated toluene with a narrow-bandwidth (FWHM $=32 \mathrm{~nm}$ and $47 \mathrm{~nm}$, respectively) ${ }^{75}$. Additionally, Niedermair et al. synthesized a series of Pd porphyrin complexes by fusing an additional benzene 
ring onto at least one of the benzo groups in tetrabenzoporpyrin ${ }^{76}$. Extension of the aromaticity in the porphyrin framework provided emission maxima in $\mathrm{Pd}$ complexes in the near infrared region $(849-882 \mathrm{~nm})$. In particular, cis-palladium(II)-meso-tetra-(4-fluorophenyl) dibenzodinaphthoporphyrin (Pd2NF, 3-23) exhibited PL at $868 \mathrm{~nm}$ with a small FWHM of $41 \mathrm{~nm}$ in toluene. Similar to those of Pt porphyrins, the optical properties of Pd porphyrins are determined by the degree of similarity between the ground and excited states of the porphyrin ligands.

In addition, industries have also tried to develop narrowband emitters; for example, deep red-emitting phosphorescent OLEDs with suppressed first vibronic transition in their EL spectra (molecular structure was not disclosed, $\lambda_{\mathrm{E}}=640 \mathrm{~nm}$ with a FWHM of $43 \mathrm{~nm}$ ) were reported by Universal Display Co. and Beijing Summer Sprout Technology Co., Ltd. at the Society for Information Display (SID) conference in 2019 and 2020, respectively ${ }^{77,78}$.

\section{Eu- and Tb-based phosphorescent cyclometalated complexes}

Owing to their sharp emission, trivalent lanthanide (Ln) metal complexes, including those with $\mathrm{Eu}^{3+}$ and $\mathrm{Tb}^{3+}$ ions, have attracted attention as red- and green-emissive phosphorescent materials (Fig. 10) ${ }^{79}$. The luminance of $\mathrm{Eu}$ and $\mathrm{Tb}$ complexes is mainly caused by $f \rightarrow f$ transitions in the metal and shows an extremely sharp emission (FWHM $<10 \mathrm{~nm}$ ). Because the direct population of the excited emitting state in $\mathrm{Ln}^{3+}$ is prohibited, the organic ligands in the Ln complex were first excited, and then the $\mathrm{T}_{1}$ state was formed. Subsequently, Dexter-type electron exchange between the $T_{1}$ state of the ligand and the $\mathrm{Ln}$ ion induces the indirect population of the $4 \mathrm{f}$ level of $\mathrm{Ln}^{3+}$ complexes, yielding sensitized luminescence ${ }^{80-82}$. In Ln metal complexes, ligands determine the sensitization phenomenon; therefore, the resulting PL intensity of the Ln ion is different depending on the structure of the ligand ${ }^{83}$. In Eu complexes, $\beta$-diketonate-type structures have been widely used as ligands to sensitize $\mathrm{Eu}^{3+}$ ions, where control of the precise energy of the ligand $T_{1}$ energy level is important to ensure that it has a higher energy than the emissive level of $\mathrm{Eu}^{3+}\left({ }^{5} \mathrm{D}_{0}\right)$ and to optimize the sensitization. Martín-Gil et al. synthesized $\mathrm{Eu}(\mathrm{cbtfa})_{3}$ (bath) (4-1), which has a halogenated $\beta$-diketonate-based ligand and a rigid bathophenanthroline ancillary ligand (bath) ${ }^{84}$. Upon excitation at $365 \mathrm{~nm}$, the $\mathrm{Eu}(\mathrm{cbtfa})_{3}$ (bath) powder, solution (in chloroform), and thin film showed almost identical PL spectra with a sharp red emission at $613 \mathrm{~nm}$ with a $5 \mathrm{~nm}$ FWHM. As shown in Fig. 11a, a strong electric dipole transition $\left({ }^{5} \mathrm{D}_{0}{ }^{7} \mathrm{~F}_{2}\right)$ causes intense emission at $613 \mathrm{~nm}$ due to the highly polarizable ligand field around the $\mathrm{Eu}^{3+}$ ion and has five satellite peaks corresponding to intraconfigurational $f \rightarrow f$ transitions. The EL spectra of the EL (ITO/PEDOT:PSS/
$\left.\mathrm{Eu}(\mathrm{cbtfa})_{3}(\mathrm{bath}) / \mathrm{Ca} / \mathrm{Al}\right)$ device were similar to the PL spectra with a dominant emission at $613 \mathrm{~nm}$. Because only one crystal field line is possible for a complex with $D_{3 h}$ symmetry ${ }^{79}$, emission from most Eu complexes occurs from a narrow ${ }^{5} \mathrm{D}_{0} \rightarrow{ }^{7} \mathrm{~F}_{2}$ transition, regardless of the external environment or ligand structures. Sivakumar et al. synthesized $\mathrm{Eu}(\mathrm{TTA})_{3}$ Phen-Fl-TPA (4-2), in which the tta ligand acts as an optical antenna with efficient energy transfer to $\mathrm{Eu}^{3+}$ ions, exhibiting a sharp emission at $612 \mathrm{~nm}$ in both solution and film samples ${ }^{85}$. Borisov et al. reported a series of Eu complexes, $\mathrm{Eu}(\mathrm{HPhN})_{3}$ phen (4-3), $\mathrm{Eu}(\mathrm{HPhN})_{3} \mathrm{dpp}(4-4)$, and $\mathrm{Eu}(\mathrm{HPhN})_{3} \mathrm{DDXPO}$ (4-5), containing 9-hydroxy-1 $H$-phenalen-1-one (HPhN) as a sensitizing ligand and different ancillary ligands ${ }^{86} . \mathrm{S}_{1}$ and $T_{1}$ excited states of the ancillary ligands with a higher energy than those of $\mathrm{HPhN}$ improved the sensitization process, and similar PL spectra at $\sim 611 \mathrm{~nm}$ were observed for all structures, showing $20 \%$ PLQY in polystyrene films. Similar results were reported by Vaidyanathan et al., who showed that all Eu- $\beta$-diketonate complexes had similar PL data with a sharp emission at $612 \mathrm{~nm}$ both in film and in chloroform solution, regardless of the ancillary ligand (4-6 4-10), indicating efficient energy transfer from the $T_{1}$ state of the $\beta$-diketonate ligand to the excited state of $\mathrm{Eu}^{3+}$ (Fig. 11b) ${ }^{87}$.

Similarly, various $\mathrm{Tb}$ complexes with different ligands also exhibit similar PL spectra arising from the ${ }^{5} \mathrm{D}_{4} \rightarrow{ }^{7} \mathrm{~F}_{6}$ transition (Fig. $11 \mathrm{c})^{88}$. Huang et al. reported a Tb complex, $\mathrm{Tb}(\mathrm{PMIP})_{3} \mathrm{DPPOC}(\mathbf{4}-\mathbf{1 1})$, whose optical and electrical properties were comparable to those of $\mathrm{Tb}$ $(\mathrm{PMIP})_{3}\left(\mathrm{H}_{2} \mathrm{O}\right)_{2}$ (4-12) and $\mathrm{Tb}(\mathrm{PMIP})_{3}(\mathrm{TPPO})_{2}$ (4-13) (Fig. 11d ${ }^{89}$. Fig. 11d also shows that similar emission spectra were observed for all $\mathrm{Tb}$ complexes with sharp $\mathrm{Tb}^{3+}$ emission at $548 \mathrm{~nm}\left({ }^{5} \mathrm{D}_{4} \rightarrow{ }^{7} \mathrm{~F}_{5}\right)$ with multiple satellite peaks. Since the energy level of the triplet state of DPPOC is closer to the ${ }^{5} \mathrm{D}_{4}$ level of $\mathrm{Tb}, \mathrm{Tb}(\mathrm{PMIP})_{3} \mathrm{DP}-$ POC showed a higher PLQY (16.7\%) than the other Tb complexes. When the Tb(PMIP) $)_{3}$ DPPOC-based EL device was fabricated with an architecture of ITO/NPB/Tb $(\mathrm{PMIP})_{3} /$ co-deposited $\mathrm{Tb}(\mathrm{PMIP})_{3} \mathrm{DPPOC} / \mathrm{BCP} / \mathrm{Alq}_{3} /$ $\mathrm{Mg}_{0.9} \mathrm{Ag}_{0.1} / \mathrm{Ag}$, strong green emission from the $\mathrm{Tb}$ ions was observed with a maximum brightness of $2256 \mathrm{~cd} \mathrm{~m}^{-2}$ and power efficiency of $16.1 \mathrm{~lm} \mathrm{~W}^{-1}$ (at $5 \mathrm{~V}$ ). Raithby et al. synthesized a new $\mathrm{Tb}$ complex, $\mathrm{Tb}(\mathrm{tfac})_{3} \mathrm{DPEPO}(\mathbf{4}-\mathbf{1 4})$, with neutral bidentate ligands, trifluoroacetylacetone, and $\mathrm{DPEPO}^{90}$. Both the PL and EL spectra of $\mathrm{Tb}(\mathrm{tfac})_{3} \mathrm{DPEPO}$ were very similar, with emission maxima at $\sim 545 \mathrm{~nm}$.

\section{TADF-based light-emitting materials}

TADF, which was pioneered by Parker and Hatchard, is a phenomenon whereby triplet excitons are converted to singlet excitons by thermal activation and consequently undergo fluorescence ${ }^{91}$. Theoretically, $100 \%$ internal quantum efficiency (IQE) is possible via an efficient 


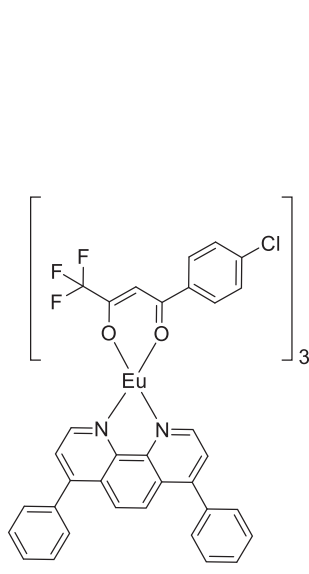

$\mathrm{Eu}(\mathrm{cbtfa})_{3}$ (bath)

(4-1)

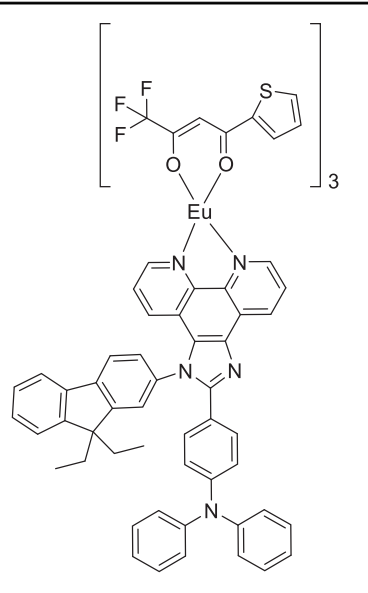

$\mathrm{Eu}(\mathrm{TTA})_{3}$ Phen-FI-TPA

(4-2)

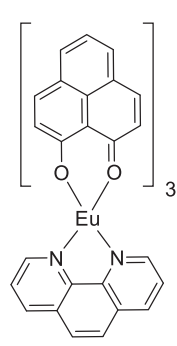

$\mathrm{Eu}(\mathrm{HPhN})_{3}$ phen

(4-3)

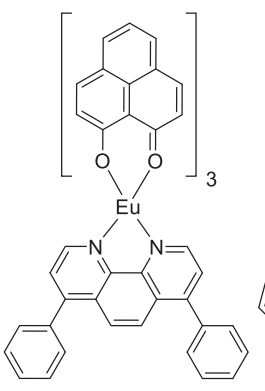

$\mathrm{Eu}(\mathrm{HPhN})_{3} \mathrm{dpp}$

(4-4)

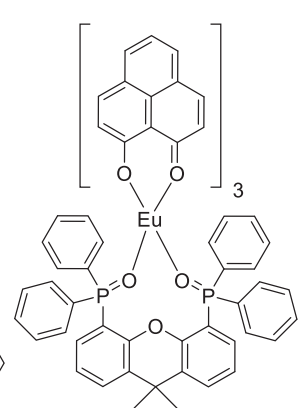

$\mathrm{Eu}(\mathrm{HPhN})_{3} \mathrm{DDXPO}$

(4-5)

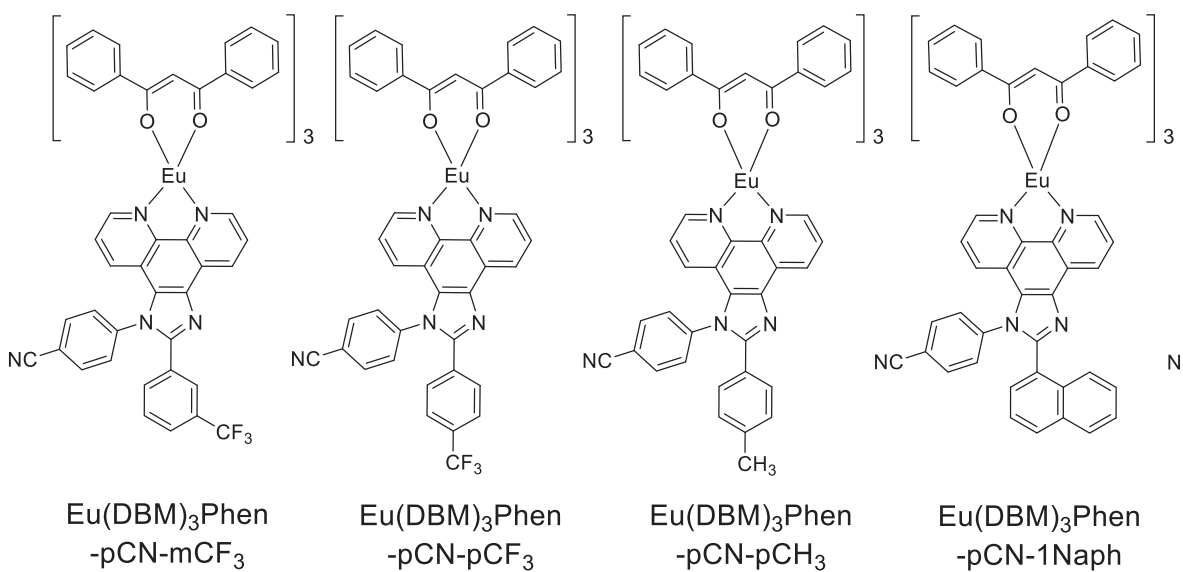

(4-6)

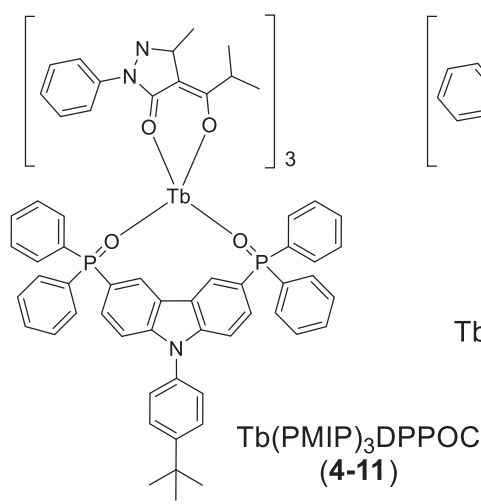

(4-8)

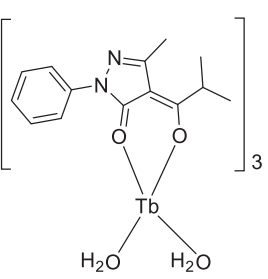

$\mathrm{Tb}(\mathrm{PMIP})_{3}\left(\mathrm{H}_{2} \mathrm{O}\right)_{2}$

(4-12)

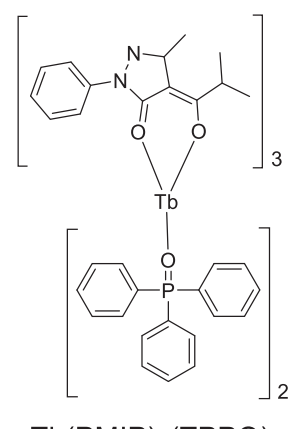

$\mathrm{Tb}(\mathrm{PMIP})_{3}(\mathrm{TPPO})_{2}$

(4-13)
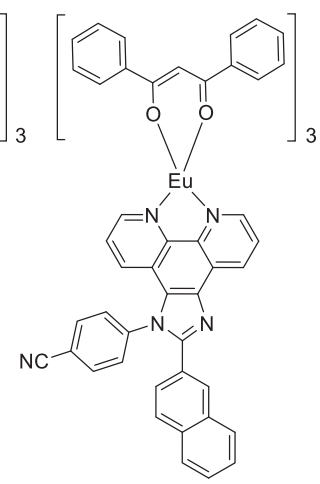

\section{$\mathrm{Eu}(\mathrm{DBM})_{3}$ Phen $-p C N-2 N a p h$}

(4-10)

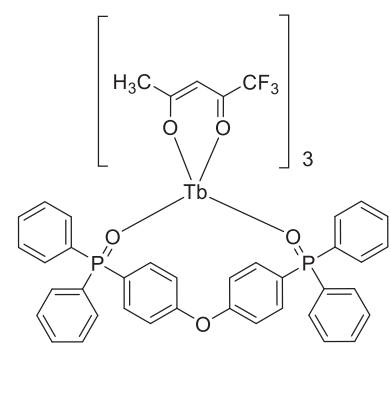

$\mathrm{Tb}(\mathrm{tfac})_{3} \mathrm{DPEPO}$

(4-14)

Fig. 10 Chemical structures of Eu and Tb complexes.

upconversion process called reverse intersystem crossing (RISC). Adachi's group successfully developed a new generation of TADF-based OLEDs ${ }^{92}$. The design of TADF emitters involves spatial separation of the electron-rich (donor) and electron-deficient (acceptor) moieties to separate the HOMO and LUMO distributions and create a small energy gap $\left(\Delta E_{\mathrm{ST}}\right)$ between the $\mathrm{S}_{1}$ and $\mathrm{T}_{1}$ states (Fig. 12a) ${ }^{93}$. This design strategy has resulted in a significant improvement in EL efficiency, but it also enhances the structural relaxation in the excited states via ICT, resulting in broad CT emission (FWHM of $70-100 \mathrm{~nm}$ ) with a large Stokes shift in TADF OLED devices (Fig. 12b) ${ }^{94}$. 
a

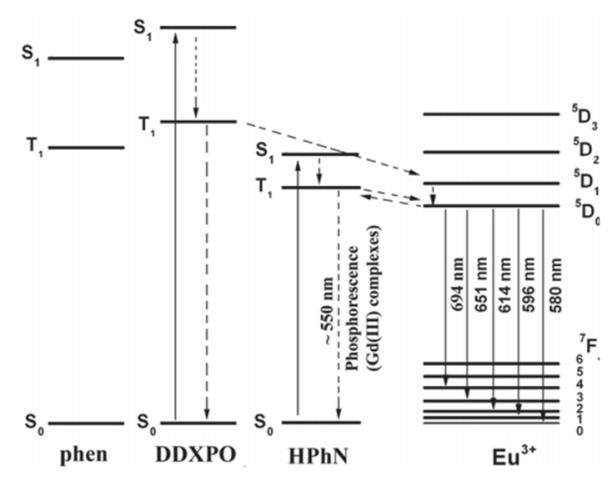

C Organic ligand states

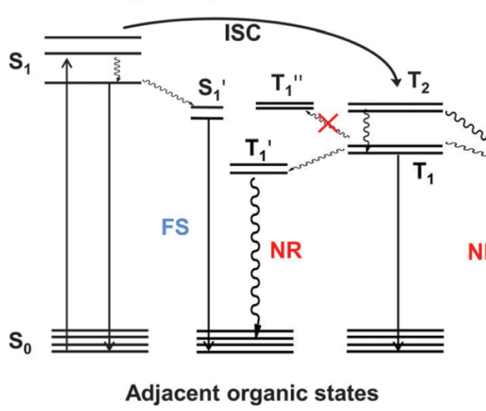

b

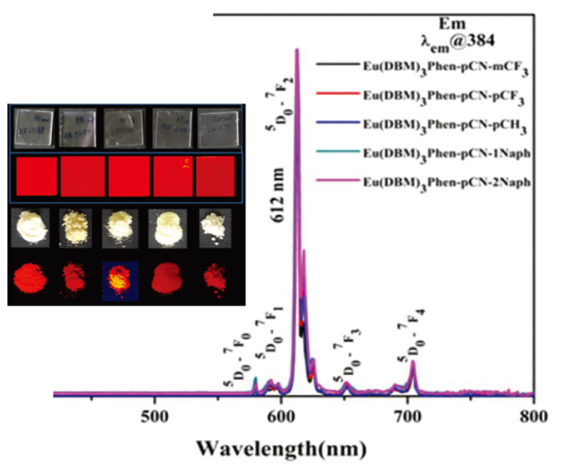

d

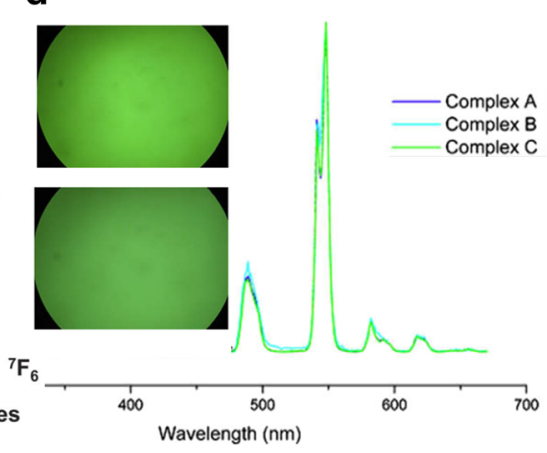

Fig. 11 Light-emitting characteristics of Eu-and Tb-containing cyclometalated complexes. a Energy level diagram of Eu(III) metal and respective ligands (phen, DDXPO, and $\mathrm{HPhN}$ ) in Eu complexes. Reproduced with permission ${ }^{86}$. Copyright 2014, Wiley-VCH. b PL spectra of Eu(DBM) $3^{-}$ based complexes with different ancillary ligands in chloroform (inset: thin film and powdered Eu complexes under daylight and UV irradiation at $365 \mathrm{~nm}$ ). Reproduced with permission ${ }^{87}$. Copyright 2020, The Royal Society of Chemistry. c Energy transfer in Tb complexes. Reproduced with permission $^{88}$. Copyright 2016, The Royal Society of Chemistry. $\mathbf{d}$ PL spectra of Tb complexes in ethanol (complex $A=T b(P M I P)_{3}(D P P O C) ; B=T b$ $(\mathrm{PMIP})_{3}\left(\mathrm{H}_{2} \mathrm{O}\right)_{2} ; \mathrm{C}=\mathrm{Tb}(\mathrm{PMIP})_{3}(\mathrm{TPPO})_{2}$, inset: fluorescence microscopy image of complexes A-C films). Reproduced with permission ${ }^{89}$. Copyright 2009, Elsevier.

To achieve both high-performance and narrowband emission in TADF OLEDs, Hatakeyama et al. designed new TADF materials in which a rigid molecular framework with regular arrangements of boron and nitrogen atoms shows an MR effect; the HOMO is localized on the nitrogen atoms and at the meta-position with respect to the boron atom, whereas the LUMO is localized on the boron atom and at the ortho- and para-positions (Fig. 12c) ${ }^{94}$. Thus, the boron and nitrogen atoms in the MR-TADF structures have the opposite resonance effect, and the HOMO and LUMO are separated onto different atoms without the need for electron-rich or electrondeficient substituents. The MR-TADF compounds shown in Fig. 13 undergo a limited amount of reorganization in the excited state and maintain their oscillating strength due to effective overlap of the electron and hole wavefunctions, ensuring narrow PL and EL spectra with high luminescence efficiency. In addition, the MR effect minimizes the bonding/antibonding characteristics of the HOMO and LUMO, and the resulting nonbonding MOs minimize the vibronic coupling and vibrational relaxation in the material, resulting in emission peaks with an extremely small $\mathrm{FWHM}^{95}$. In this chapter, various MRTADF- and conventional D-A-type molecules are summarized, and strategies to achieve narrow emission are discussed (Table 3).

\section{Multiple resonance (MR) TADF emitters}

In 2015, Hatakeyama et al. first reported a series of TADF dopants, 5,9-dioxa-13b-boranaphtho[3,2,1-de] anthracene (5-1), 2,12-phenyl-5,9-dioxa-13b-boranaphtho [3,2,1-de] anthracene (5-2), 3,11-diphenyl-5,9-dioxa-13bboranaphtho[3,2,1-de] anthracene (5-3), and 6,8-diphenyl5,9-dioxa-13b-boranaphtho[3,2,1-de] anthracene $\quad(5-4)^{96}$. In compound 5-1, separation of the localized HOMO and LUMO suppresses the exchange interaction between MOs, enhancing the resonance effects of boron and oxygen atoms in the excited state. As a result, compounds 51 3 showed strong and narrow PL profiles at 398 410 nm with FWHM $<33 \mathrm{~nm}$ in DCM, while 5-4 showed a slightly broader emission (FWHM $=49 \mathrm{~nm}$ ) attributed to ICT. Based on the observed structure-property relationships, 

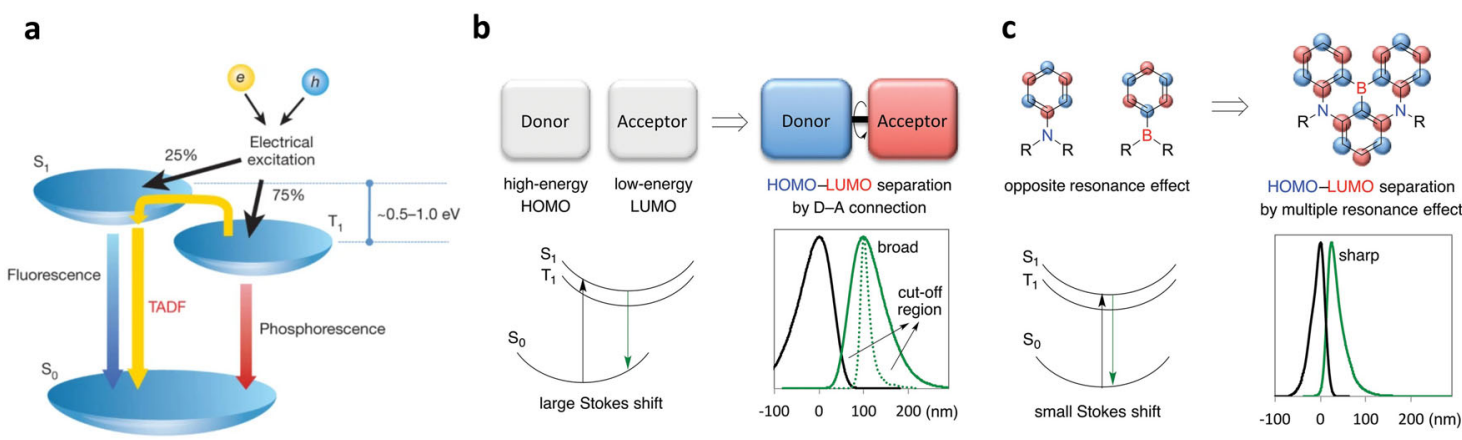

Fig. 12 Light-emitting characteristics of TADF emitters. a Schematic diagram of TADF. Reproduced with permission ${ }^{93}$. Copyright 2012, Springer Nature. b Molecular design strategy to control the HOMO/LUMO distribution. Absorption (black) and emission (green) spectra of TADF materials. Typical TADF emitters show a broad emission peak due to their CT character. c Molecular design strategy to control HOMO and LUMO distribution in boron-containing MR-TADF materials. Narrow absorption (black) and emission (green) spectra of MR-TADF materials. b, c Reproduced with permission ${ }^{94}$. Copyright 2016, Wiley-VCH.

the Hatakeyama group further developed various narrowemissive blue TADF emitters with rigid boron-containing polycyclic aromatic hydrocarbons (PAHs). In particular, 5,9-diphenyl-5,9-diaza-13b-boranaphtho[3,2,1-de]anthracene (DABNA1, 5-5) and 9-([1,1'-biphenyl]-3-yl)- $N$, N,5,11-tetraphenyl-5,9-dihydro-5,9-diaza-13b-boranaphtho [3,2,1-de]anthracen-3-amine (DABNA2, 5-6) are considered to be representative narrow-emissive TADF materials ${ }^{94}$. Unlike those of conventional TADF emitters, the HOMO and LUMO of DABNA1 and DABNA2 are welldistributed over the whole boron-based molecular framework by the MR effect. DABNA1 and DABNA2 both have a small $\Delta E_{\mathrm{ST}}(0.20 \mathrm{eV})$ and bright blue emission at $\lambda_{\mathrm{PL}}=$ $460-470 \mathrm{~nm}$ with a small FWHM $(28-36 \mathrm{~nm})$ in an mCBP film and even in a polar solvent (ethanol). Additionally, the small Stokes shifts of DABNA1 and DABNA2 (25 and $26 \mathrm{~nm}$, respectively) are attributed to the rigid, conjugated core. A DABNA-based OLED with a device structure of ITO/NPD/TCTA $/ \mathrm{mCP} / \mathrm{mCBP}: 1 \mathrm{wt} \%$ DABNA1 or 2/TSPO1/LiF/Al showed a narrower EL spectrum at $\lambda_{\mathrm{EL}}=459 \mathrm{~nm}$ (DABNA1) and $\lambda_{\mathrm{EL}}=467 \mathrm{~nm}$ (DABNA2, $\mathrm{FWHM}=28 \mathrm{~nm}$ for both devices) compared to their PL spectra, with well-matched blue CIE coordinates (BT 2020) (Fig. 14a, b). However, the device doped with $1 \mathrm{wt} \%$ DABNA faced severe efficiency roll-off owing to the charge imbalance and bimolecular quenching process. Although the efficiency roll-off improved at a higher emitter doping concentration ( $5 \mathrm{wt} \%)$, the EL spectrum of DABNA2 was redshifted $(467 \mathrm{~nm})$. The study highlighted the importance of substituents on rigid $\mathrm{B} / \mathrm{N}$ cores to localize the $\mathrm{HOMO}$ and LUMO at the core and thereby maintain their emission color purities.

Following Oi et al.'s report of a triangulene-shaped planaroborane compound, 4,8,12-trioxa-12c-boradibenzo[cd, $\mathrm{mn}]$ pyrene (5-7), which showed a narrow PL emission at
$\lambda_{\mathrm{PL}}=386 \mathrm{~nm}$ with an FWHM of $47 \mathrm{~nm}$ in toluene ${ }^{97}$, Hatakeyama modified the triangulene core by replacing the bridging oxygen atoms with nitrogen. This modification resulted in a small PL bandwidth (FWHM $=26 \mathrm{~nm}$ ) at $\lambda_{\mathrm{PL}}=399 \mathrm{~nm}$ for 5-8 in the PMMA film together with a small $\Delta \mathrm{E}_{\mathrm{ST}}$ of $0.21 \mathrm{eV}^{98}$. In the presence of $\mathrm{N}$ atoms, $\mathrm{X}$-ray crystallography showed that the $\mathrm{B}-\mathrm{C}$ bond of $\mathbf{5 - 8}$ is shorter $(1.478-1.480 \AA)$ than that of triphenyl boron (1.571-1.589 $\AA$ ), emphasizing that the planarity is strongly enhanced by the bridging $\mathrm{N}$ atoms. This planar polycyclic $\mathrm{B} /$ $\mathrm{N}$ skeleton was further developed to form $\mathrm{B} / \mathrm{N}$-doped nanographene structures, B2 (5-9), B3 (5-10), and B4 (5-11), extending the $\pi$ conjugation of the PAHs with an increased number of boron atoms ${ }^{99}$. B2, B3, and B4 all showed narrow blue emissions with FWHM $=32$, 34, and $38 \mathrm{~nm}$ in PMMA films, respectively. In particular, highpurity blue emissions with CIE coordinates of $(0.13,0.11)$, $\lambda_{\mathrm{E}}=460 \mathrm{~nm}$, and a maximum EQE of $18.3 \%$ at $1 \mathrm{~cd} \mathrm{~m}^{-2}$ were observed from B2 OLEDs. Concurrently, DABNA structures were modified by switching the positions of the boron and nitrogen atoms, yielding 5,9-bis(2,6-dimethylphenyl)-3,7,11-trimethyl-5,9-dihydro-13b-aza-5,9-diboranaphtho[3,2,1-de]anthracene (ADBNA-Me-Mes, 5-12) and 3,7,11-trimethyl-5,9-bis(2,4,6-triisopropylphenyl)-5,9dihydro-13b-aza-5,9-diboranaphtho[3,2,1-de]anthracene (ADBNA-Me-Tip, 5-13) ${ }^{100}$. When doped into the DOBNA-OAr film, both ADBNA derivatives showed narrow emission (FWHM $\sim 34 \mathrm{~nm}$ ) in the sky blue region $\left(\lambda_{\mathrm{PL}}=482\right.$ and $479 \mathrm{~nm}$ for ADBNA-Me-Mes and ADBNAMe-Tip, respectively). In particular, fabricated ADBNA-MeTip devices with a structure of ITO/HATCN/NPB/TCTA/ mCP/DOBNA-OAr:1 wt\% ADBNA-Me-Tip/TSPO1/LiF/Al showed narrow EL spectra with $F W H M=33 \mathrm{~nm}$ and maximum $\mathrm{EQE}=21.4 \%$ with $\Delta \mathrm{E}_{\mathrm{ST}}=0.20 \mathrm{eV}$ and efficient RISC $\left(k_{\text {RISC }}=9.0 \times 10^{3} \mathrm{~s}^{-1}\right)$ (Fig. 14c). Although efficiency 
Table 3 Summary of optical and device properties of narrow-bandwidth TADF emitters.

\begin{tabular}{|c|c|c|c|c|c|c|c|c|c|}
\hline No. & Emitter & $\lambda_{\mathrm{PL}}(\mathrm{nm})$ & $\mathrm{FWHM}_{\mathrm{PL}}(\mathrm{nm})$ & PLQY (\%) & $\lambda_{\mathrm{EL}}(\mathrm{nm})$ & $\mathrm{FWHM}_{\mathrm{EL}}(\mathrm{nm})$ & EQE (\%) & $\mathrm{ClE}_{x, y}$ & Refs. \\
\hline \multicolumn{10}{|c|}{ Multiple resonance (MR) TADF emitters } \\
\hline $5-1$ & $2 a$ & 398 (in DCM) & $32^{*}$ (in DCM) & 72 (in PMMA film) & NA & NA & NA & NA & \multirow[t]{5}{*}{96} \\
\hline $5-2$ & $2 b$ & 410 (in DCM) & $27^{*}$ (in DCM) & 65 (in PMMA film) & NA & NA & NA & NA & \\
\hline $5-3$ & $2 c$ & 410 (in DCM) & $33^{*}$ (in DCM) & 60 (in PMMA film) & NA & NA & NA & NA & \\
\hline $5-4$ & $2 d$ & 436 (in DCM) & $49^{*}$ (in DCM) & 57 (in PMMA film) & NA & NA & NA & NA & \\
\hline $5-5$ & DABNA $1^{a}$ & $\begin{array}{l}462 \text { (in DCM) } \\
458 \text { (in EtOH) } \\
460 \text { (in } \mathrm{mCBP} \text { film) }\end{array}$ & $\begin{array}{l}33 \text { (in } \mathrm{DCM} \text { ) } \\
36 \text { (in } \mathrm{EtOH} \text { ) } \\
30 \text { (in } \mathrm{mCBP} \text { film) }\end{array}$ & $\begin{array}{l}89 \text { (in } \mathrm{DCM} \text { ) } \\
84 \text { (in } \mathrm{EtOH} \text { ) } \\
88 \text { (in } \mathrm{mCBP} \text { film) }\end{array}$ & 459 & 28 & 13.5 & $(0.13,0.09)$ & \\
\hline $5-6$ & DABNA2 $2^{\mathrm{a}}$ & $\begin{array}{l}470 \text { (in DCM) } \\
463 \text { (in EtOH) } \\
469 \text { (in } \mathrm{mCBP} \text { film) }\end{array}$ & $\begin{array}{l}34 \text { (in } \mathrm{DCM} \text { ) } \\
34 \text { (in } \mathrm{EtOH} \text { ) } \\
28 \text { (in } \mathrm{mCBP} \text { film) }\end{array}$ & $\begin{array}{l}85 \text { (in } \mathrm{DCM} \text { ) } \\
90 \text { (in } \mathrm{EtOH} \text { ) } \\
90 \text { (in } \mathrm{mCBP} \text { film) }\end{array}$ & 467 & 28 & 20.2 & $(0.12,0.13)$ & \\
\hline $5-7$ & 1 & 386 (in Tol) & $47^{*}$ (in Tol) & 63.2 (in Tol) & NA & NA & NA & NA & 97 \\
\hline $5-8$ & $2 \mathrm{a}$ & 399 (in PMMA film) & 26 (in PMMA film) & 54 (in PMMA film) & NA & NA & NA & NA & 98 \\
\hline $5-9$ & $\mathrm{~B} 2^{\mathrm{a}}$ & 45 (in PMMA film) & 32 (in PMMA film) & 53 (in PMMA film) & 460 & 37 & 18.3 & $(0.13,0.11)$ & 99 \\
\hline $5-10$ & B3 & 441 (in PMMA film) & 34 (in PMMA film) & 33 (in PMMA film) & NA & NA & NA & NA & \\
\hline $5-11$ & B4 & 450 (in PMMA film) & 38 (in PMMA film) & 57 (in PMMA film) & NA & NA & NA & NA & \\
\hline $5-12$ & ADBNA-Me-Mes ${ }^{b}$ & $\begin{array}{l}484 \text { (in DCM) } \\
482 \text { (in DOBNA- } \\
\text { OAr film) }\end{array}$ & $\begin{array}{l}38 \text { (in DCM) } \\
3 \text { (in DOBNA- } \\
\text { OAr film) }\end{array}$ & $\begin{array}{l}89 \text { (in DOBNA- } \\
\text { OAr film) }\end{array}$ & 481 & 32 & 16.2 & $(0.10,0.27)$ & 100 \\
\hline $5-13$ & ADBNA-Me-Tip ${ }^{b}$ & $\begin{array}{l}482 \text { (in DCM) } \\
479 \text { (in DOBNA- } \\
\text { OAr film) }\end{array}$ & $\begin{array}{l}36 \text { (in DCM) } \\
34 \text { (in DOBNA- } \\
\text { OAr film) }\end{array}$ & $\begin{array}{l}88 \text { (in DOBNA- } \\
\text { OAr film) }\end{array}$ & 480 & 33 & 21.4 & $(0.11,0.29)$ & \\
\hline $5-14$ & $v-D A B N A^{c}$ & $\begin{array}{l}468 \text { (in Tol) } \\
467 \text { (in DOBNA- } \\
\text { OAr film) }\end{array}$ & $\begin{array}{l}14 \text { (in Tol) } \\
18 \text { (in DOBNA- } \\
\text { OAr film) }\end{array}$ & $\begin{array}{l}74 \text { (in Tol) } \\
90 \text { (in DOBNA- } \\
\text { OAr film) }\end{array}$ & 469 & 18 & 34.4 & $(0.12,0.11)$ & 95 \\
\hline $5-15$ & $t-D A B N A^{d}$ & NA & NA & $\begin{array}{l}88 \text { (in DPEPO:DMAC- } \\
\text { DPS film) }\end{array}$ & $450^{*}$ & 31 & 31.4 & $(0.13,0.15)$ & 101 \\
\hline $5-16$ & TBN-TPA & 470 (in Tol) & 26 (in Tol) & 97.5 (in Tol) & 474 & 27 & 32.1 & $(0.12,0.19)$ & 102 \\
\hline $5-17$ & $\begin{array}{l}\text { DtBuCzB (or } \\
\text { BBCz-SB) }\end{array}$ & 481 (in Tol) & 22 (in Tol) & 91 (in Tol) & 488 & 29 & 21.6 & $(0.10,0.42)$ & 104 \\
\hline $5-18$ & $\mathrm{DtBuPhCzB}$ & 496 (in Tol) & 21 (in Tol) & 97 (in Tol) & $\begin{array}{l}504^{f}, 504^{g} \\
508^{h}\end{array}$ & $34^{f}, 33^{g}, 33^{h}$ & $\begin{array}{l}23.4^{f}, 23.5^{g} \\
25.5^{h}\end{array}$ & $\begin{array}{l}(0.15,0.61)^{f} \\
(0.19,0.61)^{g} \\
(0.20,0.65)^{h^{\prime}}\end{array}$ & \\
\hline $5-19$ & $p-C z-B N C z$ & $466^{*}$ (in Tol) & $22^{*}$ (in Tol) & NA & NA & NA & NA & NA & 105 \\
\hline $5-20$ & $m-C z-B N C z^{i}$ & 519 (in Tol) & 38 (in Tol) & 97 (in Tol) & 520 & 44 & 27 & $(0.23,0.69)$ & \\
\hline $5-21$ & $2 \mathrm{~F}-\mathrm{BN} \mathrm{N}^{\mathrm{j}}$ & $\begin{array}{l}494 \text { (in Tol) } \\
502 \text { (in } \\
\text { mCPBC film) }\end{array}$ & $\begin{array}{l}24 \text { (in Tol) } \\
32 \text { (in mCPBC film) }\end{array}$ & 88.7 (in mCPBC film) & 501 & 40 & 22 & $(0.16,0.60)$ & 106 \\
\hline $5-22$ & $3 F-B N^{k}$ & $\begin{array}{l}499 \text { (in Tol) } \\
503 \text { (in } \\
\text { mCPBC film) }\end{array}$ & $\begin{array}{l}24 \text { (in Tol) } \\
33 \text { (in mCPBC film) }\end{array}$ & 83.4 (in mCPBC film) & 499 & 38.5 & 22.7 & $(0.20,0.58)$ & \\
\hline $5-23$ & $4 \mathrm{~F}-\mathrm{BN}^{\mathrm{k}}$ & $\begin{array}{l}496 \text { (in Tol) } \\
501 \text { (in } \\
\text { mCPBC film) }\end{array}$ & $\begin{array}{l}25 \text { (in Tol) } \\
31 \text { (in mCPBC film) }\end{array}$ & 91.4 (in mCPBC film) & 493 & 31.6 & 20.9 & $(0.12,0.48)$ & \\
\hline $5-24$ & AZA-BN & $\begin{array}{l}522 \text { (in Tol) } \\
526 \text { (in mCBP film) }\end{array}$ & $\begin{array}{l}28 \text { (in Tol) } \\
36 \text { (in mCBP film) }\end{array}$ & $\begin{array}{l}99.7 \text { (in Tol) } \\
94 \text { (in mCBP film) }\end{array}$ & $528^{l}, 527^{m}$ & $31^{\prime}, 30$ & $25.7^{1}, 28.2^{m}$ & $\begin{array}{l}(0.28,0.69)^{\prime} \\
(0.27,0.69)^{m}\end{array}$ & 107 \\
\hline $5-25$ & $\begin{array}{l}\text { CzDABNA-NP-M/ } \\
\text { TB }\end{array}$ & 468 (in PMMA fim) & 28 (in PMMA fim) & 86 (in PMMA fim) & NA & NA & NA & NA & 103 \\
\hline $5-26$ & $\begin{array}{l}\text { CZ2DABNA-NP- } \\
M / T B^{n}\end{array}$ & 478 (in PMMA fim) & 29 (in PMMA fim) & 85 (in PMMA fim) & 477 & 27 & 21.8 & $(0.11,0.23)$ & \\
\hline $5-27$ & CzB2-M/TB & 491 (in PMMA film) & 34 (in PMMA fim) & 88 (in PMMA fim) & NA & NA & NA & NA & \\
\hline $5-28$ & Cz2B2-M/TB & 483 (in PMMA film) & 38 (in PMMA fim) & 88 (in PMMA fim) & NA & NA & NA & NA & \\
\hline $5-29$ & CzDABNA-NP & 461 (in PMMA film) & 30 (in PMMA fim) & 80 (in PMMA fim) & NA & NA & NA & NA & \\
\hline $5-30$ & $\begin{array}{l}\text { CzDABNA-NP-TB/ } \\
\mathrm{H}\end{array}$ & 465 (in PMMA film) & 30 (in PMMA fim) & 82 (in PMMA fim) & NA & NA & NA & NA & \\
\hline $5-31$ & DABNA-NP-M & 460 (in PMMA film) & 29 (in PMMA fim) & 88 (in PMMA fim) & NA & NA & NA & NA & \\
\hline $5-32$ & DABNA-NP-TB ${ }^{n}$ & 453 (in PMMA film) & 26 (in PMMA fim) & 83 (in PMMA fim) & 457 & 33 & 19.5 & $(0.14,0.11)$ & \\
\hline $5-33$ & $\mathrm{CzB} 2-\mathrm{M} / \mathrm{P}^{\mathrm{n}}$ & 504 (in PMMA film) & 39 (in PMMA fim) & 87 (in PMMA fim) & 497 & 29 & 26.7 & $(0.12,0.57)$ & \\
\hline $5-34$ & $O A B-A B P-1{ }^{\circ}$ & 506 (in PMMA film) & 34 (in PMMA fim) & 90 (in PMMA film) & 505 & 33 & 21.8 & $(0.12,0.63)$ & 108 \\
\hline $5-35$ & $\mathrm{BBC}-\mathrm{DB} \mathrm{B}^{\mathrm{p}}$ & $\begin{array}{l}466 \text { (in Tol) } \\
471 \text { (in mCBP film) }\end{array}$ & $\begin{array}{l}16 \text { (in Tol) } \\
26 \text { (in mCBP film) }\end{array}$ & 93 (in Tol) & 469 & 27 & 29.3 & $(0.12,0.18)$ & 109 \\
\hline $5-36$ & $\mathrm{BBCz}-\mathrm{G}^{\mathrm{p}}$ & $\begin{array}{l}517 \text { (in Tol) } \\
519 \text { (in mCBP film) }\end{array}$ & $\begin{array}{l}34 \text { (in Tol) } \\
50 \text { (in mCBP film) }\end{array}$ & $\begin{array}{l}90 \text { (in Tol) } \\
99 \text { (in mCBP film) }\end{array}$ & 515 & 54 & 31.8 & $(0.26,0.68)$ & \\
\hline $5-37$ & $\mathrm{BBC} Z-Y^{p}$ & & & & 549 & 48 & 29.3 & NA & \\
\hline
\end{tabular}


Table 3 continued

\begin{tabular}{|c|c|c|c|c|c|c|c|c|c|}
\hline No. & Emitter & $\lambda_{\mathrm{PL}}(\mathrm{nm})$ & $\mathrm{FWHM}_{\mathrm{PL}}(\mathrm{nm})$ & PLQY (\%) & $\lambda_{\mathrm{EL}}(\mathrm{nm})$ & $\mathrm{FWHM}_{\mathrm{EL}}(\mathrm{nm})$ & EQE (\%) & $\mathrm{ClE}_{x, y}$ & Refs. \\
\hline $5-38$ & $\mathrm{BBC} z-\mathrm{R}^{\mathrm{p}}$ & $\begin{array}{l}549 \text { (in Tol) } \\
549 \text { (in mCBP film) } \\
615 \text { (in Tol) } \\
619 \text { (in mCBP film) }\end{array}$ & $\begin{array}{l}42 \text { (in Tol) } \\
48 \text { (in mCBP film) } \\
21 \text { (in Tol) } \\
27 \text { (in mCBP film) }\end{array}$ & $\begin{array}{l}85 \text { (in Tol) } \\
90 \text { (in mCBP film) } \\
89 \text { (in Tol) } \\
79 \text { (in mCBP film) }\end{array}$ & 616 & 26 & 22.0 & $(0.67,0.33)$ & \\
\hline $5-39$ & $\mathrm{QAO}(=\mathrm{QAD})^{\mathrm{q}}$ & 466 (in Tol) & 32 (in Tol) & 72.4 (in mCP film) & 468 & 39 & 19.4 & $(0.13,0.18)$ & 110 \\
\hline $5-40$ & 3-PhQAD ${ }^{r}$ & 466 (in Tol) & 30 (in Tol) & 73 (in mCP film) & 480 & 44 & 19.1 & $(0.13,0.32)$ & 111 \\
\hline $5-41$ & 7-PhQAD ${ }^{r}$ & 464 (in Tol) & 22 (in Tol) & 68 (in $\mathrm{mCP}$ film) & 472 & 34 & 18.7 & $(0.12,0.24)$ & \\
\hline $5-42$ & $\mathrm{Mes}_{3} \mathrm{DiKTa}^{\mathrm{s}}$ & $\begin{array}{l}468 \text { (in Tol) } \\
477 \text { (in mCP film) }\end{array}$ & $\begin{array}{l}29 \text { (in Tol) } \\
37 \text { (in } \mathrm{mCP} \text { film) }\end{array}$ & $\begin{array}{l}37 \text { (in Tol) } \\
80 \text { (in mCP film) }\end{array}$ & 480 & 36 & 21.1 & $(0.12,0.32)$ & 112 \\
\hline $5-43$ & $\mathrm{DDiKTa}^{\mathrm{t}}$ & $\begin{array}{l}470 \text { (in Tol) } \\
490 \text { (in DPEPO film) }\end{array}$ & $\begin{array}{l}47 \text { (in Tol) } \\
61 \text { (in DPEPO film) }\end{array}$ & $\begin{array}{l}29 \text { (in Tol) } \\
72 \text { (in DPEPO film) }\end{array}$ & 500 & 59 & 19.0 & $(0.18,0.53)$ & 113 \\
\hline $5-44$ & $\mathrm{QA}-\mathrm{PF}^{\mathrm{U}}$ & $\begin{array}{l}465 \text { (in Tol) } \\
478 \text { (in mCBP) }\end{array}$ & $\begin{array}{l}23 \text { (in Tol) } \\
30 \text { (in mCBP fim) }\end{array}$ & $\begin{array}{l}73 \text { (in Tol) } \\
89 \text { (in mCBP fim) }\end{array}$ & 474 & 27 & 16.8 & $(0.12,0.17)$ & 114 \\
\hline $5-45$ & QA-PCN ${ }^{u}$ & $\begin{array}{l}462 \text { (in Tol) } \\
477 \text { (in mCBP) }\end{array}$ & $\begin{array}{l}25 \text { (in Tol) } \\
34 \text { (in mCBP fim) }\end{array}$ & $\begin{array}{l}42 \text { (in Tol) } \\
68 \text { (in mCBP fim) }\end{array}$ & 473 & 30 & 16.9 & $(0.12,0.18)$ & \\
\hline $5-46$ & $\mathrm{QA}-\mathrm{PMO}^{\mathrm{u}}$ & $\begin{array}{l}475 \text { (in Tol) } \\
485 \text { (in mCBP) }\end{array}$ & $\begin{array}{l}27 \text { (in Tol) } \\
33 \text { (in mCBP fim) }\end{array}$ & $\begin{array}{l}75 \text { (in Tol) } \\
66 \text { (in mCBP fim) }\end{array}$ & 484 & 27 & 15.0 & $(0.11,0.30)$ & \\
\hline $5-47$ & $\mathrm{QA}-\mathrm{PCZ} \mathrm{Z}^{\mathrm{u}}$ & $\begin{array}{l}471 \text { (in Tol) } \\
480 \text { (in mCBP) }\end{array}$ & $\begin{array}{l}29 \text { (in Tol) } \\
34 \text { (in mCBP fim) }\end{array}$ & $\begin{array}{l}54 \text { (in Tol) } \\
71 \text { (in mCBP fim) }\end{array}$ & 482 & 29 & 17.5 & $(0.11,0.28)$ & \\
\hline \multicolumn{10}{|c|}{ Conventional D-A type TADF emitters } \\
\hline $5-48$ & $\mathrm{CZBPCN}^{\vee}$ & 453 (in Tol) & 55 (in Tol) & 76 (in Tol) & 460 & 48 & 14.0 & $(0.14,0.12)$ & \\
\hline $5-49$ & TDBA-AC & 458 (in Tol) & 50 (in Tol) & 93 (in DBFPO film) & $445^{*, w}, 464^{*, x}$ & $48^{w}, 55^{x}$ & $21.5^{w}, 25.7^{x}$ & $\begin{array}{l}(0.15,0.06)^{w} \\
(0.14,0.15)^{x}\end{array}$ & 116 \\
\hline $5-50$ & TDBA-DI & 456 (in Tol) & 55 (in Tol) & 99 (in DBFPO film) & $462^{*, w}, 480^{*, x}$ & $56^{w}, 65^{x}$ & $32.2^{w}, 38.2$ & $\begin{array}{l}(0.14,0.15)^{w} \\
(0.15,0.28)^{x^{\prime}}\end{array}$ & \\
\hline $5-51$ & $3 C z \operatorname{TB}^{x}$ & 433 (in Tol) & $50^{*}$ (in Tol) & 87.8 (in DBFPO film) & 470 & $56^{*}$ & 29.1 & $(0.14,0.19)$ & 117 \\
\hline $5-52$ & $\mathrm{M} 3 \mathrm{CzB}^{\mathrm{x}}$ & 445 (in Tol) & $53^{*}$ (in Tol) & 92.8 (in DBFPO film) & 478 & $54^{*}$ & 30.7 & $(0.14,0.26)$ & \\
\hline
\end{tabular}

EtOH ethanol, mCBP: 3,3'-di(9H-carbazol-9-yl)-1,1'-biphenyl, mCPBC: 9-(3-(9H-carbazol-9-yl) phenyl)-9H-3,9'-bicarbazole, DOBNA-OAr 7-((2'-methyl-[1,1'-biphenyl]-3-yl) oxy)-3,11-di-o-tolyl-5,9-dioxa-13b-boranaphtho[3,2,1-de]anthracene, DMAC-DPS 10,10'-(4,4'-sulfonylbis(4,1-phenylene))bis(9,9-dimethyl-9,10-dihydroacridine, 2,6DCzppy 2,6-bis(3-(carbazol-9-yl)phenyl))pyridine, PIM-TRZ 2-(3-(4,6-diphenyl-1,3,5-triazin-2-yl)phenyl)-1-phenyl-1H-benzo[d]imidazole, $P h C z B C Z$ 9-(2-(9-phenyl-9Hcarbazol-3-yl)phenyl)-9H-3,9'-bicarbazole, $B C z P h$ 9,9'-diphenyl-9H,9' $\mathrm{H}$-3,3'-bicarbazole, CzPhPy 4,6-bis(3-(9H-carbazol-9-yl)phenyl)pyrimidine, 3,4-2CzBN: 3,4-di(9Hcarbazol-9-yl)benzonitrile, BPy-TP2 2,7-di([2,2'-bipyridin]-5-yl)triphenylene, Plexcore OC AQ-1200 poly(thiophene-3-[2[(2-methoxyethoxy)ethoxy]]-2,5-diyl), PPF 2,8-bis (diphenylphosphoryl)dibenzo[b,d]-furan, B3PyPB 1,3-bis[3,5-di(pyridin-3-yl)phenyl]-benzene, $P P B I$ 5-(5-(2,4,6-triiso-propylphenyl)pyridin-2-yl)-5H-benzo[d]benzo[4,5] imidazo[1,2-a]imidazole, B4PyPPm 4,6-bis(3,5-di(pyridin-4-yl)phenyl)-2-phenylpyrimidine, 5TCzBN 2,3,4,5,6-pentakis-(3,6-di-tert-butyl-9H-carbazol-9-yl) benzonitrile.

*Estimated values based on the graph (not provided by author).

aITO/NPD/TCTA/mCP/mCBP:1 wt\% emitter/TSPO1/LiF/Al.

bITO/HATCN/NPD/TCTA/mCP/DOBNA-OAr:1 wt\% emitter/TSPO1/LiF/Al.

CITO/NPD/TCTA/mCP/DOBNA-OAr:1 wt\% emitter/TSPO1/LiF/Al.

dITO/PEDOT:PSS/TAPC/mCP/DPEPO:30 wt\% DMAC-DPS:1 wt\% emitter/TSPO1/TPBi/LiF/AI

eITO/MoO $/$ /TAPC/2,6-DCzppy:4 wt\% emitter/TmPyPB/LiF/Al.

fITO/TAPC/TCTA/mCBP:1 wt\% emitter/TmPyPB/LiF/Al.

ITO/TAPC/TCTA/TCTA:PIM-TRZ (1:2):1 wt\% emitter/TmPyPB/LiF/AI.

hITO/TAPC/TCTA/TCTA:PIM-TRZ (1:2):2 wt\% emitter/TmPyPB/LiF/Al.

IITO/TAPC/TCTA/PhCzBCz:3 wt\% emitter/TmPyPB/LiF/Al.

j|TO/HATCN/NPB/BCzPh/mCPBC:35 wt\% 5TCzBN:9 wt\% emitter/9Cz46Pm/DPPyA:Liq (1:1)/LiF/Al.

k ITO/HATCN/NPB/BCzPh/mCPBC:35 wt\% 5TCzBN:6 wt\% emitter/9Cz46Pm/DPPyA:Liq (1:1)/LiF/Al.

ITO/ $\mathrm{MoO}_{3} / \mathrm{TAPC} / \mathrm{mCBP}: 4$ wt\% emitter/TmPyPB/LiF/Al.

mITO/HATCN/NPB/BCzPh/mCBP:30 wt\% Ir(ppy)3:6 wt\% emitter/CzPhPy/DPPyA/LiF/Al.

nITO/NPD/TCTA/mCP/DOBNA-Tol:1 wt\% emitter/3,4-2CzBN/BPy-TP2/LiF/Al.

OITO/Plexcore OC AQ-1200/Polymer A/Polymer B:2 wt\% emitter/NaF/Al.

PITO/HATCN/TAPC/mCBP/mCBP:2 wt\% emitter/PPF/B3PyPB/Liq/Al.

qITO/HATCN/TAPC/TCTA/mCP:5 wt\% emitter/B4PyPPm/Liq/Al.

'ITO/TAPC/TCTA/mCP:2 wt\% emitter/TmPyPB/LiF/AI.

sITO/HATCN/TAPC/TCTA/mCP:3.5 wt\% emitter/TmPyPB/LiF/Al.

tITO/TCTA/CzSi/DPEPO:9 wt\% emitter/TmPyPB/LiF/Al.

uITO/HATCN/TAPC/TCTA/mCP:3 wt\% emitter/TmPyPb/LiF/AI.

vITO/PEDOT:PSS/TAPC/mCP/DPEPO:5 wt\% emitter/TSPO1/TPBi/LiF/AI.

${ }^{w}$ ITO/HATCN/TAPC/DCDPA/PPBI:20 wt\% emitter/DBFPO/TPBi/LiF/AI.

xITO/HATCN/TAPC/DCDPA/DBFPO:20 wt\% emitter/DBFPO/TPBi/LiF/Al.

roll-off was still observed for the boron-containing ADBNAMe-Tip-based device, the EQE was sufficiently high (15.4\%) even at high luminance $\left(100 \mathrm{~cd} \mathrm{~m}^{-2}\right)$. In 2019, Hatakeyama developed a new pseudo-linear TADF molecule, $\left(N^{7}, N^{7}, N^{13}\right.$, $N^{13}, 5,9,11,15$-octaphenyl-5,9,11,15-tetrahydro-5,9,11,15tetraaza-19b,20b-diboradinaphtho[3,2,1-de:1',2',3'-jk]pentacene-7,13-diamine) ( $v$-DABNA, 5-14) ${ }^{95}$. Through the electronic interaction of two boron atoms and six nitrogen atoms, this compound exhibited a well-distributed HOMO and LUMO and showed narrow PL emission with $\mathrm{FWHM}=14 \mathrm{~nm}$ at $468 \mathrm{~nm}$ with $74 \%$ PLQY in toluene. In addition, remarkably reduced vibronic coupling was observed in the PL emission of $v$-DABNA compared to that of the fluorescent emitter perylene 


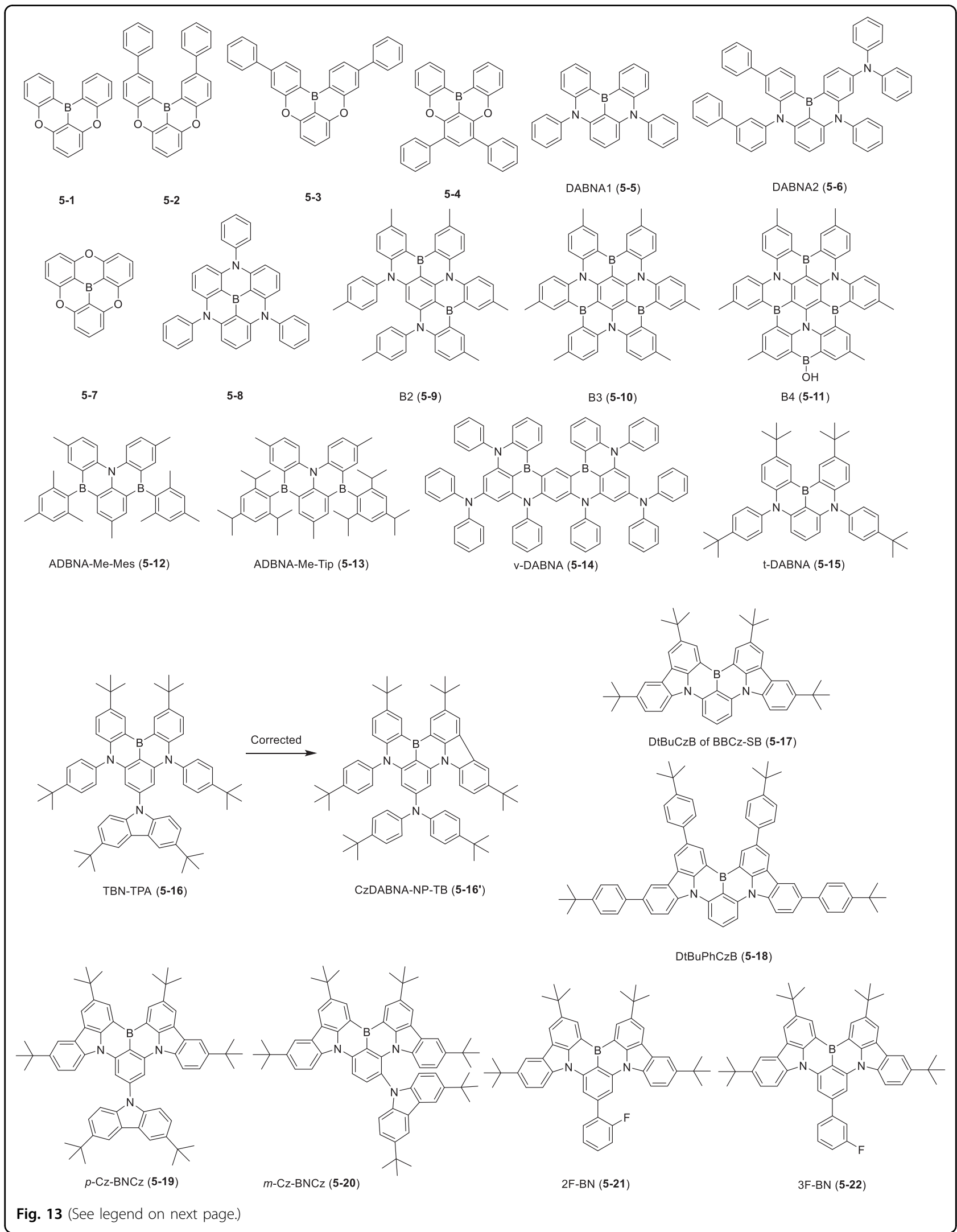



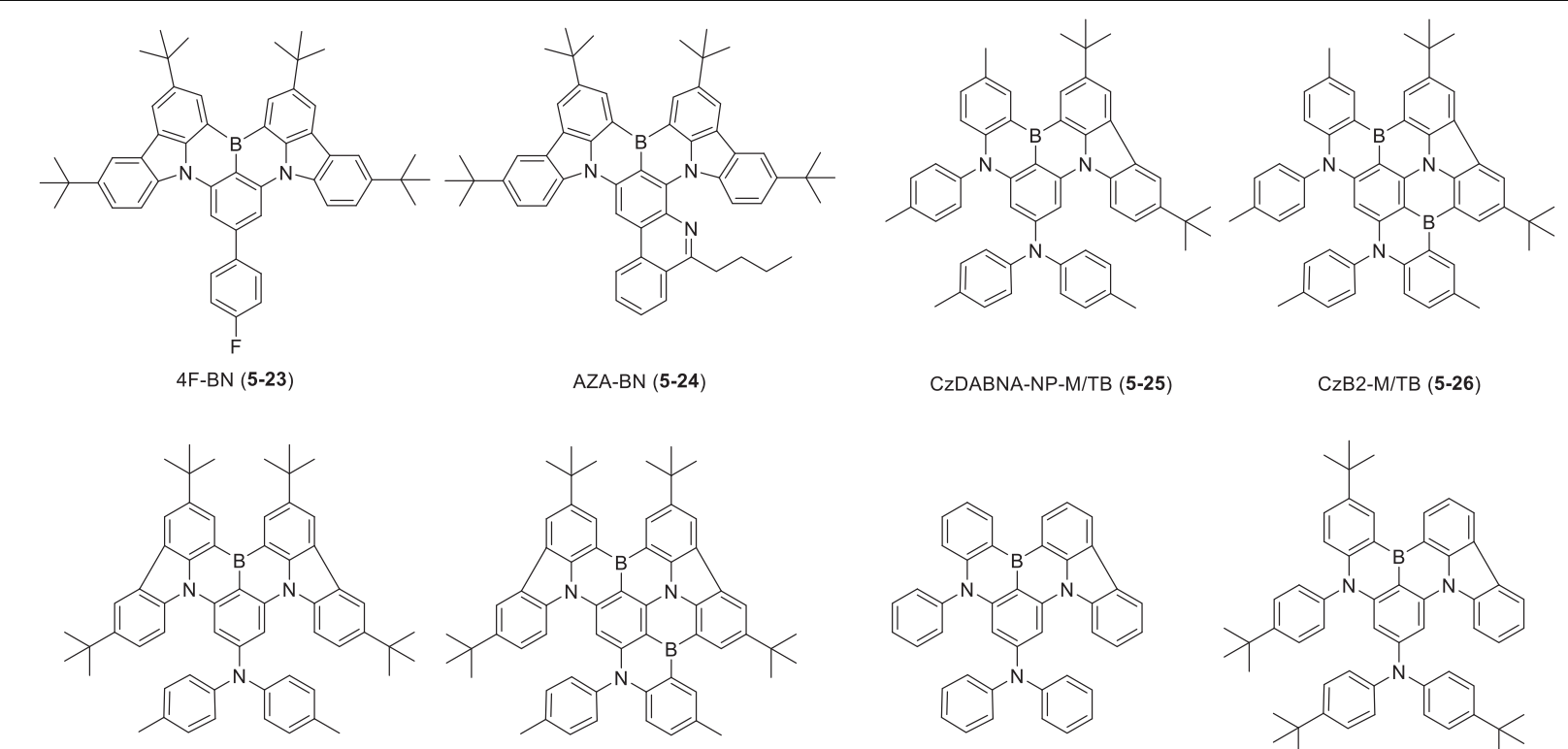

Cz2DABNA-NP-M/TB (5-27)

Cz2B2-M/TB (5-28)

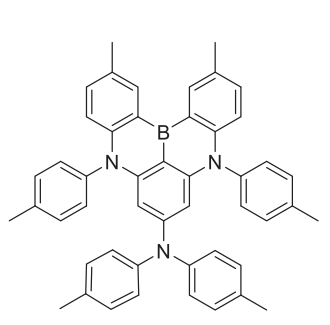

DABNA-NP-M (5-31)

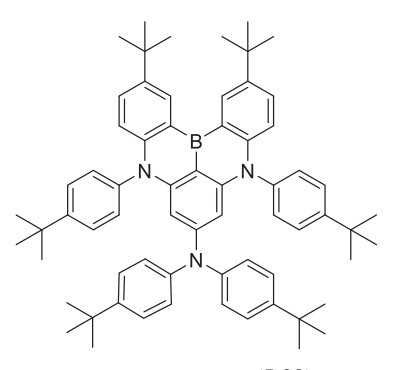

$R=\xi-<$

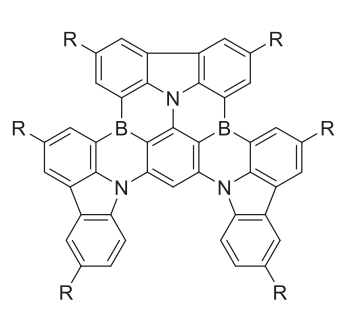

BBCz-DB (5-35)

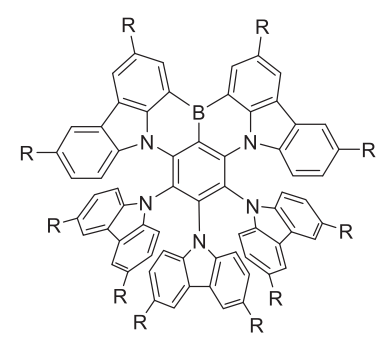

$\mathrm{BBC} z-\mathrm{G}(5-36)$

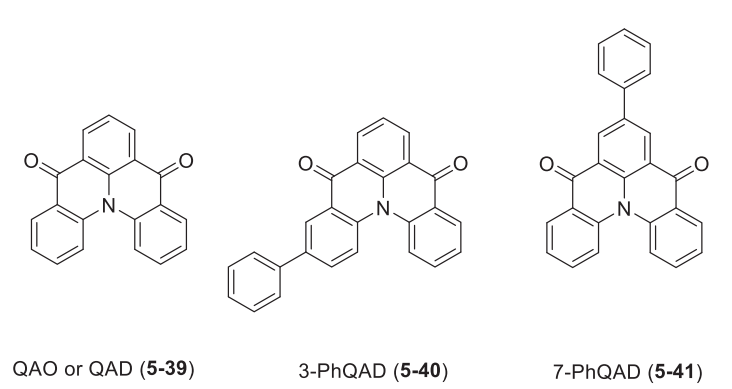

CzDABNA-NP (5-29)

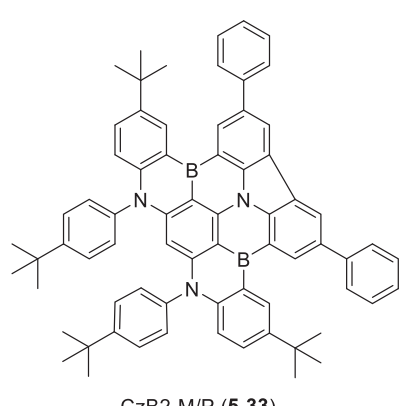

CzB2-M/P (5-33)

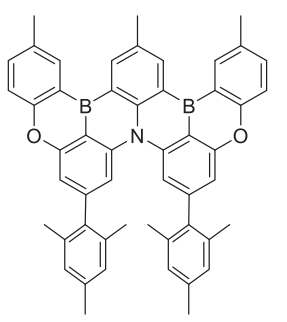

OAB-ABP-1 (5-34)

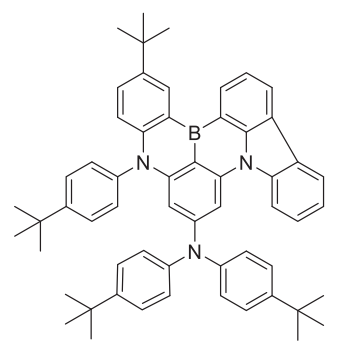

CzDABNA-NP-TB/H (5-30)

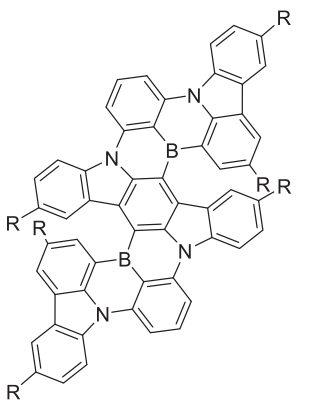

$\mathrm{BBC}-\mathrm{R}(\mathbf{5 - 3 8 )}$

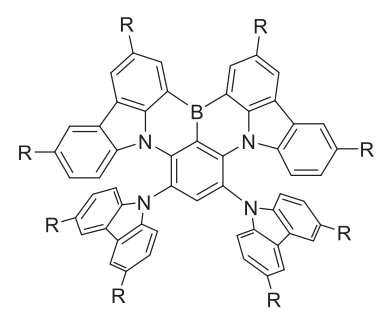

$B B C Z-Y(5-37)$

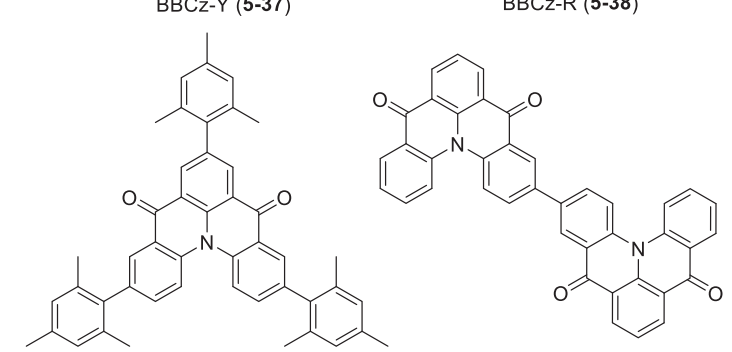

$\mathrm{Mes}_{3}$ DiKTa (5-42)

DDiKTa (5-43)

Fig. 13 (See legend on next page.) 

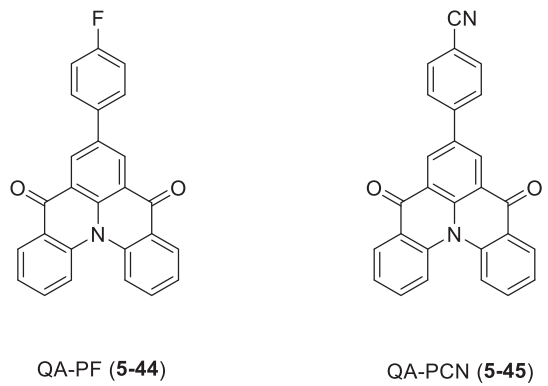

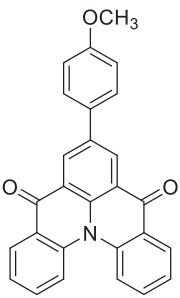

QA-PMO (5-46)

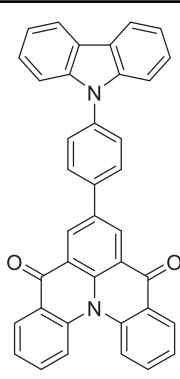

QA-PCZ (5-47)

Fig. 13 Chemical structures of narrow-bandwidth MR-TADF emitters.

due to the MR effect (Fig. 14d). Employing an MR-TADFtype host (DOBNA-OAr) based on structure 5-1 caused the PLQY to improve remarkably up to $90 \%$ $(\mathrm{FWHM}=18 \mathrm{~nm})$. The OLEDs fabricated with $v$-DABNA (ITO/NPD/TCTA/mCP/DOBNA-OAr:1 wt\% v-DABNA/ TSPO1/LiF/Al) achieved a sharp blue emission at $469 \mathrm{~nm}$ with $\mathrm{FWHM}=18 \mathrm{~nm}$ (Fig. 14e), which is comparable to that of QDs or perovskite-LEDs. The v-DABNA-based blue OLED exhibited not only the smallest FWHM but also the highest EQE (34.4\%) among the MR-TADF emitters reported thus far owing to its small $\Delta E_{\mathrm{ST}}$ $(0.017 \mathrm{eV})$ and efficient RISC.

The $\mathrm{B} / \mathrm{N}$ frameworks designed by Hatakeyama have been extensively modified by other research groups. Lee et al. newly synthesized 2,12-di-tert-butyl-5,9-bis(4-(tert-butyl) phenyl)-5,9-dihydro-5,9-diaza-13b-boranaphtho[3,2,1-de] anthracene ( $t$-DABNA, 5-15) by modifying DABNA1 with tert-butyl groups to hinder intermolecular packing ${ }^{101}$. In this study, they reported a ternary emitting system based on the TADF-assisted TADF (TATADF) strategy by incorporating an additional TADF assistant dopant. To fabricate the TATADF device (ITO/PEDOT:PSS/TAPC/mCP/tDABNA/TSPO1/TPBi/LiF/Al), a TADF assistant dopant (DMAC-DPS) was introduced into the emitting layer (1 wt\% $t$-DABNA in DPEPO) to decrease the PL lifetime and improve $k_{\text {RISC }}$ via efficient upconversion. The fabricated $t-$ DABNA-based TATADF device showed a narrow EL emission at $450 \mathrm{~nm}$ (FWHM of $31 \mathrm{~nm}$ ) and a remarkably high EQE of 31.4\% (Fig. 14f). Huang et al. synthesized a new MR-TADF molecule, 2,12-di-tert-butyl-5,9-bis(4-(tert-butyl) phenyl)-7-(3,6-di-tert-butyl-9H-carbazol-9-yl)-5,9-dihydro5,9-diaza-13b-boranaphtho[3,2,1-de]anthracene, by introducing $t$-butyl substituents and a carbazole moiety to the DABNA1 structure (TBN-TPA, 5-16; the molecular structure was corrected to $5-1 \mathbf{6}^{\prime}$ by Hatakeyama based on its ${ }^{1} \mathrm{H}$ NMR spectrum) ${ }^{102,103}$. The electron-donating group at the para-position relative to boron enhanced the oscillator strength, achieving a high PLQY (97.48\%) in toluene. A narrow PL emission at $470 \mathrm{~nm}$ (FWHM $26 \mathrm{~nm}$ ) and extremely small Stokes shift $(10 \mathrm{~nm})$ were measured; these were attributed to the structural rigidity of the molecule. A similar EL spectrum was also observed at $474 \mathrm{~nm}$ with $\mathrm{FWHM}=27 \mathrm{~nm}$ and high EQE up to $32.1 \%$.

Narrowband green-emissive MR-TADF emitters have also been developed by extending the conjugation of boroncontaining rigid molecular backbones. Wang et al. reported two types of MR-TADF molecules, sky blue-emitting $\mathrm{DtBuCzB}(5-17)$ and green-emitting $\mathrm{DtBuPhCzB}$ (5-18), based on the dicarbazolylphenylene framework ${ }^{104}$. In toluene, $\mathrm{DtBuCzB}$ and $\mathrm{DtBuPhCzB}$ exhibited PL spectra at 481 and $496 \mathrm{~nm}$ with FWHMs of 22 and $21 \mathrm{~nm}$, respectively. The extended conjugation (compared to the blueemissive MR-TADF structures discussed above) causes a bathochromic shift in the emission, but a small FWHM was maintained owing to their structural rigidity, with a small Stokes shift of 14-15 nm for both compounds. With the device structure of ITO/TAPC/TCTA/mCBP:1 wt\% $\mathrm{DtBuCzB}$ or $\mathrm{DtBuPhCzB} / \mathrm{TmPyPB} / \mathrm{LiF} / \mathrm{Al}$, EL devices based on $\mathrm{DtBuCzB}$ and $\mathrm{DtBuPhCzB}$ showed narrow EL spectra (FWHM $29 \mathrm{~nm}$ ) at 488 and $504 \mathrm{~nm}$, respectively. To achieve a further shift in the emission maximum to a longer wavelength, the $\mathrm{DtBuCzB}$ core was modified by incorporating ditert-butylcarbazole into the para- or meta-positions relative to the boron atom, yielding $p-\mathrm{Cz}-\mathrm{BNCz}(5-19)$ and $m-\mathrm{Cz}-$ $\mathrm{BNCz}$ (5-20), respectively (Fig. 15a) ${ }^{105}$. In $p$-Cz-BNCz, an identical $\mathrm{HOMO} / \mathrm{LUMO}$ distribution and similar optical properties to those of $\mathrm{DtBuCzB}$ were observed (Fig. 15b). In contrast, the meta-substituted carbazole moiety in $m-\mathrm{Cz}-$ $\mathrm{BNCz}$ upshifted the $\mathrm{HOMO}$ with a decreased bandgap to yield green emission. Because of the twisted structure of the meta-substituted carbazole and boron-substituted phenyl ring in $m-\mathrm{Cz}-\mathrm{BNCz}$, a narrow PL was observed with $\mathrm{FWHM}=38 \mathrm{~nm}$ and without ICT character. Devices fabricated with different concentrations of $m-\mathrm{Cz}-\mathrm{BNCz}$ (1-50 wt\%) exhibited similar green EL spectra at $520-528 \mathrm{~nm}$ with small FWHM (all $<48 \mathrm{~nm}$ ) (Fig. 15c). Weak intermolecular interactions caused by structural distortion helped to achieve a high EQE (31\%), even with dopant concentrations of $10 \mathrm{wt} \%$. In particular, the device with $3 \mathrm{wt} \% \mathrm{~m}-\mathrm{Cz}-\mathrm{BNCz}$ achieved exceptionally pure green 
a

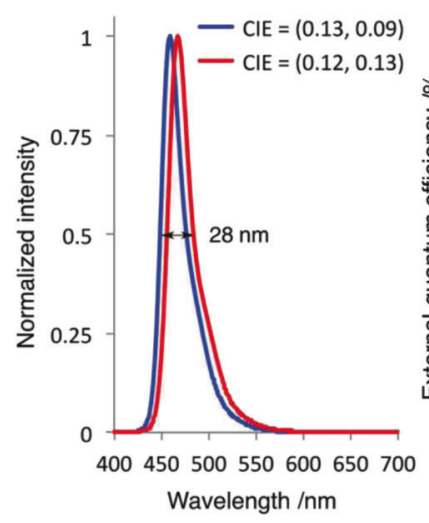

d
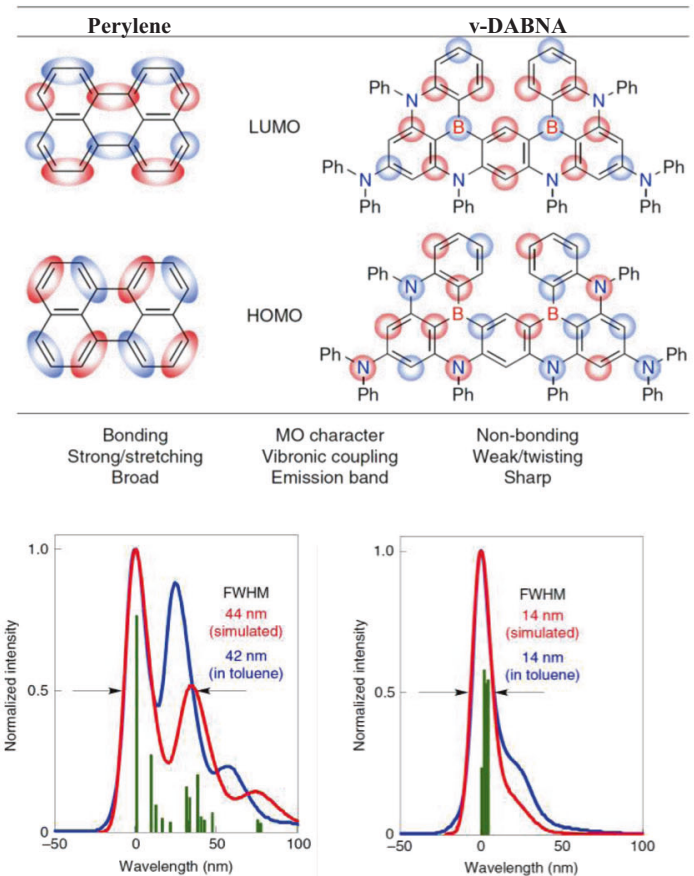

b

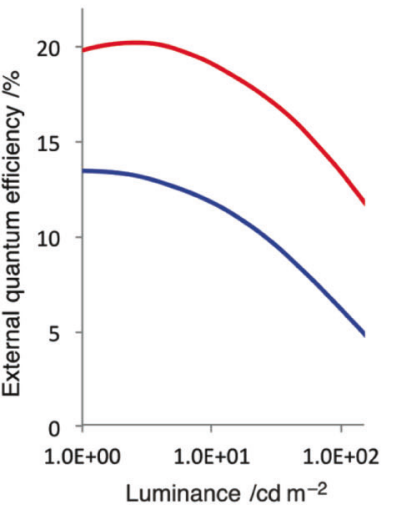

C

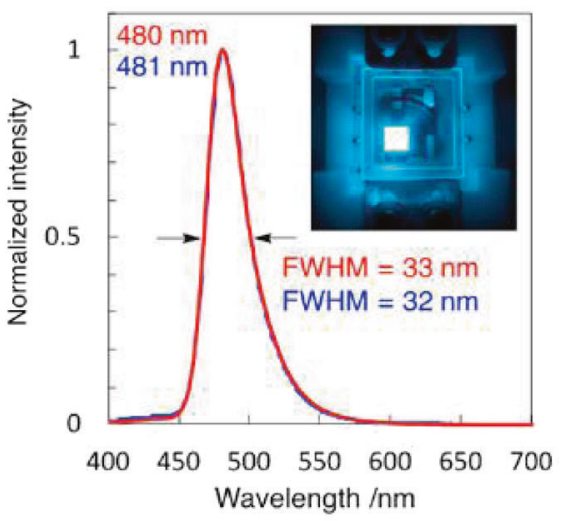

e
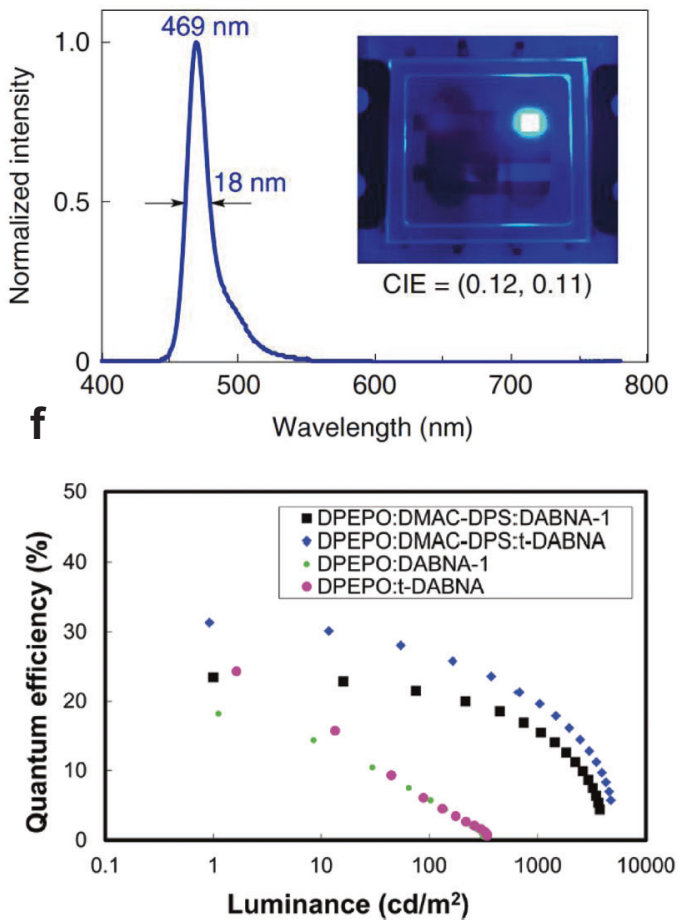

Fig. 14 Light-emitting characteristics of blue MR-based TADF emitters. a EL spectra and $\mathbf{b}$ EQE luminance characteristics of OLEDs fabricated with DABNA1 (blue) and DABNA2 (red). Reproduced with permission ${ }^{94}$. Copyright 2016, Wiley-VCH. c Comparison of EL spectra of ADBNA-Me-Mes (blue) and ADBNA-Me-Tip (red). Reproduced with permission ${ }^{100}$. Copyright 2019, American Chemical Society. d HOMO/LUMO distribution and corresponding emission spectra of perylene and v-DABNA. e EL spectrum of v-DABNA-based OLED. Reproduced with permission ${ }^{95}$. Copyright 2019, Springer Nature. $\mathrm{f}$ EQE luminance characteristics of devices fabricated with t-DABNA or DABNA1. Reproduced with permission ${ }^{101}$. Copyright 2019 , the Royal Society of Chemistry.

emission with CIE coordinates of $(0.23,0.69)$. Another strategy to induce green emission is the introduction of an electron-withdrawing moiety onto the para-position with respect to boron in $\mathrm{DtBuCzB}$. By introducing a fluorinesubstituted phenyl unit, Duan et al. synthesized a series of green-emissive TADF emitters, 2F-BN (5-21), 3F-BN (5-22), and 4F-BN $(5-23)^{106}$. When these emitters were doped into a 9-(3-(9H-carbazol-9-yl)phenyl)-9H-3,9'-bicarbazole $(\mathrm{mCPBC})$ film, emission maxima were observed at $501 \sim 503 \mathrm{~nm}$ with FWHM = 31 33 nm and high PLQYs of $\sim 90 \%$, regardless of the positions of the fluorine substituents. With a device architecture of ITO/HAT-CN/NPB/BCzPh/ 2F-, 3F-, or 4F-BN/9Cz46Pm/DPPyA:Liq (1:1)/LiF/Al, narrowband green EL spectra were observed at 501, 499 and 
a
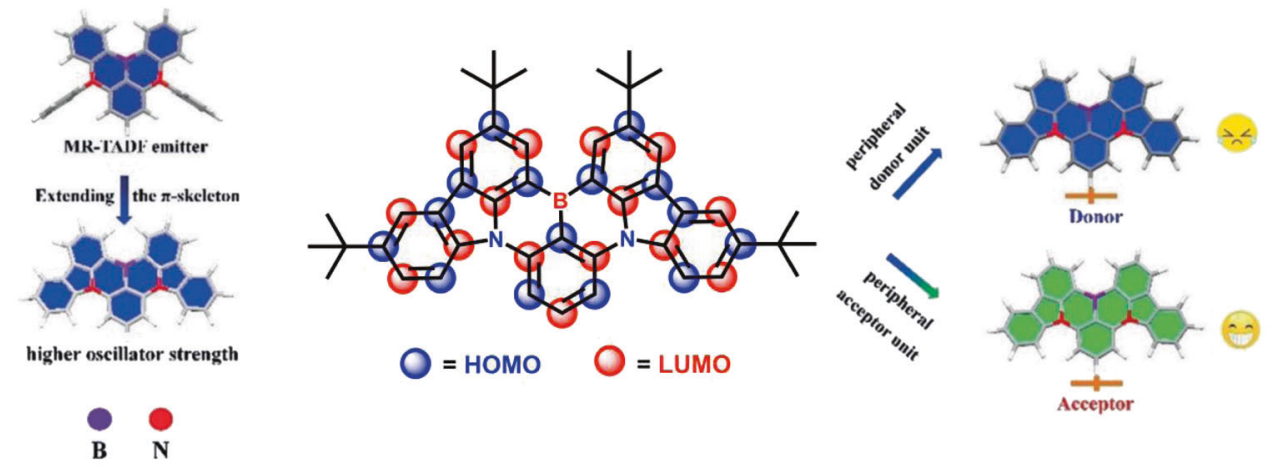

b

DtBuCz

LUMO

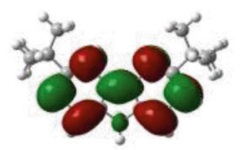

$-0.58 \mathrm{eV}$

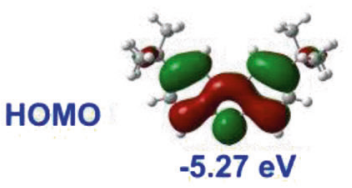

Egap

$4.69 \mathrm{eV}$

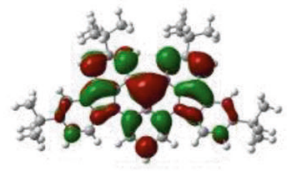

$-1.71 \mathrm{eV}$

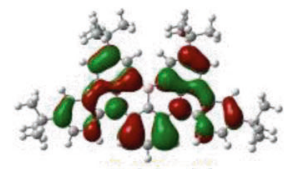

$-5.06 \mathrm{eV}$

$3.35 \mathrm{eV}$
p-Cz-BNCz

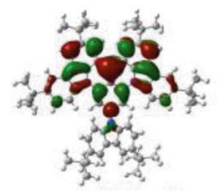

$-1.79 \mathrm{eV}$

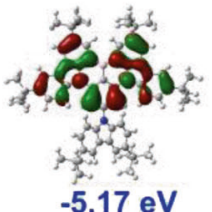

$3.38 \mathrm{eV}$

\section{$m-\mathrm{Cz}-\mathrm{BNCz}$}

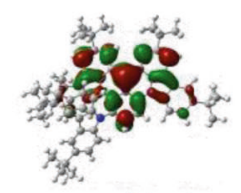

$$
-1.82 \mathrm{eV}
$$

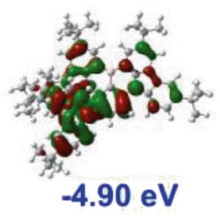

$3.08 \mathrm{eV}$

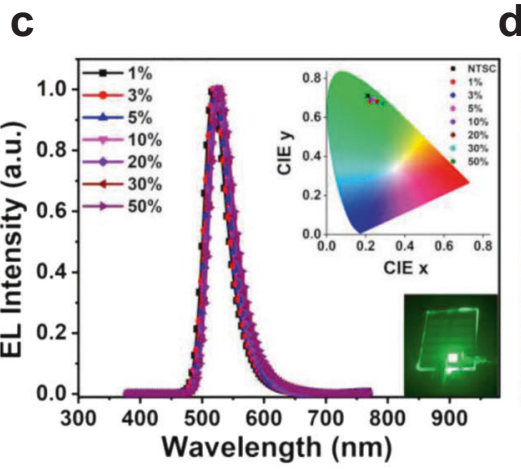

d

e

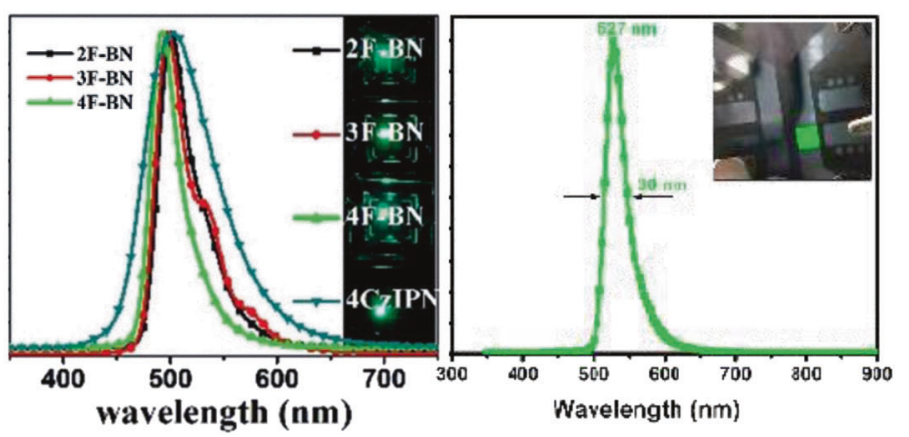

Fig. 15 Electronic structures and light-emitting properties of green MR-TADF emitters. a Structural modification of green-emissive DABNA derivatives. $\mathbf{b} \mathrm{HOMO} / \mathrm{LUMO}$ distributions of DtBuCz, DtBuCzB, p-Cz-BNCz, and m-Cz-BNCz. c-e EL spectra (inset: photographs of their green-emissive OLEDs) of $\mathbf{c} m-C z-B N C z, \mathbf{d} 2 F-B N, 3 F-B N, 4 F-B N$, and e AZA-BN. a (middle), b, c Reproduced with permission ${ }^{105}$. Copyright 2020, Wiley-VCH. a (left and right figures) and $\mathbf{d}$ Reproduced with permission ${ }^{106}$. Copyright 2019, Wiley-VCH. e Reproduced with permission ${ }^{107}$. Copyright 2020 , Wiley-VCH.

$493 \mathrm{~nm}$ (with FWHM of 40, 38 and $31 \mathrm{~nm}$ ) for 2F-BN, 3F$\mathrm{BN}$, and $4 \mathrm{~F}-\mathrm{BN}$, respectively. Duan also developed another type of B/N framework, 8,11,14,17-tetra-tert-butyl-21-butyl5b,19b,20-triaza-12b-borabenzo[a]fluorantheno[1,2,3-fg] indeno[1,2,3-mn]tetracene (AZA-BN, 5-24), which has extended conjugation with isoquinoline fused to the central benzene ring ${ }^{107}$. The PL spectrum of the $4 \mathrm{wt} \%$ AZA-BNdoped mCBP film showed pure green emission at $\lambda_{\mathrm{PL}}=$ $526 \mathrm{~nm}$ with a small FWHM $(36 \mathrm{~nm})$ and a high PLQY
(94\%). In particular, when AZA-BN was utilized as a phosphorescence-sensitized TADF dopant in iridium-based PhOLEDs, a narrowband EL spectrum at $527 \mathrm{~nm}$ (FWHM $30 \mathrm{~nm}$ ) was obtained with high EQE (28.2\%) and color purity with CIE coordinates of $(0.27,0.69)$.

Hatakeyama also reported a series of carbazole-based MRTADF DABNA analogs (5-25 5-33) via regioselective oneshot borylation (Fig. 16a) ${ }^{103}$. Among them, CzB2-M/P showed a green PL emission at $504 \mathrm{~nm}$ in a PMMA film with 


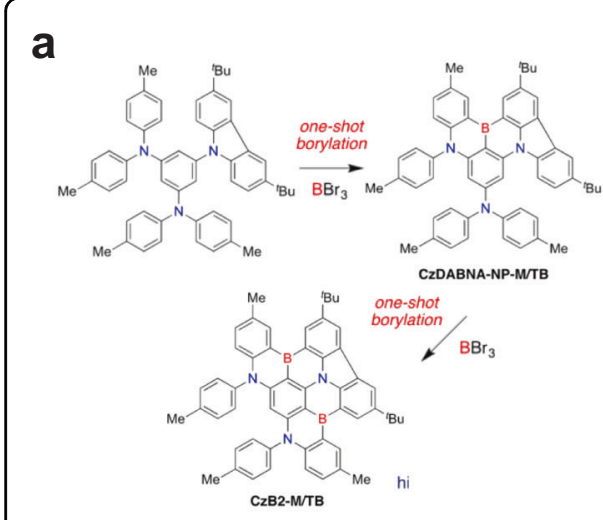

d

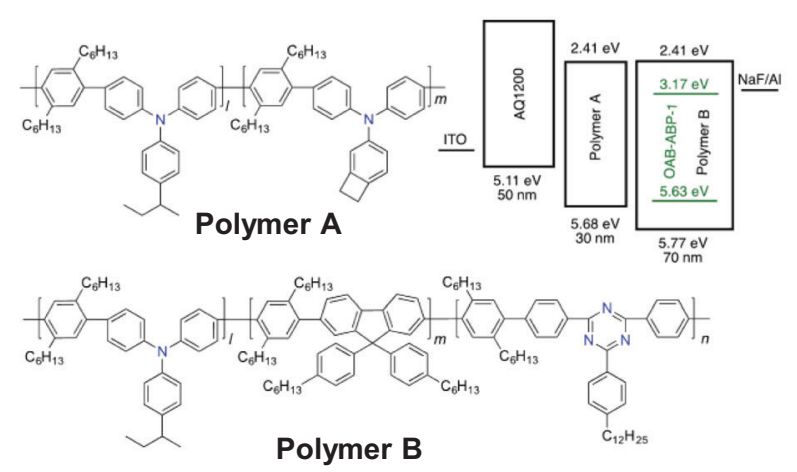

f

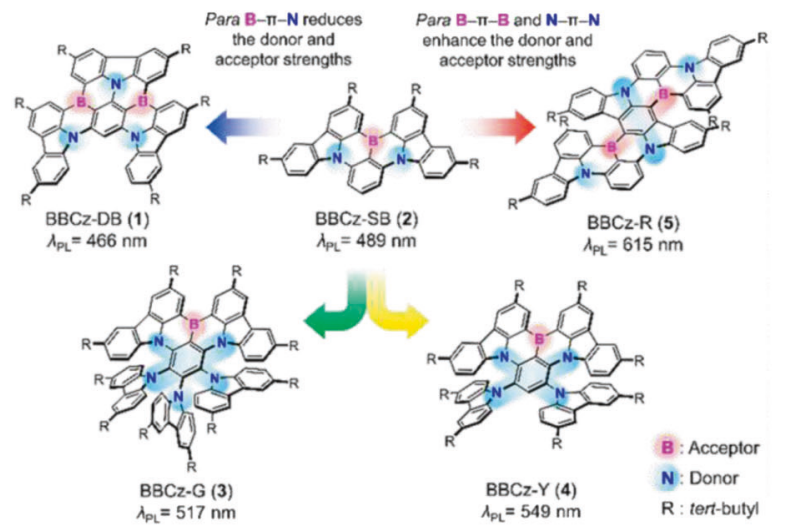

b

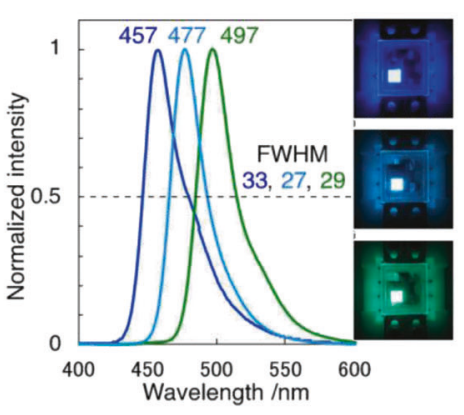

C

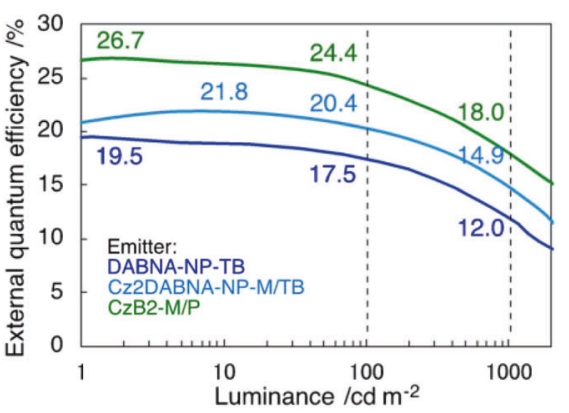

e

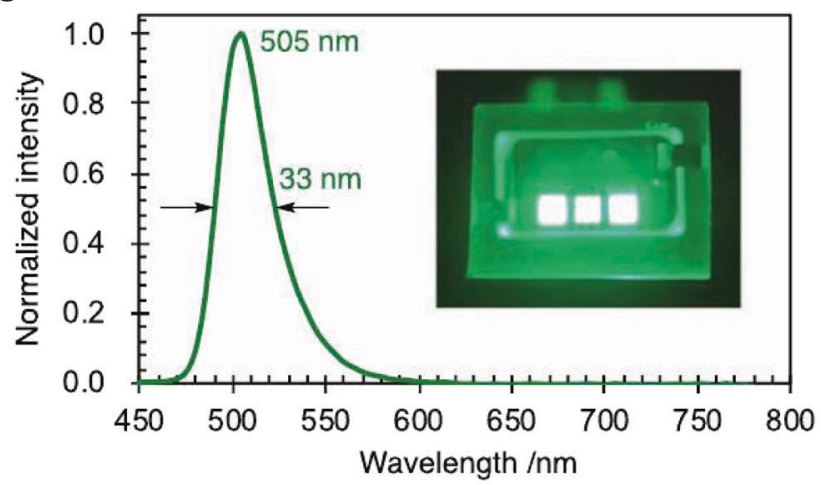

g

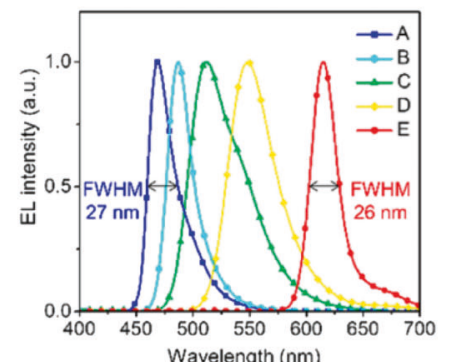

h
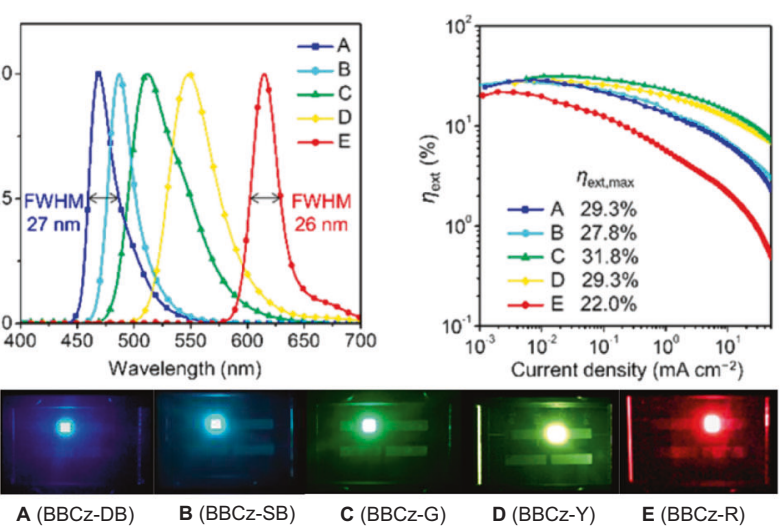

Fig. 16 MR-TADF emitters with expanded emission colors. a Scheme of sequential borylation to yield CzDABNA-NP-M/TB and CzB2-M/TB. b EL spectra of OLEDs employing DABNA-NP-TB, CzDABNA-NP-M/TB, CZB2-M/P and $\mathbf{c}$ plots of their EQE versus luminance. $\mathbf{d}$ Molecular structure of polymers and device configuration of OLEDs employing OAB-ABP-1 as a green emitter, and e EL spectrum. f Molecular design strategies for full-color MR-TADF emitters (BBCz-DB, BBCz-G, BBCz-Y, BBCz-R), $\mathbf{g}$ EL spectra, and $\mathbf{h}$ EQE plots. a-c Reproduced with permission ${ }^{103}$. Copyright 2020, Wiley-VCH. d, e Reproduced with permission ${ }^{108}$. Copyright 2020, Wiley-VCH. f-h Reprinted with permission ${ }^{109}$. Copyright 2020, American Chemical Society.

a high PLQY (87\%), and its EL device exhibited a narrow emission at $497 \mathrm{~nm}$ (FWHM of $29 \mathrm{~nm}$ ) (Fig. 16b) with a maximum EQE of $26.7 \%$ (Fig. 16c) and a long operational lifetime of $74 \mathrm{~h}$. They also designed another solutionprocessable green-emitting MR-TADF material, OAB-ABP$1(5-34)^{108}$. By delicate modulation of HOMO and LUMO separation, the oscillator strength of OAB-ABP-1 was sufficiently high $(f=0.3231)$, with minimized vibronic coupling in the $\mathrm{S}_{0}-\mathrm{S}_{1}$ transitions. As a result, a narrow green PL peak with a small FWHM was observed at $506 \mathrm{~nm}(34 \mathrm{~nm})$ in the PMMA film, and the solution-processed OLED device using a bipolar polymer host (Fig. 16d) was demonstrated to successfully show a narrow EL spectrum at $505 \mathrm{~nm}$ with $\mathrm{FWHM}=33 \mathrm{~nm}$ (Fig. 16e). 
Recently, Yasuda et al. reported four different kinds of MR-TADF molecules based on B/N-fused PAH structures (5-35 5-38) covering full-color emissions in the visible range ${ }^{109}$. Importantly, $\mathrm{BBCz}-\mathrm{R}$ successfully demonstrated MR-TADF-based red emission for the first time. By differentiating the substituent positions of $B$ and $\mathrm{N}$ atoms, the donating and accepting abilities were finely controlled. For instance, the donor strength of the $\mathrm{N}$ atoms bound in central benzene is depressed in $\mathrm{BBCz}-\mathrm{DB}$ due to the para-positioned $\mathrm{B}$ atom, leading to deeper blue emission $(466 \mathrm{~nm})$ in toluene compared to that of $\mathrm{BBCz}-\mathrm{SB}(481 \mathrm{~nm})$ (Fig. 16f). In contrast, the symmetrical substitution of $\mathrm{N}$ or $\mathrm{B}$ atoms in $\mathrm{BBCz}-\mathrm{R}$ strengthens the polar effect, leading to a bathochromic shift in the PL emission. The OLED devices fabricated with BBCz-DBA, $\mathrm{BBCz}-\mathrm{G}, \mathrm{BBCz}-\mathrm{Y}$, and $\mathrm{BBCz}-\mathrm{R}$ exhibited narrowband and full-color EL spectra $\left(\lambda_{\mathrm{EL}}=469,515,549\right.$, and $615 \mathrm{~nm}$, respectively) (Fig. 16g). All the fabricated devices showed sufficiently high EQEs (22.0 31.8\%) owing to the spontaneous horizontal orientation of molecules (Fig. 16h). Despite the broader bandwidth of EL spectra compared to that of red- and blue-emitting $B B C z-R$ and $B B C z-D B$ devices, BBCz-G and $B B C z-Y$ showed less EQE roll-off owing to their relatively larger ICT character and faster RISC.

Inspired by the $\mathrm{MR}$ effects of $\mathrm{B} / \mathrm{N}$ structures, other structures with different electron-withdrawing groups replacing boron atoms have been reported. For instance, Jiang et al. reported quinolino[3,2,1-de]acridine-5,9-dione (QAO, 5-39), which is composed of two carbonyl groups and one nitrogen atom fused together and exhibits a similar resonance effect ${ }^{110}$. QAO exhibited narrow PL emission at $466 \mathrm{~nm}$ with a FWHM of $32 \mathrm{~nm}$ in toluene; this emission is attributed to the rigid framework with well-separated HOMO and LUMO distributions. The EL spectrum of the QAO-based device was similar to its PL emission spectrum, showing an emission maximum at $468 \mathrm{~nm}$ with an FWHM of $39 \mathrm{~nm}$ and CIE coordinates of $(0.13,0.18)$, demonstrating another type of MR system without boron. Two new types of MR-TADF molecules, 3phenylquinolino[3,2,1-de] acridine-5,9-dione (3-PhQAD, 5-40) and 7-phenylquinolino[3,2,1-de]acridine-5,9-dione (7-PhQAD, 5-41), were developed based on the QAO framework $^{111}$. 3-PhQAD and 7-PhQAD exhibited narrowband emissions at $466 \mathrm{~nm}$ and $464 \mathrm{~nm}$, respectively, with small FWHM values $(<30 \mathrm{~nm})$. Asymmetric 3PhQAD showed a higher EQE (19.1\%) and maximum current efficiency $\left(33.5 \mathrm{~cd} \mathrm{~A}^{-1}\right)$ than 7-PhQAD (EQE of $18.7 \%$ and current efficiency of $28.8 \mathrm{~cd} \mathrm{~A}^{-1}$ ), and both devices showed severe efficiency roll-off due to the loss of excitons via triplet-triplet annihilation (TTA) at low driving voltages and singlet-polaron annihilation (SPA) at higher driving voltages. Colman et al. also modified the QAO structure to synthesize 3,7,11-trimesitylquinolino [3,2,1-de] acridine-5,9-dione $\left(\mathrm{Mes}_{3} \mathrm{DiKTa}, \quad 5-42\right)$ with three orthogonal mesityl groups that reduced intermolecular aggregation and emission quenching ${ }^{112}$. The PL maximum of $\mathrm{Mes}_{3} \mathrm{DiKTa}$ in toluene was $468 \mathrm{~nm}$ with a small FWHM $(27 \mathrm{~nm})$, and redshifted emission at $480 \mathrm{~nm}$ (FWHM $=36 \mathrm{~nm}$ ) was obtained in the film. Although the $\mathrm{Mes}_{3} \mathrm{DiKTa}$-based device showed a higher EQE $(21.1 \%$ at $25 \mathrm{~cd} \mathrm{~m}^{-2}$ ) than the QAO-based device, it also suffered from severe efficiency roll-off. Colman's group also reported dimeric QAO, [3,3'-biquinolino[3,2,1-de]acridine]-5, $5^{\prime}, 9,9^{\prime}$-tetraone (DDiKTa, 5-43), which showed PL emission at $\lambda_{\mathrm{PL}}=470 \mathrm{~nm}$ (FWHM of $47 \mathrm{~nm}$ ) in toluene ${ }^{113}$. Among the devices fabricated with different DDiKTa dopant concentrations (3-12 wt\%) in DPEPO, the $9 \mathrm{wt} \%$ doped device achieved the highest EQE (19\%) with green emission at $500 \mathrm{~nm}$ and CIE coordinates of $(0.18,0.53)$.

$\mathrm{Ma}$ et al. also reported a series of MR-TADF-based QAO derivatives of QA-PF (5-44), QA-PCN (5-45), QA$\mathrm{PMO}(5-46)$, and QA-PCz (5-47) ${ }^{114}$. A design strategy to yield narrow emission was proposed by enhancing the low-frequency vibronic coupling strength while simultaneously reducing the high-frequency vibronic coupling strength of the commonly involved stretching modes. The fluorophenyl groups in QA-PF suppressed the highfrequency stretching vibrations coupled to the structural reorganization between $S_{0}$ and $S_{1}$, resulting in a decrease in the overall reorganization energy. Based on this approach, all the QAO-based derivatives showed a narrower PL emission in toluene $(\mathrm{FWHM}=23-29 \mathrm{~nm})$ compared to that of QAO. The EL devices also showed narrow spectra (FWHM $=27-30 \mathrm{~nm}$ ); however, the EQEs were still lower than those of the $\mathrm{B} / \mathrm{N}$ core structures and suffered from severe efficiency roll-off originating from TTA and SPA. The close intermolecular packing was suggested as one reason for the EQE roll-off.

\section{Conventional D-A type TADF emitters}

Narrow-emissive TADF materials adopting a conventional TADF structure with spatially separated donoracceptor substituents have also been studied (Fig. 17). Lee et al. designed and synthesized 4,4,6,6-tetra( $9 \mathrm{H}$-carbazol9-yl)-[1,1-biphenyl]-3,3-dicarbonitrile (CzBPCN, 5-48) as a narrowband TADF emitter without the MR effect ${ }^{115}$. An isomeric structure of $\mathrm{CzBPCN}, 4,4,5,5$-tetra $(9 \mathrm{H}$-carbazol9-yl)-[1,1-biphenyl]-2,2-dicarbonitrile (CNBPCz), was also synthesized for comparison. Designed by differentiating the position of the donor (carbazole) and acceptor (nitrile) on the biphenyl central core, interlocked donor groups of CzBPCN restricted its molecular motion, resulting in narrow emission at $453 \mathrm{~nm}$ (FWHM of $51 \mathrm{~nm}$ ) in toluene, while $\mathrm{CNBPCz}$, which does not exhibit this interlocking effect, had an FWHM of over $70 \mathrm{~nm}$ at $\lambda_{\mathrm{PL}}=458 \mathrm{~nm}$. In addition, the rigid interlocked architecture of $\mathrm{CzBPCN}$ resulted in a higher PLQY of $94 \%$ compared to that of 


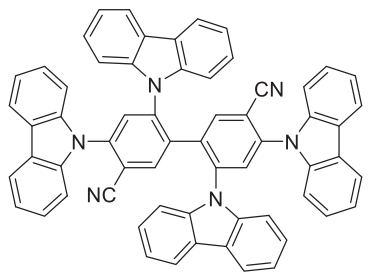

CzBPCN (5-48)

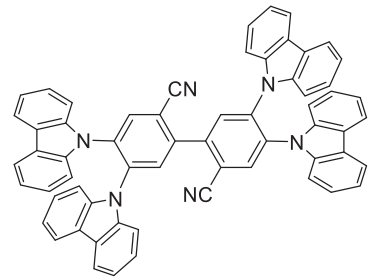

CNBPCZ

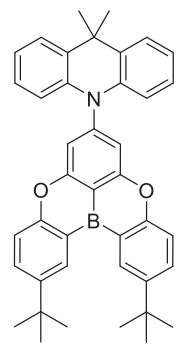

TDBA-AC (5-49)
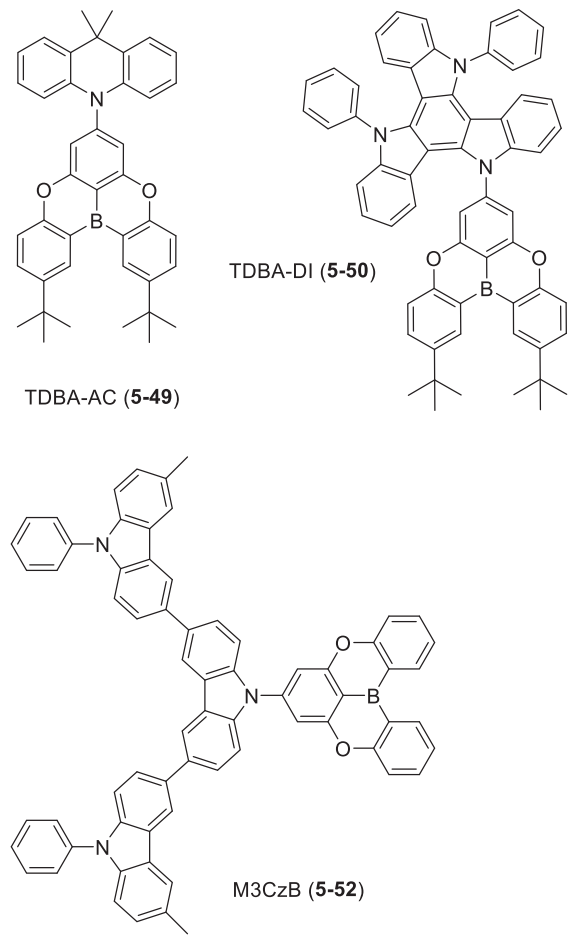

Fig. 17 Chemical structures of conventional D-A-type TADF emitters.

$\mathrm{CNBPCz}$ (37\%). The OLED device fabricated with CzBPCN showed deep blue EL emission at $460 \mathrm{~nm}$ with $\mathrm{FWHM}=48 \mathrm{~nm}$ and CIE coordinates of $(0.14,0.12)$, demonstrating the successful operation of narrowemissive TADF OLEDs without an MR effect. Based on the 5-1 structure as an acceptor, Kwon's group designed two new TADF molecules, 10-(2,12-di-tert-butyl-5,9dioxa-13b-boranaphtho[3,2,1-de] anthracen-7-yl)-9,9dimethyl-9,10-dihydroacridine (TDBA-AC) (5-49) and 5-(2,12-di-tert-butyl-5,9-dioxa-13b-boranaphtho[3,2,1de]anthracen-7-yl)-10,15-diphenyl-10,15-dihydro-5Hdiindolo[3,2-a:3,2-c] carbazole (TDBA-DI) (5-50), which incorporate dimethylacridine and diindolocarbazole as a donating group ${ }^{116}$. The mutually orthogonal conformation between the donating and accepting moieties reduced $\Delta \mathrm{E}_{\mathrm{ST}}$, showing enhanced PLQYs of 93 and $99 \%$ in the DBFPO film. The strong donating ability of the diindolocarbazole in TDBA-DI resulted in a high PLQY (99\%), but its stronger ICT interaction also increased the bandwidth (FWHM of $55 \mathrm{~nm}$ in toluene) compared to that of TDBA-AC (50 nm). OLEDs fabricated with TADF emitters doped in electron-transporting DBFPO (ITO/ HATCN/TAPC/DCDPA/DBFPO:20 wt\% TDBA-AC or TDBA-DI/DBFPO/TPBi/LiF/Al) showed high EQEs for both emitters (25.7\% and $38.15 \%$, respectively) via efficient RISC owing to the mutually orthogonal conformation of the donating and accepting moieties in their structures. Kwon also developed two blue-emitting
TADF molecules, 9'-(2,12-di-tert-butyl-5,9-dioxa-13bboranaphtho[3,2,1-de]anthracen-7-yl)-9,9"-diphenyl$9 H, 9^{\prime} H, 9^{\prime \prime} H-3,3^{\prime}: 6^{\prime}, 3^{\prime \prime}$-tercarbazole (3CzTB, 5-51) and 9'-(5,9-dioxa-13b-boranaphtho[3,2,1-de]anthracen-7-yl)6,6"-dimethyl-9,9"-diphenyl-9H,9' $H, 9^{\prime \prime} H-3,3^{\prime}: 6^{\prime}, 3^{\prime \prime}$-tercarbazole (M3CzB, 5-52), with diphenyltercarbazoles as donating groups ${ }^{117}$. Modification of the alkyl substituent structure and position allows the CT character to be fine controlled. The addition of tert-butyl substituents at the 2 and 12 positions of the $\mathrm{B} / \mathrm{O}$ framework (3CzTB) or methyl substituents of a donor moiety $(\mathrm{M} 3 \mathrm{CzB})$ yielded narrowband emission at 433 and $445 \mathrm{~nm}$ (FWHM $\sim 50 \mathrm{~nm}$ ) in toluene, respectively. Blue OLEDs fabricated with 3CzTB and M3CzB showed high EQEs (29-30\%) and long operational lifetimes of over $81 \mathrm{~h}$ with a slightly larger FWHM (56 and $54 \mathrm{~nm}$, respectively) compared to the MR-TADF emitters.

\section{Summary and outlook}

In this review, fluorescent, phosphorescent, and TADF emitters with narrowband emission were summarized, and the structure-property relationships were discussed. The development of narrow-emission OLED materials is of great importance to meet the new BT 2020 color standard for wide-gamut displays ${ }^{118,119}$. Recently, molecular design strategies and the underlying mechanisms for controlling the emission bandwidth have been studied extensively to achieve narrow PL and EL. 
$\pi$-Conjugated organic fluorophores normally suffer from limited color purity with broad emission owing to their intrinsic vibronic coupling between the $S_{0}$ and $S_{1}$ states as well as structural relaxation in the excited state. Twisted or rigid structures with fused aromatic backbones have shown narrow emissions with a dominant $0-0$ transition caused by inhibition of CT interactions and intermolecular aggregation in the solid state. For example, a narrow EL spectrum (FWHM of $35 \mathrm{~nm}$ ) was observed in TPA-PIM (1-9), which has suppressed vibrational splitting due to its fully twisted donor-acceptor structure, achieving the smallest $\mathrm{CIE}_{\mathrm{y}}$ of 0.046 ever reported at that time $^{31}$. The rigid planar flavanthrone-based dyes (compounds 1-15 17) showed extremely small FWHM values of $\sim 22 \mathrm{~nm}$ at $535 \mathrm{~nm}$ in their EL spectra ${ }^{36}$. The quinacridone or BODIPY-based structures also exhibited a small FWHM $\leq \sim 50 \mathrm{~nm}$ in the green to red region.

The emitting $T_{1}$ state in cyclometalated phosphorescent emitters is often determined by intermixing the ${ }^{3} \mathrm{MLCT}$ and ${ }^{3} \mathrm{LC}$ states. Ir-based octahedral complexes have short phosphorescence lifetimes with high PLQYs due to strong SOC, but their MLCT-dominant emissions result in broad spectra. Several square planar Pt- and Pd-based structures have shown narrow phosphorescence, where the $T_{1}$ state is mainly determined by the ${ }^{3} \mathrm{LC}$ state. By modifying the ligand structures in the cyclometalated $\mathrm{Pt} / \mathrm{Pd}$ complexes, the emission bandwidth can be further decreased, increasing the color purity. $\mathrm{Li}$ et al. modified the rigid tetradentate pyridyl ring of carbazolyl pyridine ancillary ligands to further suppress the vibrational coupling, resulting in improved phosphorescence quantum yield and achieving an FWHM of $<20 \mathrm{~nm}$ with $>80 \%$ PLQY (compounds 3-1 5). In particular, the PtON7-dtb (3-5)based EL device exhibited a high EQE of $24.8 \%$ with dominant emission at $451 \mathrm{~nm}$ (FWHM of $29 \mathrm{~nm}$ ).

TADF molecules can achieve 100\% IQE through efficient upconversion via the RISC process; however, these emitters are often designed by spatial separation of the HOMO and LUMO distributions to decrease the $\Delta \mathrm{E}_{\mathrm{ST}}$ between the $S_{1}$ and $T_{1}$ states. This strategy enhances the structural relaxation in the excited states via ICT, resulting in a broad CT emission with FWHM values of 70-100 nm and large Stokes shifts. Recently, new MRbased TADF molecules with rigid skeletons and regular arrangements of boron and nitrogen atoms have been investigated extensively by Hatakeyama et al. Owing to the MR effects between the electron-accepting boron and electron-donating nitrogen atoms, the strategic separation of the HOMO and LUMO localized around the individual atoms could achieve not only high EQE but also narrow emission bandwidth. For example, MR-TADF OLEDs based on v-DABNA (5-14) demonstrated extremely narrow emission with an FWHM of $18 \mathrm{~nm}$ and a remarkably high EQE of $31.4 \%{ }^{95}$.
Although many promising results for the development of singlet and triplet emitters with narrow emission have been reported, several challenges remain. To further decrease the emission bandwidth, the $0-0$ vibronic transition should be intensified with higher $0-\mathrm{n}(n=1,2,3 \ldots)$ vibronic transitions suppressed. Thus far, it is not clearly understood how the $0-n$ vibronic transitions can be efficiently suppressed or controlled by the molecular design of fluorophores. The vibrational normal modes coupled with the electronic $S_{1}$ to $S_{0}$ transition need to be studied to understand how to control their Huang-Rhys factors and minimize the $0-n$ vibronic transition. The number of green- and red-emitting singlet fluorescent emitters is very limited, with most of the reported narrowband fluorophores being blue-emitting materials. Molecules are often designed to exploit the ICT interaction to extend the effective conjugation with a reduced bandgap; however, the CT character induces undesirable broad emission. Given these limitations, the development of efficient green- and red-emitting fluorescent materials with small FWHM values is of paramount importance. Hyperfluorescent OLEDs made by combining TADF sensitizers and narrow-emitting fluorescent dopants can be an effective strategy to achieve both high EQE and color purity. In the case of phosphorescent triplet emitters, the structural optimization of square planar organometal complexes is still needed. For instance, rigid tetradentate ligands without intraligand or interligand CT interactions can reduce vibronic coupling with decreased emission bandwidth. Similar to the case of fluorescent materials, the investigation of the vibrational normal modes coupled with the LC $S_{1}$ to $S_{0}$ transition to decrease their Huang-Rhys factors may suggest an affordable solution to intensify the $0-0$ transition with reduced $0-n$ vibronic peaks. The compound library of phosphorescent emitters showing narrow emission and high PLQY needs to be further expanded. As shown in Fig. 1d, blue- and greenMR-TADF emitters have been widely studied to achieve narrow emission and high EQE. However, the development of red-emitting MR-TADF molecules is still far behind, and extending the conjugation of these molecules without broadening the emission bandwidth remains challenging. In addition, the efficiency roll-off in MR-TADF OLEDs that occurs at a high current density remains a significant barrier to the industrialization of MR-TADF OLEDs. To overcome the EQE and its roll-off that originates from TTA, SPA, aggregation quenching, etc., the optimization of emitter structures and the optimization of the device architecture should be considered together. For instance, Adachi recently reported an MR-TADF OLED employing $v$-DABNA as an emitter by combining a TADF sensitizer, showing a maximum EQE of $41 \%$ with suppressed efficiency roll-off ${ }^{120}$. Monkman also adopted v-DABNA as a hyperfluorescent emitter, suppressing efficiency roll-off by decreasing the 
excimer quenching with optimization of its doping ratio ${ }^{121}$. In addition, the enhancement of outcoupling efficiency by controlling the molecular orientation of MR-TADF emitters needs to be studied to further improve the EQE and roll-off. As discussed, the underlying mechanism needs to be further clarified, and fine optimization of the host, TADF dopants, and charge injection/transport layers is necessary to improve the charge balance and efficiency roll-off. In particular, special attention should be given to the design of narrowband green emitters because their CIE coordinates need to be further optimized to a further degree than those of blue and red emitters to satisfy the BT 2020 standards. Despite the current challenges, narrowband emissive OLEDs have great potential to become an efficient and extensively adopted display technology in the next generation of highresolution and wide-color gamut displays if both the resolution and color chromaticity are considered.

\section{Acknowledgements}

This work was supported by the National Research Foundation (NRF) of Korea (Grants NRF-2019R1A2C2085290, 2019R1A6A1A11044070,

2016M1A2A2940911, NRF2020M3H4A3081814). This work was supported by the Korea University Grant and LG Display.

\section{Competing interests}

The authors declare no competing interests.

\section{Publisher's note}

Springer Nature remains neutral with regard to jurisdictional claims in published maps and institutional affiliations.

Received: 23 December 2020 Revised: 19 May 2021 Accepted: 1 June 2021. Published online: 16 July 2021

\section{References}

1. Tang, C. W. \& Vanslyke, S. A. Organic electroluminescent diodes. Appl Phys. Lett. 51, 913-915 (1987).

2. Shirota, Y. \& Kageyama, H. Charge carrier transporting molecular materials and their applications in devices. Chem. Rev. 107, 953-1010 (2007).

3. Hong, G. et al. A brief history of OLEDs-emitter development and industry milestones. Adv. Mater. 33, 2005630 (2021).

4. Broadbent, A. D. A critical review of the development of the CIE1931 RGB color-matching functions. Color Res. App/ 29, 267-272 (2004).

5. International Telecommunication Union (ITU), BT.2020. Parameter Values for Ultra-High Definition Television Systems for Production and International Programme Exchange. https://wnw.itu.int/rec/R-REC-BT.2020-1-201406-S/en (2014).

6. Ye, Y., He, Y. W. \& Xiu, X. Y. Manipulating ultra-high definition video traffic. IEEE Multimed. 22, 73-81 (2015).

7. Qian, G. et al. Band gap tunable, donor-acceptor-donor charge-transfer heteroquinoid-based chromophores: near infrared photoluminescence and electroluminescence. Chem. Mater. 20, 6208-6216 (2008).

8. Wang, S. P. et al. Highly efficient near-infrared delayed fluorescence organic light emitting diodes using a phenanthrene-based charge-transfer compound. Angew. Chem. Int Ed. 54, 13068-13072 (2015).

9. Gierschner, J., Mack, H. G., Luer, L. \& Oelkrug, D. Fluorescence and absorption spectra of oligophenylenevinylenes: vibronic coupling, band shapes, and solvatochromism. J. Chem. Phys. 116, 8596-8609 (2002).

10. Zhang, B., Wu, H. Z., Wang, Z. M., Qin, A. J. \& Tang, B. Z. Planarized intramolecular charge transfer on triphenylamine-modified pyrazine and its application in organic light-emitting diodes. J. Mater. Chem. C 8, 4754-4762 (2020).
11. Liu, B. Q. et al. Monocationic iridium(III) complexes with far-red charge-transfer absorption and near-ir emission: synthesis, photophysics, and reverse saturable absorption. Eur. J. Inorg. Chem. 2019, 2208-2215 (2019).

12. Yang, Z. W. et al. Recent advances in quantum dot-based light-emitting devices: challenges and possible solutions. Mater. Today 24, 69-93 (2019).

13. Steckel, J. S., Ho, J. \& Coe-Sullivan, S. QDs generate light for next-generation. Disp. Photonic Spectra 48, 55-61 (2014).

14. Lee, E. et al. "Greener" quantum-dot enabled LCDs with BT.2020 color gamut. SID 41-1, 549-551 (2016).

15. Cho, Y., Choi, Y. K. \& Sohn, S. H. Optical properties of neodymium-containing polymethylmethacrylate films for the organic light emitting diode color filter. Appl. Phys. Lett. 89, 051102 (2006).

16. Frobel, M. et al. Three-terminal RGB full-color OLED pixels for ultrahigh density displays. Sci. Rep. 8, 9684 (2018).

17. Lee, K. T., Han, S. Y., Li, Z. J., Baac, H. W. \& Park, H. J. Flexible high-color-purity structural color filters based on a higher-order optical resonance suppression. Sci. Rep. 9, 14917 (2019).

18. de Jong, M., Seijo, L., Meijerink, A. \& Rabouw, F. T. Resolving the ambiguity in the relation between Stokes shift and Huang-Rhys parameter. Phys. Chem. Chem. Phys. 17, 16959-16969 (2015).

19. Li, K. et al. Highly phosphorescent platinum(II) emitters: photophysics, materials and biological applications. Chem. Sci. 7, 1653-1673 (2016).

20. Li, W. J. et al. A hybridized local and charge-transfer excited state for highly efficient fluorescent OLEDs: molecular design, spectral character, and full exciton utilization. Adv. Opt. Mater. 2, 892-901 (2014).

21. Lin, J. Y. et al. Ultrastable supramolecular self-encapsulated wide-bandgap conjugated polymers for large-area and flexible electroluminescent devices. Adv. Mater. 31, 1804811 (2019).

22. Kim, Y. H. et al. Novel blue emitting material with high color purity. Adv. Mater. 13, 1690-1693 (2001).

23. Park, H. et al. Highly rigid and twisted anthracene derivatives: a strategy for deep blue OLED materials with theoretical limit efficiency. J. Mater. Chem. 22, 2695-2700 (2012).

24. Odom, S. A., Parkin, S. R. \& Anthony, J. E. Tetracene derivatives as potential red emitters for organic LEDs. Org. Lett. 5, 4245-4248 (2003).

25. Park, Y. et al. New blue-violet emitters based on an indenopyrazine core for OLEDs: effects of the position of m-terphenyl side group substitution on optical and electroluminescence properties. Org. Electron 11, 864-871 (2010).

26. Park, Y. I. et al. Synthesis and electroluminescence properties of novel deep blue emitting 6,12-dihydro-diindeno[1,2-b: 1',2'-e] pyrazine derivatives. Chem. Commun. 2008, 2143-2145 (2008).

27. Park, Y. et al. An aromatic imine group enhances the EL efficiency and carrier transport properties of highly efficient blue emitter for OLEDs. J. Mater. Chem. 20, 5930-5936 (2010).

28. Wang, Z. M. et al. Phenanthro[9,10-d]imidazole as a new building block for blue light emitting materials. J. Mater. Chem. 21, 5451-5456 (2011).

29. Gao, Z. et al. High-efficiency violet-light-emitting materials based on phenanthro[9,10-d]imidazole. Chem. Eur. J. 19, 2602-2605 (2013).

30. Gao, Z. et al. Highly efficient deep blue light emitting devices based on triphenylsilane modified phenanthro[9, 10-d]imidazole. Laser Photonics Rev. 8 , L6-L10 (2014).

31. Li, W. J. et al. Highly efficient deep-blue OLED with an extraordinarily narrow FHWM of $35 \mathrm{~nm}$ and a y coordinate $<0.05$ based on a fully twisting donoracceptor molecule. J. Mater. Chem. C 2, 4733-4736 (2014).

32. Jeon, Y. M., Lee, J. Y., Kim, J. W., Lee, C. W. \& Gong, M. S. Deep-blue OLEDs using novel efficient spiro-type dopant materials. Org. Electron 11, 1844-1852 (2010).

33. Cocherel, N., Poriel, C., Vignau, L., Bergamini, J. F. \& Rault-Berthelot, J. Dispiroxanthene-indenofluorene: a new blue emitter for nondoped organic light emitting diode applications. Org. Lett. 12, 452-455 (2010).

34. Jung, $\mathrm{H}$. et al. High efficiency and long lifetime of a fluorescent blue-light emitter made of a pyrene core and optimized side groups. ACS Appl Mater. Interfaces 10, 30022-30028 (2018).

35. Ahn, D. H., Jeong, J. H., Song, J., Lee, J. Y. \& Kwon, J. H. Highly efficient deep blue fluorescent organic light-emitting diodes boosted by thermally activated delayed fluorescence sensitization. ACS Appl Mater. Interfaces 10, 10246-10253 (2018). 
36. Kotwica, K. et al. Soluble flavanthrone derivatives: synthesis, characterization, and application to organic light-emitting diodes. Chem. Eur. J. 22, 7978-7986 (2016).

37. Shah, B. K., Neckers, D. C., Shi, J. M., Forsythe, E. W. \& Morton, D. Anthanthrene derivatives as blue emitting materials for organic light-emitting diode applications. Chem. Mater. 18, 603-608 (2006).

38. Chen, D. J. et al. Efficient solution-processed red all-fluorescent organic lightemitting diodes employing thermally activated delayed fluorescence materials as assistant hosts: molecular design strategy and exciton dynamic analysis. J. Mater. Chem. C 5, 5223-5231 (2017).

39. Debad, J. D., Morris, J. C., Lynch, V., Magnus, P. \& Bard, A. J. Dibenzotetraphenylperiflanthene: synthesis, photophysical properties, and electrogenerated chemiluminescence. J. Am. Chem. Soc. 118, 2374-2379 (1996).

40. Lee, H., Chung, W. J. \& Lee, J. Y. Narrowband and pure violet organic emitter with a full width at half maximum of $14 \mathrm{~nm}$ and gamma color coordinate of below 0.02. Small 16, 1907569 (2020).

41. Ye, K. Q. et al. Supramolecular structures and assembly and luminescent properties of quinacridone derivatives. J. Phys. Chem. B 109, 8008-8016 (2005).

42. $\mathrm{Bi}, \mathrm{H}$. et al. Fluorinated quinacridone derivative based organic light-emitting device with high power efficiency. Org. Electron 11, 1180-1184 (2010).

43. Wang, C. G. et al. Polymorph, assembly, luminescence and semiconductor properties of a quinacridone derivative with extended pi-conjugated framework. J. Mater. Chem. C 1, 5548-5556 (2013).

44. Ivaniuk, K. et al. BODIPY-core 1,7-diphenyl-substituted derivatives for photovoltaics and OLED applications. Dyes Pigm. 175, 108123 (2020).

45. Zatsikha, Y. V., Yakubovskyi, V. P., Shandura, M. P., Dubey, I. Y. \& Kovtun, Y. P. An efficient method of chemical modification of BODIPY core. Tetrahedron 69, 2233-2238 (2013)

46. Bonardi, L. et al. Fine-tuning of yellow or red photo- and electroluminescence of functional difluoro-boradiazaindacene films. Adv. Funct. Mater. 18, 401-413 (2008)

47. Merkushev, D. A. et al. BODIPY associates in organic matrices: Spectral properties, photostability and evaluation as OLED emitters. Mater. Chem. Phys. 187, 104-111 (2017).

48. Santra, M. et al. Dramatic substituent effects on the photoluminescence of boron complexes of 2-(benzothiazol-2-yl)phenols. Chem. Eur. J. 18 9886-9893 (2012).

49. Song, X. Z., Zhang, D. D., Zhang, Y. W., Lu, Y. \& Duan, L. Strategically modulating carriers and excitons for efficient and stable ultrapure-green fluorescent OLEDs with a sterically hindered BODIPY dopant. Adv. Opt. Mater. 8 2000483 (2020).

50. Lamansky, S. et al. Synthesis and characterization of phosphorescent cyclometalated iridium complexes. Inorg. Chem. 40, 1704-1711 (2001).

51. Brooks, J. et al. Synthesis and characterization of phosphorescent cyclometalated platinum complexes. Inorg. Chem. 41, 3055-3066 (2002).

52. Adachi, C., Baldo, M. A., Thompson, M. E. \& Forrest, S. R. Nearly $100 \%$ internal phosphorescence efficiency in an organic light-emitting device. J. Appl. Phys. 90, 5048-5051 (2001)

53. Tsuboyama, A. et al. Homoleptic cyclometalated iridium complexes with highly efficient red phosphorescence and application to organic lightemitting diode. J. Am. Chem. Soc. 125, 12971-12979 (2003).

54. Yersin, H. Highly Efficient OLEDs with Phosphorescent Materials (Wiley-VCH, Weinheim, Germany, 2007).

55. Howarth, A. J., Majewski, M. B. \& Wolf, M. O. Photophysical properties and applications of coordination complexes incorporating pyrene. Coord. Chem. Rev. 282, 139-149 (2015).

56. Heine, J. \& Muller-Buschbaum, K. Engineering metal-based luminescence in coordination polymers and metal-organic frameworks. Chem. Soc. Rev. 42, 9232-9242 (2013).

57. Rausch, A., Homeier, H., Djurovich, P., Thompson, M. \& Yersin, H. Spin-orbit coupling routes and OLED performance: studies of blue-light emitting Ir(III) and Pt (II) complexes. In Proc. SPIE 6655, Organic Light Emitting Materials and Devices XI 66550F (2007).

58. Baldo, M. A., Lamansky, S., Burrows, P. E., Thompson, M. E. \& Forrest, S. R. Very high-efficiency green organic light-emitting devices based on electrophosphorescence. Appl. Phys. Lett. 75, 4-6 (1999).

59. Hofbeck, T. \& Yersin, H. The triplet state of fac-Ir(ppy)(3). Inorg. Chem. 49, 9290-9299 (2010).

60. Kim, H. U. et al. Substituents engineered deep-red to near-infrared phosphorescence from tris-heteroleptic iridium(III) complexes for solution processable red-NIR organic light-emitting diodes. J. Mater. Chem. C 6 10640-10658 (2018).

61. Kim, H. U. et al. Ancillary ligand-assisted robust deep-red emission in iridium (iii) complexes for solution-processable phosphorescent OLEDs. J. Mater. Chem. C 7, 4143-4154 (2019).

62. Ren, B. Y. et al. A yellow-emitting homoleptic iridium(III) complex constructed from a multifunctional spiro ligand for highly efficient phosphorescent organic light-emitting diodes. Inorg. Chem. 56, 8397-8407 (2017).

63. Hohenleutner, A. et al. Rapid combinatorial synthesis and chromatography based screening of phosphorescent iridium complexes for solution processing. Adv. Funct. Mater. 22, 3406-3413 (2012).

64. Jang, J. H. et al. Red phosphorescent naphthalene-based iridium(III) complex for solution-processed single-emissive-layer white organic light-emitting diodes. J. Nanosci. Nanotechnol. 16, 8580-8584 (2016).

65. Fleetham, T., Li, G. J., Wen, L. L. \& Li, J. Efficient "pure" blue OLEDs employing tetradentate Pt complexes with a narrow spectral bandwidth. Adv. Mater. 26, 7116-7121 (2014).

66. Li, G. J., Wolfe, A., Brooks, J., Zhu, Z. Q. \& Li, J. Modifying emission spectral bandwidth of phosphorescent platinum(II) complexes through synthetic control. Inorg. Chem. 56, 8244-8256 (2017).

67. Li J., Turner E. \& Huang L. Metal compounds and methods and uses thereof. USA patent WO 2014031977 A1 (2014).

68. Li, G. J., Fleetham, T., Turner, E., Hang, X. C. \& Li, J. Highly efficient and stable narrow-band phosphorescent emitters for OLED applications. Adv. Opt Mater. 3, 390-397 (2015)

69. Ma, H. L. et al. High-color-purity and efficient solution-processable blue phosphorescent light-emitting diodes with Pt(ii) complexes featuring 3pi pi* transitions. Mater. Chem. Front. 3, 2448-2454 (2019).

70. Baldo, M. A. et al. Highly efficient phosphorescent emission from organic electroluminescent devices. Nature 395, 151-154 (1998).

71. Kwong, R. C. et al. Efficient, saturated red organic light emitting devices based on phosphorescent platinum(II) porphyrins. Chem. Mater. 11 3709-3713 (1999).

72. La Deda, M. et al. Organometallic emitting dyes: palladium(II) nile red complexes. J. Organomet. Chem. 690, 857-861 (2005).

73. Zhu, W. H. \& Fan, L. Q. Room temperature phosphorescence of a palladium (II) complex sensitized by unsymmetric perylene bisimide. Dyes Pigm. 76 663-668 (2008).

74. Zhu, Z. Q., Park, C. D., Klimes, K. \& Li, J. Highly efficient blue OLEDs based on metal-assisted delayed fluorescence Pd(II) complexes. Adv. Opt. Mater. 7 1801518 (2019).

75. Borisov, S. M., Zenkl, G. \& Klimant, I. Phosphorescent platinum(II) and palladium(II) complexes with azatetrabenzoporphyrins-new red laser diodecompatible indicators for optical oxygen sensing. ACS Appl. Mater. Interfaces 2, 366-374 (2010).

76. Niedermair, F. et al. Tunable phosphorescent NIR oxygen indicators based on mixed benzo- and naphthoporphyrin complexes. Inorg. Chem. 49, 9333-9342 (2010).

77. Pang, H. et al. 83-1: Invited paper: optimization of high performance deep red OLEDs using tandem structure for automotive lighting application. SID 51, 1247-1250 (2020).

78. Margulies, E. A. et al. 65-1: Invited paper: narrow spectrum deep red emitters for OLED lighting and display. SID 50, 911-913 (2019).

79. Binnemans, K. Interpretation of europium(III) spectra. Coord. Chem. Rev. 295 1-45 (2015).

80. Binnemans, K. Lanthanide-Based Luminescent hybrid materials. Chem. Rev. 109, 4283-4374 (2009)

81. Hasegawa, Y., Kitagawa, Y. \& Nakanishi, T. Effective photosensitized, electrosensitized, and mechanosensitized luminescence of lanthanide complexes. Npg Asia Mater. 10, 52-70 (2018).

82. Vázquez-lbar, J. L., Weinglass, A. B. \& Kaback, H. R. Engineering a terbium-binding site into an integral membrane protein for luminescence energy transfer. Proc. Natl Acad. Sci. USA 99, 3487-3492 (2002).

83. Li, H. R. et al. Preparation and luminescence properties of hybrid materials containing europium(III) complexes covalently bonded to a silica matrix Chem. Mater. 14, 3651-3655 (2002).

84. Martins, J. P. et al. Highly luminescent pure-red-emitting fluorinated betadiketonate europium(III) complex for full solution-processed OLEDs. J. Lumin 159. 17-25 (2015) 
85. Rajamouli, B., Sood, P., Giri, S., Krishnan, V. \& Sivakumar, V. A dual-characteristic bidentate ligand for a ternary mononuclear europium(III) molecular complex - synthesis, photophysical, electrochemical, and theoretical study. Eur. J. Inorg. Chem. 2016, 3900-3911 (2016).

86. Borisov, S. M., Fischer, R., Saf, R. \& Klimant, I. Exceptional oxygen sensing properties of new blue light-excitable highly luminescent europium(III) and gadolinium(III) complexes. Adv. Funct. Mater. 24, 6548-6560 (2014).

87. Devi, R. \& Vaidyanathan, S. Narrow band red emitting europium complexes and their application in smart white LEDs and vapoluminescent sensors. Dalton Trans. 49, 6205-6219 (2020).

88. $\mathrm{Yu}, \mathrm{G}$. et al. Highly efficient terbium(III)-based organic light-emitting diodes obtained by exciton confinement. J. Mater. Chem. C 4, 121-125 (2016).

89. Chen, Z. Q. et al. A highly efficient OLED based on terbium complexes. Org. Electron 10, 939-947 (2009).

90. IImi, R. et al. A single component white electroluminescent device fabricated from a metallo-organic terbium complex. J. Mater. Chem. C 7, 13966-13975 (2019).

91. Parker, C. A. \& Hatchard, C. G. Triplet-singlet emission in fluid solutions phosphorescence of eosin. Trans. Faraday Soc. 57, 1894-1904 (1961)

92. Endo, A. et al. Efficient up-conversion of triplet excitons into a singlet state and its application for organic light emitting diodes. Appl. Phys. Lett. 98 083302 (2011).

93. Uoyama, H., Goushi, K., Shizu, K., Nomura, H. \& Adachi, C. Highly efficient organic light-emitting diodes from delayed fluorescence. Nature $\mathbf{4 9 2}$ 234-238 (2012).

94. Hatakeyama, T. et al. Ultrapure blue thermally activated delayed fluorescence molecules: efficient HOMO-LUMO separation by the multiple resonance effect. Adv. Mater. 28, 2777-2781 (2016).

95. Kondo, Y. et al. Narrowband deep-blue organic light-emitting diode featuring an organoboron-based emitter. Nat. Photonics 13, 678-682 (2019).

96. Hirai, H. et al. One-step borylation of 1,3-diaryloxybenzenes towards efficient materials for organic light-emitting diodes. Angew. Chem. Int. Ed. $\mathbf{5 4}$ 13581-13585 (2015).

97. Kitamoto, Y. et al. The first synthesis and X-ray crystallographic analysis of an oxygen-bridged planarized triphenylborane. Chem. Commun. 52, 7098-7101 (2016).

98. Nakatsuka, S., Gotoh, H., Kinoshita, K., Yasuda, N. \& Hatakeyama, T. Divergent synthesis of heteroatom-centered 4,8,12-triazatriangulenes. Angew. Chem. Int. Ed. 56, 5087-5090 (2017)

99. Matsui, $K$. et al. One-shot multiple borylation toward BN-doped nanographenes. J. Am. Chem. Soc. 140, 1195-1198 (2018).

100. Oda, S., Kawakami, B., Kawasumi, R., Okita, R. \& Hatakeyama, T. Multiple resonance effect-induced sky-blue thermally activated delayed fluorescence with a narrow emission band. Org. Lett. 21, 9311-9314 (2019).

101. Han, S. H., Jeong, J. H., Yoo, J. W. \& Lee, J. Y. Ideal blue thermally activated delayed fluorescence emission assisted by a thermally activated delayed fluorescence assistant dopant through a fast reverse intersystem crossing mediated cascade energy transfer process. J. Mater. Chem. C 7, 3082-3089 (2019).

102. Liang, $X$. et al. Peripheral amplification of multi-resonance induced thermally activated delayed fluorescence for highly efficient OLEDs. Angew. Chem. Int. Ed. 57, 11316-11320 (2018)

103. Oda, S. et al. Carbazole-based DABNA analogues as highly efficient thermally activated delayed fluorescence materials for narrowband organic lightemitting diodes. Angew. Chem. Int. Ed. 60, 2882-2886 (2021).
104. Xu, Y. C. et al. Molecular-structure and device-configuration optimizations toward highly efficient green electroluminescence with narrowband emission and high color purity. Adv. Opt. Mater. 8, 1902142 (2020).

105. Xu, Y. C. et al. Constructing charge-transfer excited states based on frontier molecular orbital engineering: narrowband green electroluminescence with high color purity and efficiency. Angew. Chem. Int. Ed. 59, 17442-17446 (2020).

106. Zhang, Y. W. et al. Multi-resonance induced thermally activated delayed fluorophores for narrowband green OLEDs. Angew. Chem. Int. Ed. $\mathbf{5 8}$ 16912-16917 (2019).

107. Zhang, Y. W. et al. Achieving pure green electroluminescence with CIEy of 0.69 and EQE of $28.2 \%$ from an aza-fused multi-resonance emitter. Angew. Chem. Int. Ed. 59, 17499-17503 (2020).

108. Ikeda, N. et al. Solution-processable pure green thermally activated delayed fluorescence emitter based on the multiple resonance effect. Adv. Mater. 32, 2004072 (2020).

109. Yang, M. L., Park, I. S. \& Yasuda, T. Full-color, narrowband, and high-efficiency electroluminescence from boron and carbazole embedded polycyclic heteroaromatics. J. Am. Chem. Soc. 142, 19468-19472 (2020).

110. Yuan, Y. et al. The design of fused amine/carbonyl system for efficient thermally activated delayed fluorescence: novel multiple resonance core and electron acceptor. Adv. Opt. Mater. 7, 1801536 (2019).

111. Li, X. et al. Thermally activated delayed fluorescence carbonyl derivatives for organic light-emitting diodes with extremely narrow full width at halfmaximum. ACS Appl. Mater. Interfaces 11, 13472-13480 (2019).

112. Hall, D. et al. Improving processability and efficiency of resonant TADF emitters: a design strategy. Adv. Opt. Mater. 8, 1901627 (2020).

113. Sun, D. M. et al. The design of an extended multiple resonance TADF emitter based on a polycyclic amine/carbonyl system. Mater. Chem. Front. 4 2018-2022 (2020).

114. Qiu, X. et al. Narrowband emission from organic fluorescent emitters with dominant low-frequency vibronic coupling. Adv. Opt. Mater. 9, 2001845 (2021).

115. Cho, Y. J., Jeon, S. K., Lee, S. S., Yu, E. \& Lee, J. Y. Donor interlocked molecular design for fluorescence-like narrow emission in deep blue thermally activated delayed fluorescent emitters. Chem. Mater. 28, 5400-5405 (2016).

116. Ahn, D. H. et al. Highly efficient blue thermally activated delayed fluorescence emitters based on symmetrical and rigid oxygen-bridged boron acceptors. Nat. Photonics 13, 540-546 (2019).

117. Karthik, D. et al. Highly efficient blue thermally activated delayed fluorescence organic light emitting diodes based on tercarbazole donor and boron acceptor dyads. J. Mater. Chem. C 8, 2272-2279 (2020).

118. Masaoka, K., Nishida, Y. \& Sugawara, M. Designing display primaries with currently available light sources for UHDTV wide-gamut system colorimetry. Opt. Express 22, 19069-19077 (2014).

119. Fukagawa, H., Oono, T., Iwasaki, Y., Hatakeyama, T. \& Shimizu, T. Highefficiency ultrapure green organic light-emitting diodes. Mater. Chem. Front. 2, 704-709 (2018).

120. Chan, C. Y. et al. Stable pure-blue hyperfluorescence organic light-emitting diodes with high-efficiency and narrow emission. Nat. Photonics 15, 203-207 (2021).

121. Stavrou, K., Danos, A., Hama, T., Hatakeyama, T. \& Monkman, A. Hot vibrational states in a high-performance multiple resonance emitter and the effect of excimer quenching on organic light-emitting diodes. ACS Appl. Mater. Interfaces 13, 8643-8655 (2021). 